
\title{
Tracing Groundwater Flow Systems with Hydrogeochemistry in Bengal Delta Aquifers, Bangladesh
}

\author{
Ratan Kumar Majumder ${ }^{1 *}$ and Jun Shimada ${ }^{2}$ \\ 'Institute of Nuclear Minerals, Bangladesh Atomic Energy Commission, Dhaka 1349, Bangladesh; \\ ratankm@baec.gov.bd \\ ${ }^{2}$ Graduate School of Science and Technology, Kumamoto University, 2-39-1 Kurokami, Kumamoto 860-8555, Japan; \\ jshimada@sci.kumamoto-u.ac.jp
}

\begin{abstract}
Objective: In present study, hydrochemical data were used to characterize the hydrogeochemical processes and to identify groundwater flow systems in Bengal Delta aquifers, Bangladesh. Methods: Regarding this, 202 shallow, 26 intermediate and 100 deep groundwater samples were collected from the study area for major ion analyses. It is observed that the shallow and intermediate groundwater samples are dominantly $\mathrm{Ca}-\mathrm{Mg}-\mathrm{HCO}_{3}$ type, and the deep groundwater is mainly of $\mathrm{Na}-\mathrm{Cl}-\mathrm{HCO}_{3}$ and $\mathrm{Na}-\mathrm{Cl}$ types. In deep groundwater, the loss of $\mathrm{Ca}^{2+}$ are ion exchanged for $\mathrm{Na}^{+}$along the flow paths, which are initially enriched in $\mathrm{Ca}^{2+}$. The $\mathrm{Na}-\mathrm{HCO}_{3}$ type deep groundwater appear in the coastal confined aquifers, whereas $\mathrm{Na}-\mathrm{Cl}$ type groundwater are found in wells depth ranging from $200-250 \mathrm{~m}$. With some local exceptions, electrical conductivity (EC), $\mathrm{pH}$ and $\mathrm{Cl}^{-}$concentrations for both shallow and deep groundwater gradually increase generally from north-south direction in the study site. Findings: The observed results clearly indicate the presence of three groundwater systems: (i) the shallow groundwater characterized by low ionic concentration; (ii) intermediate groundwater with less evolved ionic chemistry; and (iii) the deep groundwater with higher ionic concentration. Application: This study enables to conceptualize three groundwater flow systems: namely shallow fast circulating fresh young water mixed and moderately mineralized groundwater representing a transition system between the overlying shallow and underlying deep aquifers and the highly mineralized deep groundwater.
\end{abstract}

Keywords: Bangladesh, Bengal Delta, Groundwater flow, Hydrogeochemistry

\section{Introduction}

Hydrogeochemical processes and reactions occurring within groundwater aquifer have a profound effect on groundwater quality. The geochemical properties of groundwater depend on the chemistry of water in the recharge area as well as on different geochemical processes taking place in the subsurface aquifer systems.

The quality of water along the course of its underground movement therefore depends on chemical and physical properties of surrounding rocks, quantitative and qualitative properties of through-flowing water bod- ies, and the products of human activity ${ }^{1}$. During the last two decades, several research groups ${ }^{2,3}$. Studied hydrogeochemistry and groundwater dynamics of the Bengal basin using a variety of techniques. Suggest that the water chemistry of the Ganges-Brahmaputra drainage system is controlled by the presence of carbonates, silicates and sulfides. They also suggest that weathering is dominated by $\mathrm{H}_{2} \mathrm{CO}_{3}$ derived from oxidation of Organic Matter (OM) in the soil and minor $\mathrm{H}_{2} \mathrm{SO}_{4}$ derived from the oxidation of sulfides. Furthermore, Galy and France-Lanord ${ }^{2}$ advocate that $\mathrm{Na}^{+}$and $\mathrm{K}^{+}$are the dominant cations released by the weathering of the alkaline Himalayan silicates because

${ }^{*}$ Author for correspondence 
of lower abundance of Ca-plagioclase in the Himalayas. Magnesium may be introduced from weathering of biotite to form hydrobiotite, vermiculite or smectite ${ }^{4}$. In ${ }^{3}$ suggest that the Ganges-Brahmaputra floodplains have been dominated by carbonate weathering. However, other authors have argued that the development of the foreland basin in front of the Himalayas ${ }^{5}$ has resulted in deposition of silt-dominated sediments in the Ganges-Brahmaputra flood plain, favoring silicate weathering 2 . Most of the recent studies of groundwater chemistry in the Bengal basin have strongly advocate that the redox-related processes in the aquifer are largely controlled by $\mathrm{FeOOH}$ reduction as catalyzed by microbially mediated oxidation of natural $\mathrm{OM}^{6-8}$. The $\mathrm{OM}$ may exist as dissolved organic carbon or peat layers ${ }^{7}$. Carbonic acid produced by OM oxidation reacts with aquifer sediments to produce high concentrations of $\mathrm{HCO}_{3}^{-}$. The groundwater has been found to be anoxic ${ }^{9}$, with frequent detections of sulfide and $\mathrm{CH}_{4}^{7}$ and very little dissolved $\mathrm{O}_{2}$.

Groundwater chemistry so far has been used to infer the groundwater flow systems in the Bengal Delta aquifers. In present study, groundwater chemistry data has evaluated to infer the active hydrogeochemical processes in the Bengal Delta aquifers. Hence, a detailed investigation was carried out to identify the hydrogeochemical process and its relation to groundwater flow system in the Bengal Delta aquifers of Bangladesh.

\section{Study area}

\subsection{Geology, Hydrogeology and Rainfall}

Bangladesh occupies the greater part of the Bengal Delta, which forms largely of alluvial and deltaic sediments of the Ganges-Brahmaputra-Meghna (GBM) rivers system. Excluding the eastern Tertiary Hill Range (Figure 1), the present study covers about $85 \%$ land area of Bangladesh. It is convenient to consider the regional geology in terms of five major subdivisions - Tertiary deposits, Residual deposits, Alluvial fan deposits, Alluvial deposits and Deltaic deposits (Figure 1) $\frac{10,11}{1}$. The residual deposits (the Pleistocene Madhupur and Barind Tracts) locally interrupt the flat topography of central Bangladesh rising by up to $20 \mathrm{~m}$ above the adjacent floodplains $\mathrm{s}^{\underline{11}}$. A generalized geological cross-section (Figure 1) shows the structure of Bengal Basin (Figure 2).

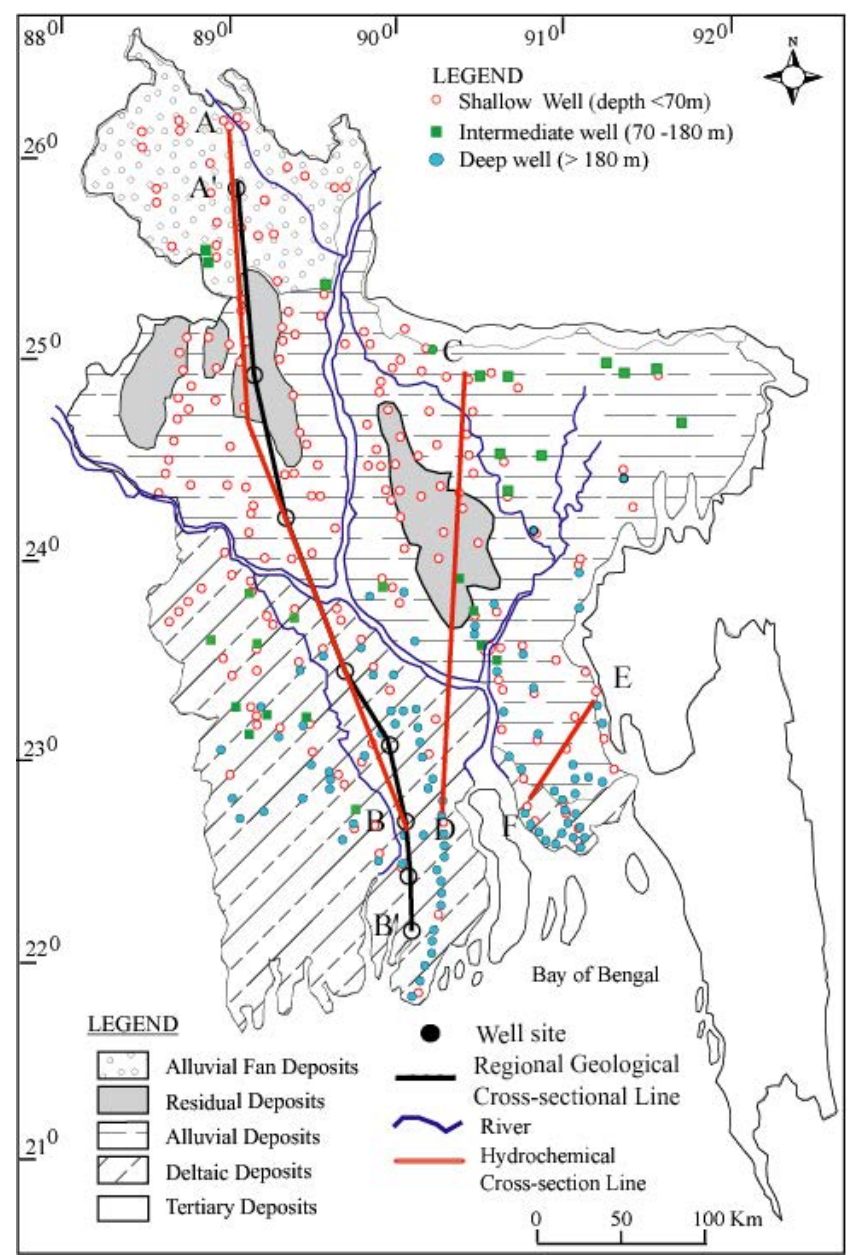

Figure 1. Sample location and surface geological map of Bangladesh (modified after ${ }^{10-12}$ ).

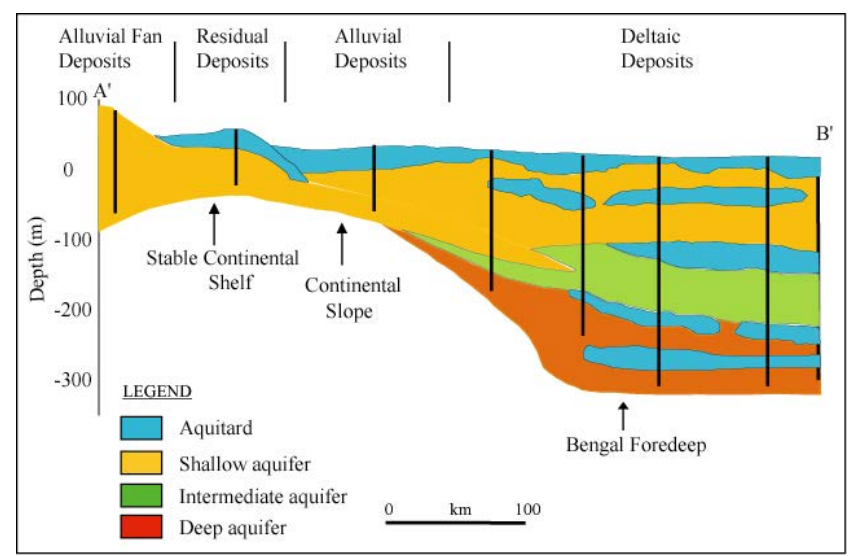

Figure 2. Geological cross-section A'-B' (as shown in Figure 1) through the study area showing borehole locations and the structure of Bengal Basin (modified from ${ }^{13,32}$ ).

The thick unconsolidated deposits of Pleistocene and Holocene alluvial sediments of the GBM delta system 
form one of the most productive aquifer systems in the world ${ }^{12}$. Silts and clays are predominate in the upper few meters of the GBM delta system, forming a surfacial aquitard, generally less than $10 \mathrm{~m}$ thick with typical specific yield values of $2-3 \%$, and vertical permeability values in the range $3-8 \times 10^{-3} \mathrm{~m} / \mathrm{d}$. The aquifers are mostly mediumto-fine and medium-to-coarse sands, with permeability of $40-80 \mathrm{~m} / \mathrm{d}$. Short-term pumping tests on the Holocene aquifers indicate a leaky response, but for longer pumping periods the aquifer is best described as regionally unconfined. The principal mineralogical components of the Holocene sands are quartz, plagioclase feldspars, potassium feldspars, micas (muscovite, biotite and chlorite), and clays (smectite, kaolinite and illite). Deep clayey aquitards exist in coastal regions and the sands below the aquitards are commonly referred to as the deep aquifer. In present study, based on the sampled well depths, the studied aquifers are considered as shallow $(<70 \mathrm{~m})$, intermediate $(70-180 \mathrm{~m})$ and deep $(>180 \mathrm{~m})$ aquifers ${ }^{12}$.

The average annual rainfall in Bangladesh varies from a maximum of $5690 \mathrm{~mm}$ in the northeast of the country to minimum of $1110 \mathrm{~mm}$ in the west. Up to $95 \%$ of the annual rainfall occurs during the May to September monsoon.

\section{Methodology}

\subsection{Groundwater Sampling}

A total of 202 shallow, 26 intermediate and 100 deep groundwater samples were collected during the sampling campaigns (January - February, 2006; November - December, 2006; September - October, 2007 and March 2008). Sampled wells were chosen arbitrarily (Figure 1) and prior to sampling each well were pumped for several minutes until it purged out approximately twice the well volume, or until steady state chemical conditions $(\mathrm{pH}$, electrical conductivity and Temperature) were obtained. The geographical location of each well was determined with a GARMIN handheld global positioning system (Kansas, USA) and the approximate depth of wells were noted from the well owner's records. The physical parameters electrical conductivity, $\mathrm{pH}$ and temperature were measured with a portable $\mathrm{EC} / \mathrm{pH}$ meter (TOA EC/pH METER, WM-22EP). Samples for major ion analysis were collected in $100 \mathrm{~mL}$ High Density Polyethylene (HDPE) bottle. All the samples were stored at a temperature of $4^{\circ} \mathrm{C}$ until analysis.

\subsection{Laboratory Analyses}

The major cations $\left(\mathrm{Ca}^{2+}, \mathrm{Mg}^{2+}, \mathrm{K}^{+}\right.$and $\left.\mathrm{Na}^{+}\right)$and anions $\left(\mathrm{Cl}^{-}, \mathrm{NO}_{3}{ }^{-}\right.$and $\left.\mathrm{SO}_{4}{ }^{2-}\right)$ were analyzed with an ion chromatography (Metrohm 761 Compact IC). The instrument was linearly calibrated from 2.5 to $7.5 \mathrm{mg} / \mathrm{L}$ with standards (Wako Pure Chemicals Industries Ltd., Japan). All of the samples were diluted several times to adjust for the operating range. Alkalinity (as $\mathrm{HCO}_{3}^{-}$) was determined by field titration with $1.6 \mathrm{~N} \mathrm{H}_{2} \mathrm{SO}_{4}$ to $\mathrm{pH} \sim 4.5$ using HACH Digital multi Sampler Model 1690.

The potential for a chemical reaction can be determined by calculating the chemical equilibrium of the water with the mineral phase $\mathrm{i}^{\frac{14}{4}}$. The equilibrium state of water with respect to a mineral phase can be determined by calculating a saturation index (SI). The saturation indices were calculated using PHREEQC ${ }^{15}$ with thermodynamic database of MINTEQA2 ${ }^{16}$ and the calculated SI values for calcite $\left(\mathrm{SI}_{\text {calcite }}\right)$ and dolomite $\left(\mathrm{SI}_{\text {dolomite }}\right)$ are given in Table 1 . The SI is defined as the logarithm of the ratio of ion activity product (IAP) to the mineral equilibrium constant at a given temperature and given as: $\mathrm{SI}=$ $\log _{10}\left(\mathrm{IAP} / \mathrm{K}_{\mathrm{sp}}\right)$, where IAP = ion activity product and $\mathrm{K}_{\mathrm{sp}}$ $=$ solubility product at given temperature ${ }^{14}$.

\section{Results}

\subsection{Physical Parameters}

Physical parameters and major ion concentrations of analyzed water samples are given in Table 1 . The shallow and intermediate depth groundwater show low mineralization with EC ranging from $282-920 \mu \mathrm{S} / \mathrm{cm}$ (average $637 \mu \mathrm{S} / \mathrm{cm}$ ) and $282-547 \mu \mathrm{S} / \mathrm{cm}$ (average $531 \mu \mathrm{S} / \mathrm{cm}$ ) respectively, and the deep groundwater $\mathrm{EC}$ values varied from $117-4870 \mu \mathrm{S} / \mathrm{cm}$ (average $1288 \mu \mathrm{S} / \mathrm{cm}$ ). The shallow groundwater temperature ranges from $23-30.2^{\circ} \mathrm{C}$ with an average value of $26^{\circ} \mathrm{C}$, while the intermediate depth groundwater temperature varies from $22.4-28^{\circ} \mathrm{C}$ (average $25.9^{\circ} \mathrm{C}$ ). However, the deep groundwater average temperature is $27.4^{\circ} \mathrm{C}$ varying from $24.8-29.5^{\circ} \mathrm{C}$. The shallow groundwater $\mathrm{pH}$ values vary from $5.5-7.96$ with an average value of 6.88 . However, the intermediate depth groundwater average $\mathrm{pH}$ value is near neutral (7.07) and the deep groundwater average $\mathrm{pH}$ value (7.4) is higher than that of the shallow and intermediate well groundwater average $\mathrm{pH}$ values. 


\begin{tabular}{|c|c|c|c|c|c|c|c|c|c|c|c|c|c|c|c|c|c|c|c|c|c|c|c|c|c|c|c|c|}
\hline $5^{\frac{5}{8}}$ & & 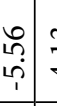 & $\begin{array}{ll}m & \\
+ & i\end{array}$ & $\begin{array}{l}\vec{t} \\
\dot{r}\end{array}$ & $\underset{+}{ \pm}$ & $\begin{array}{c}\infty \\
\stackrel{7}{1} \\
\end{array}$ & 암 & $\stackrel{\text { f }}{\stackrel{1}{1}}$ & $\mid \begin{array}{c}n \\
\tilde{n} \\
i \\
i\end{array}$ & • & 움 & $\begin{array}{c}4 \\
\stackrel{4}{-1} \\
\end{array}$ & $\begin{array}{c}+ \\
\stackrel{+}{0} \\
0\end{array}$ & $\stackrel{+}{0}$ & $\begin{array}{l}n \\
\text { ?n } \\
\vdots \\
\end{array}$ & \begin{tabular}{|c|}
$\infty$ \\
\hdashline \\
0 \\
1 \\
\end{tabular} & $\begin{array}{l}\hat{0} \\
\dot{1} \\
\end{array}$ & 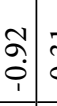 & & & $\begin{array}{l}\vec{N} \\
\stackrel{i}{1} \\
\end{array}$ & $\begin{array}{l}+ \\
\vdots \\
0 \\
0\end{array}$ & $\begin{array}{ll} \\
0 \\
0\end{array}$ & $\begin{array}{l}0 \\
0 \\
0 \\
\end{array}$ & $\mid$\begin{tabular}{c}
0 \\
\hdashline \\
0 \\
\end{tabular} & 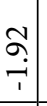 & $\begin{array}{l}8 \\
: \\
\dot{0} \\
\end{array}$ & ্ָ \\
\hline$\omega^{\frac{2}{\frac{5}{5}}}$ & & 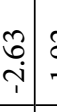 & 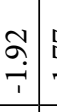 & 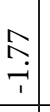 & $\begin{array}{l}\infty \\
\stackrel{1}{i} \\
i\end{array}$ & in & 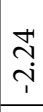 & $\begin{array}{l}R \\
0 \\
i \\
1\end{array}$ & $\mid \begin{array}{l}\infty \\
\rightarrow \\
-1 \\
\end{array}$ & $\begin{array}{l}0 \\
\\
\\
\end{array}$ & 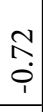 & \begin{tabular}{c}
$\mathfrak{7}$ \\
\hdashline \\
\hdashline \\
\end{tabular} & $\begin{array}{l}0 \\
0 \\
1\end{array}$ & $\begin{array}{l}8 \\
0 \\
0\end{array}$ & $\begin{array}{l}1 \\
\vdots \\
0 \\
0 \\
\end{array}$ & $\begin{array}{l}1 \\
\hat{0} \\
i \\
\end{array}$ & 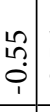 & 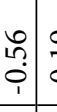 & $\begin{array}{l}2 \\
0 \\
\end{array}$ & $\begin{array}{l}+ \\
\dot{0} \\
\end{array}$ & $\begin{array}{l}4 \\
0 \\
0\end{array}$ & \begin{tabular}{ll}
\multirow{1}{*}{} \\
0 \\
\\
1
\end{tabular} & 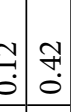 & $=$ & $\begin{array}{c}0 \\
\text { fú } \\
0 \\
\end{array}$ & $\begin{array}{l}0 \\
0 \\
0 \\
1\end{array}$ & 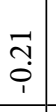 & \\
\hline 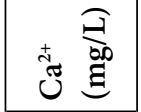 & & 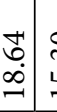 & 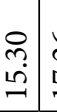 & $\begin{array}{l}\text { ָ̦ } \\
\\
-1\end{array}$ & ?̊. & 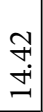 & $\begin{array}{l}7 \\
\infty \\
\infty \\
-\end{array}$ & $\begin{array}{l}n \\
\hat{n} \\
\dot{m} \\
n\end{array}$ & $\begin{array}{l}\infty \\
\infty \\
\pm \\
\dot{+} \\
\end{array}$ & $\begin{array}{l}10 \\
0 \\
0 \\
i\end{array}$ & $\begin{array}{l}\text { ㅇ. } \\
\text { ลे. }\end{array}$ & $\left|\begin{array}{l}2 \\
\hat{2} \\
2 \\
2\end{array}\right|$ & 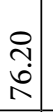 & $\left|\begin{array}{l}\hat{\hat{N}} \\
\hat{n}\end{array}\right|$ & $\begin{array}{l}+1 \\
\dot{0} \\
-\end{array}$ & 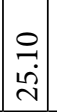 & 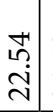 & 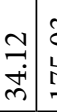 & & $\begin{array}{c}2 \\
m \\
\dot{q} \\
\end{array}$ & $\begin{array}{l}8 \\
: \\
\text { in } \\
\text { in }\end{array}$ & & 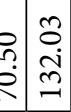 & $\mid \begin{array}{l}\infty \\
\infty \\
0 \\
6\end{array}$ & $\begin{array}{l}\overrightarrow{0} \\
\dot{2} \\
\dot{2}\end{array}$ & $\vec{m}$ & $\begin{array}{l}\hat{b} \\
\text { घa. }\end{array}$ & $\stackrel{+}{\stackrel{ \pm}{二}}$ \\
\hline 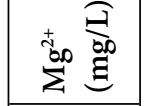 & & 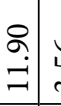 & $\begin{array}{l}n \\
\stackrel{n}{n} \\
\end{array}$ & $\begin{array}{l}+1 \\
\stackrel{+}{+} \\
\end{array}$ & กิ & $\begin{array}{l}0 \\
\dot{0} \\
i\end{array}$ & 余 & $\stackrel{N}{N}$ & $\begin{array}{l}m \\
0 \\
\\
\end{array}$ & in & $\begin{array}{l}\infty \\
\stackrel{2}{+} \\
\dot{ \pm} \\
\end{array}$ & 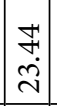 & $\begin{array}{l}\stackrel{10}{二} \\
\pm \\
= \\
=\end{array}$ & $\begin{array}{l}\infty \\
\infty \\
\\
\end{array}$ & $\begin{array}{l}\vec{m} \\
\dot{\sim} \\
\vec{i}\end{array}$ & 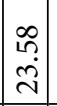 & $\begin{array}{l}2 \\
2 \\
\\
\end{array}$ & & & $\begin{array}{l}\delta \\
\text { in } \\
-1\end{array}$ & 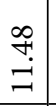 & & 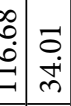 & 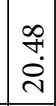 & $\begin{array}{l}= \\
= \\
=\end{array}$ & 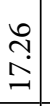 & $\begin{array}{l}\vec{v} \\
\vec{v} \\
\vec{v}\end{array}$ & $\begin{array}{l}\stackrel{8}{\circ} \\
\stackrel{1}{n}\end{array}$ \\
\hline 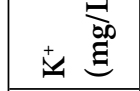 & & $\begin{array}{l}8 \\
\dot{1} \\
\dot{n}\end{array}$ & 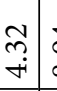 & $\begin{array}{l}+1 \\
0 \\
\infty\end{array}$ & \begin{tabular}{l}
0 \\
\multirow{2}{*}{} \\
in
\end{tabular} & 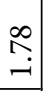 & $\begin{array}{l}\infty \\
\infty \\
- \\
\end{array}$ & 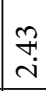 & $\begin{array}{l}3 \\
. \\
- \\
\end{array}$ & $\begin{array}{l}\hat{0} \\
\dot{i n} \\
\text { in }\end{array}$ & $\begin{array}{l}8 \\
\stackrel{1}{0} \\
\end{array}$ & $\begin{array}{c}\text { î } \\
\text { in }\end{array}$ & $\stackrel{-}{n}$ & \begin{tabular}{|l}
$\hat{n}$ \\
in \\
\end{tabular} & . & $\begin{array}{l}2 \\
\alpha \\
\dot{b}\end{array}$ & $\begin{array}{l}2 \\
\stackrel{2}{0} \\
\end{array}$ & 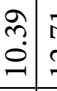 & & $\begin{array}{l}m \\
\infty \\
+ \\
+ \\
\end{array}$ & $\begin{array}{l}\infty \\
\infty \\
i\end{array}$ & 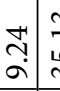 & 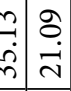 & $\begin{array}{l}\tilde{\omega} \\
\alpha \\
\end{array}$ & 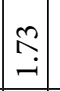 & $\begin{array}{l}\stackrel{n}{n} \\
\\
\end{array}$ & $\begin{array}{l}n \\
1 ? \\
\infty \\
\infty\end{array}$ & $\begin{array}{l}\sim \\
i \\
i\end{array}$ \\
\hline 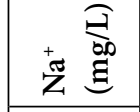 & & $\begin{array}{l}+ \\
\\
\end{array}$ & \begin{tabular}{ll|l}
8 & \\
$\infty$ & & \\
$\infty$ &
\end{tabular} & 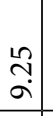 & $\begin{array}{c}n \\
\tilde{m} \\
2 \\
\end{array}$ & 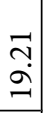 & $\begin{array}{l}0 \\
0 \\
i n \\
-1 \\
\end{array}$ & $\begin{array}{l}\stackrel{m}{2} \\
i \\
i \\
n\end{array}$ & 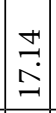 & $\begin{array}{l}0 \\
n \\
i \\
i n\end{array}$ & $\begin{array}{l}\underset{1}{\infty} \\
\text { İ }\end{array}$ & $\left|\begin{array}{l}0 \\
\vdots \\
i \\
\dot{f}\end{array}\right|$ & 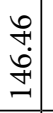 & $\mid \begin{array}{c}\vec{\sim} \\
\vec{\sim}\end{array}$ & 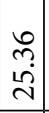 & 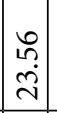 & \begin{tabular}{l}
$\exists$ \\
\multirow{2}{0}{} \\
0
\end{tabular} & 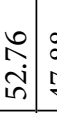 & & $\begin{array}{l}\sigma \\
\sigma \\
\sigma\end{array}$ & 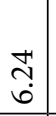 & & 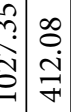 & $\begin{array}{l}\vec{b} \\
\dot{d} \\
\end{array}$ & $\left|\begin{array}{c}\stackrel{a}{\partial} \\
\stackrel{+}{i}\end{array}\right|$ & $\begin{array}{l}\text { î. } \\
\text { in }\end{array}$ & 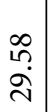 & $\stackrel{m}{6}$ \\
\hline 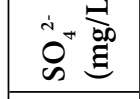 & & $\begin{array}{l}\exists \\
0\end{array}$ & \begin{tabular}{l|l}
$\infty$ & 1 \\
$\infty$ & \\
$\infty$ & $:$ \\
\end{tabular} & $\begin{array}{l}10 \\
m \\
m\end{array}$ & $\begin{array}{l}\stackrel{n}{n} \\
\tilde{n}\end{array}$ & ప. & $\begin{array}{l}\stackrel{+}{\infty} \\
\stackrel{2}{n} \\
\end{array}$ & 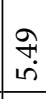 & $\mid \begin{array}{l}m \\
\stackrel{n}{i}\end{array}$ & $\begin{array}{l}3 \\
\\
-\end{array}$ & $\begin{array}{l}\infty \\
0 \\
\infty \\
n \\
\end{array}$ & $\mid \begin{array}{c}N \\
\mathbb{Z} \\
\end{array}$ & $\begin{array}{c}\infty \\
\stackrel{1}{m} \\
\stackrel{m}{n}\end{array}$ & $\begin{array}{l}\stackrel{\pi}{1} \\
0 \\
\end{array}$ & 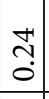 & $\begin{array}{l}\tilde{6} \\
0 \\
\end{array}$ & ָ̊. & 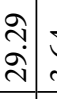 & ț. & 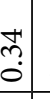 & $\stackrel{n}{n}$ & 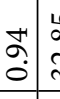 & 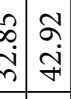 & $\begin{array}{l}\overrightarrow{0} \\
\dot{i} \\
\end{array}$ & $\begin{array}{l}\overrightarrow{0} \\
0 \\
\end{array}$ & 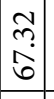 & ஸे & in \\
\hline 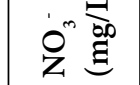 & & $\begin{array}{l}n \\
\\
0\end{array}$ & \begin{tabular}{l|l}
8 & \\
i &
\end{tabular} & $\stackrel{\square}{i}$ & 워 & ?ִ & $\begin{array}{l}\infty \\
\stackrel{\infty}{r} \\
m\end{array}$ & $\begin{array}{l}8 \\
0 \\
0\end{array}$ & $\mid \begin{array}{c}\infty \\
\stackrel{1}{0} \\
0\end{array}$ & $\stackrel{20}{=}$ & 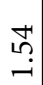 & $\mid$\begin{tabular}{l|}
$\infty$ \\
0 \\
$\dot{m}$
\end{tabular} & $\begin{array}{l}8 \\
\dot{r} \\
\end{array}$ & $\left|\begin{array}{c}1 \\
\infty \\
+ \\
+\end{array}\right|$ & $\left|\begin{array}{l}\infty \\
\infty \\
+\end{array}\right|$ & $\mid$ & $\begin{array}{l}0 \\
\stackrel{1}{-i}\end{array}$ & 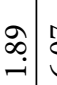 & $\begin{array}{l}\hat{0} \\
\dot{0}\end{array}$ & $\begin{array}{l}\vec{b} \\
\dot{i} \\
\end{array}$ & $\begin{array}{l}\infty \\
\infty \\
0\end{array}$ & $\underset{8}{8}$ & 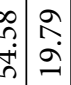 & $\mid \begin{array}{l}\stackrel{m}{+} \\
\dot{0}\end{array}$ & $\mid \begin{array}{c}\hat{N} \\
0\end{array}$ & $\begin{array}{l}7 \\
2 \\
2 \\
2\end{array}$ & $\begin{array}{l}0 \\
\stackrel{-}{\exists} \\
=\end{array}$ & 하 \\
\hline 它 & & 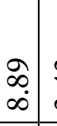 & 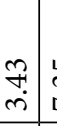 & $\stackrel{m}{n}$ & $\mid \begin{array}{c}\vec{n} \\
\dot{ \pm}\end{array}$ & $\begin{array}{l}\stackrel{+}{N} \\
\stackrel{2}{\sim} \\
\end{array}$ & $\underset{F}{F}$ & f̊. & $\mid \begin{array}{l}\hat{\alpha} \\
\dot{\psi}\end{array}$ & $\begin{array}{l}n \\
\hat{\sigma} \\
\hat{\sigma}\end{array}$ & $\begin{array}{l}\mathbb{N} \\
\infty \\
\infty \\
-1\end{array}$ & $\mid \begin{array}{l}3 \\
6 \\
2 \\
-1\end{array}$ & 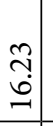 & $\mid \begin{array}{c}n \\
0 \\
\infty \\
-1\end{array}$ & $\stackrel{\infty}{\stackrel{n}{N}}$ & $\mid \begin{array}{l}\infty \\
\infty \\
n \\
\end{array}$ & 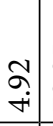 & 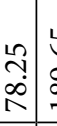 & . & $\begin{array}{l}8 \\
\dot{+} \\
\dot{+}\end{array}$ & $\begin{array}{l}0 \\
\stackrel{0}{i} \\
\end{array}$ & 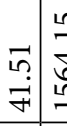 & 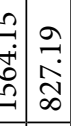 & $\mid \begin{array}{l}+1 \\
1 \\
0 \\
0 \\
m\end{array}$ & $\left|\begin{array}{l}\mathfrak{i} \\
\mathrm{i}\end{array}\right|$ & $\mid \begin{array}{l}-1 \\
0 \\
0 \\
0\end{array}$ & $\begin{array}{c}\hat{i} \\
\text { กิ }\end{array}$ & $\begin{array}{l}\text { oे } \\
\text { ¿ें }\end{array}$ \\
\hline 宅 & & $\vec{n}$ & 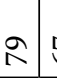 & $\widehat{6}$ & 6 & a & 6 & $\widetilde{\sigma}$ & ๖ & $\stackrel{\stackrel{\leftrightarrow}{\sim}}{\sim}$ & 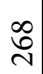 & $\mid$\begin{tabular}{l}
$\infty$ \\
\multirow{2}{*}{}
\end{tabular} & $\begin{array}{l}8 \\
: \\
i\end{array}$ & $\mid \begin{array}{l}1 \\
2 \\
q\end{array}$ & $\overrightarrow{\mathrm{n}}$ & $\left|\begin{array}{l}n \\
2 \\
i \\
n\end{array}\right|$ & $\frac{a}{n}$ & Î̉ & \begin{tabular}{c}
$\infty$ \\
\multirow{2}{*}{} \\
\end{tabular} & : & $\stackrel{\sim}{\sim}$ & $\frac{a}{6}$ & 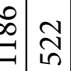 & 角 & $\underset{\widetilde{\sim}}{ }$ & $\mid \begin{array}{l}\infty \\
\infty \\
\infty\end{array}$ & $\stackrel{\circ}{\sim}$ & $\Re$ \\
\hline 仓्ञ & & $\stackrel{+}{\Delta}$ & $\stackrel{2}{2}$ & $\stackrel{ \pm}{I}$ & $\begin{array}{l}\infty \\
\infty \\
\infty\end{array}$ & $\stackrel{n}{\sim}$ & iิ & શิ & $\vec{i}$ & 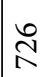 & î & $\underset{\infty}{+}$ & $\begin{array}{l}\mathbb{1} \\
\infty\end{array}$ & $\mid \begin{array}{l}\infty \\
\infty\end{array}$ & $\begin{array}{l}\widetilde{N} \\
\infty\end{array}$ & $\left|\begin{array}{c}\tilde{\infty} \\
\infty\end{array}\right|$ & $\tilde{\infty}$ & 望 & $\vec{\infty}$ & 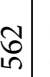 & ì & ‡ి & 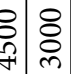 & $\stackrel{尺}{\wedge}$ & $\mid \begin{array}{c}\infty \\
\stackrel{m}{m}\end{array}$ & $\left|\begin{array}{c}0 \\
\infty \\
\infty \\
\infty\end{array}\right|$ & 斈 & $\stackrel{\partial}{\approx}$ \\
\hline 密 ठ & & $\stackrel{2}{2}$ & 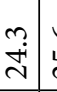 & $\begin{array}{l}0 \\
\dot{i} \\
\dot{i}\end{array}$ & 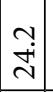 & $\begin{array}{l}10 \\
\text { in } \\
\end{array}$ & $\stackrel{\text { İ }}{\mathrm{i}}$ & กี & $\mid \begin{array}{l}\stackrel{+}{+} \\
\stackrel{+}{N}\end{array}$ & ñ. & $\overrightarrow{0}$ & $\mid \begin{array}{l}\stackrel{+}{d} \\
\text { d }\end{array}$ & ¿̊. & $\mid \begin{array}{c}n \\
\stackrel{0}{0}\end{array}$ & $\begin{array}{l}0 \\
0 \\
\dot{d} \\
\end{array}$ & 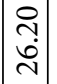 & $\begin{array}{l}\infty \\
\infty \\
\dot{n} \\
\end{array}$ & 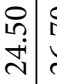 & 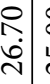 & $\begin{array}{l}8 \\
\text { ì. } \\
\text {. }\end{array}$ & $\begin{array}{l}0 \\
\text { ñ. } \\
\text { ñ }\end{array}$ & 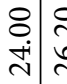 & 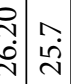 & 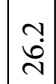 & 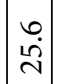 & $\left|\begin{array}{c}0 \\
\dot{i} \\
\text { in }\end{array}\right|$ & $\vec{i}$ & సֶ. \\
\hline 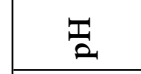 & & $\begin{array}{l}0 \\
0 \\
0 \\
0\end{array}$ & 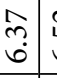 & గึ. & $\begin{array}{l}0 \\
6 \\
0\end{array}$ & కే & $\begin{array}{l}8 \\
0 \\
\end{array}$ & $\begin{array}{l}\Omega \\
\\
\end{array}$ & 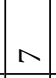 & $\begin{array}{l}4 \\
\text { hn } \\
6 \\
\end{array}$ & $\begin{array}{l}2 \\
0 \\
0 \\
\end{array}$ & \begin{tabular}{|c|}
$n$ \\
6 \\
\end{tabular} & $\begin{array}{l}0 \\
0 \\
0 \\
0\end{array}$ & $\stackrel{n}{N}$ & बे. & $\mid \begin{array}{l}8 \\
0 \\
0\end{array}$ & $\begin{array}{l}1 \\
\infty \\
0 \\
0\end{array}$ & $\begin{array}{l}0 \\
\text { bे } \\
\dot{0}\end{array}$ & 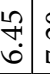 & ָָ & 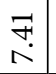 & $\begin{array}{c}\hat{N} \\
\end{array}$ & \begin{tabular}{l|l}
\multirow{2}{*}{} \\
\hdashline \\
\end{tabular} & $\stackrel{2}{\longrightarrow}$ & $\exists$ & $\mid \begin{array}{l}\overrightarrow{0} \\
6 \\
\end{array}$ & $\begin{array}{l}\circ \\
\text { ڤ్ }\end{array}$ & $\stackrel{\infty}{6}$ \\
\hline 卷 & & तี & $\infty:$ & $\simeq$ & $\stackrel{\sharp}{\sim}$ & $m$ & 으 & ‡ & ㄱ. & $\stackrel{-}{-1}$ & $\underset{v}{2}$ & ה & $\hat{\sim}$ & $\stackrel{\sim}{2}$ & $\vec{v}$ & $\infty$ & $\stackrel{2}{\sim}$ & ¿ & 이: & 8 & if & 8 & $\begin{array}{l}n \\
m\end{array}$ & $\simeq$ & กิ & ㄱ. & $\ddot{\sim}$ & $\vec{\sim}$ \\
\hline$\stackrel{\Xi}{\square}$ & 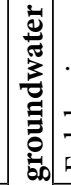 & 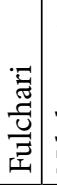 & 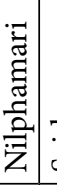 & 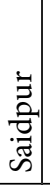 & 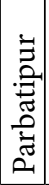 & 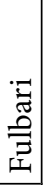 & 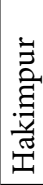 & 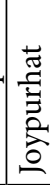 & 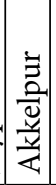 & 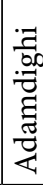 & $\overrightarrow{\bar{\pi}}$ & 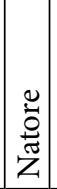 & 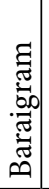 & $\mid$ & 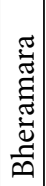 & 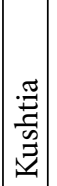 & 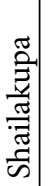 & 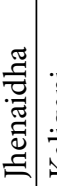 & 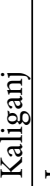 & 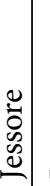 & $\begin{array}{l}\stackrel{0}{0} \\
\mathscr{0} \\
\stackrel{0}{\varrho}\end{array}$ & 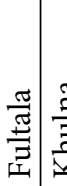 & 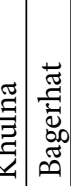 & 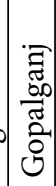 & 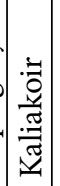 & 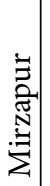 & 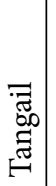 & 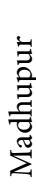 \\
\hline$=$ & 馬 & -1 & 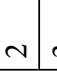 & $m$ & 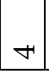 & in & 6 & n & $\infty$ & $a$ & 으 & $\exists$ & $\simeq$ & $\stackrel{m}{-}$ & \pm & 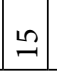 & $\stackrel{0}{-1}$ & $\triangle 9$ & $\infty$ & 2 & $\stackrel{\sim}{1}$ & $\overrightarrow{\mathrm{N}}$ & $\vec{\sim} \tilde{\sim}$ & $\stackrel{\sim}{\sim}$ & $\stackrel{\sim}{\sim}$ & $\stackrel{\sim}{\sim}$ & $\widehat{\curvearrowright}$ & ஓ \\
\hline
\end{tabular}




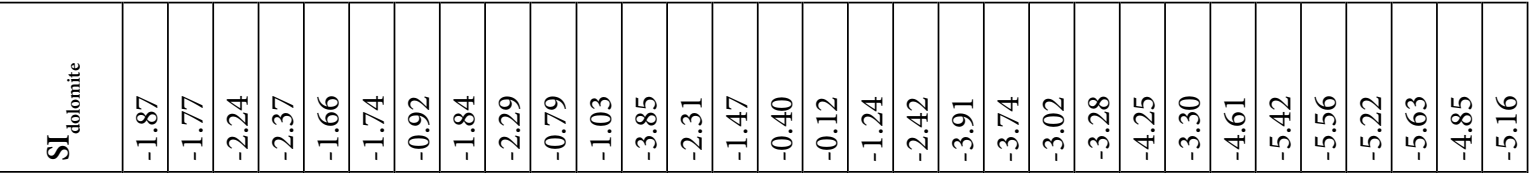

䒕

ש

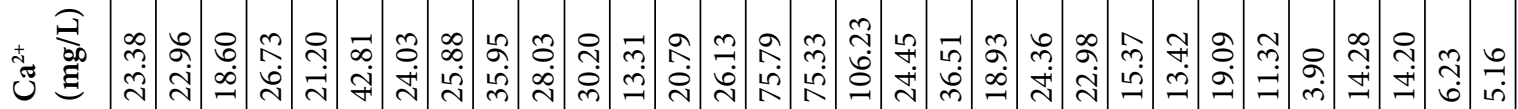

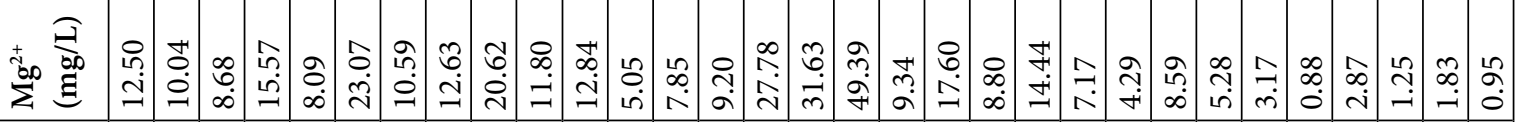

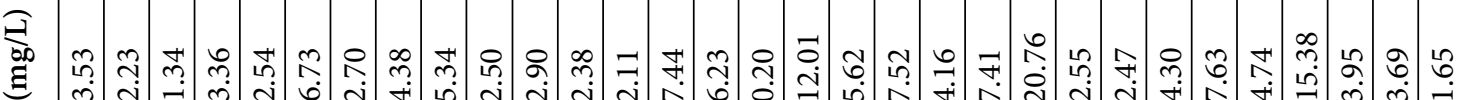

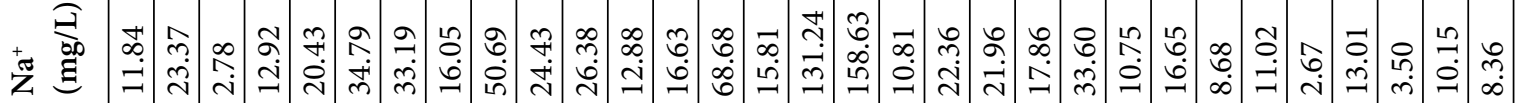

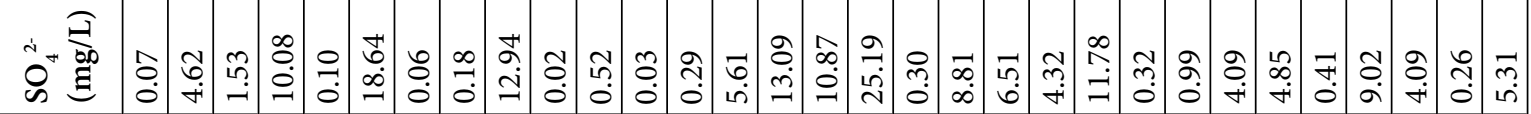

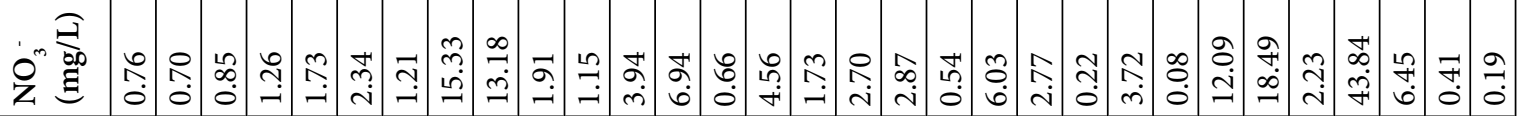

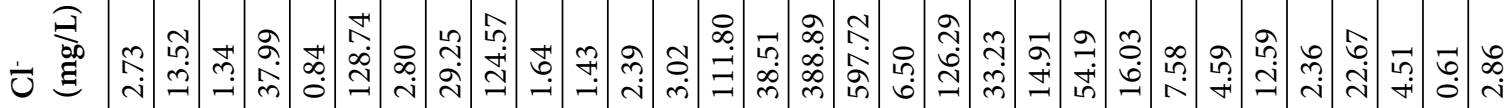

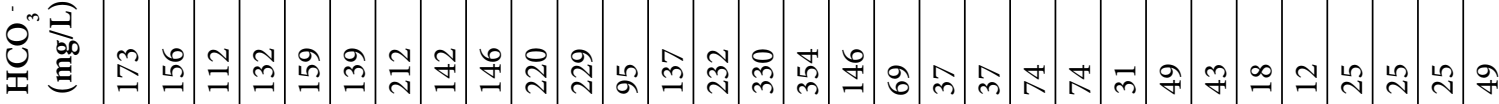

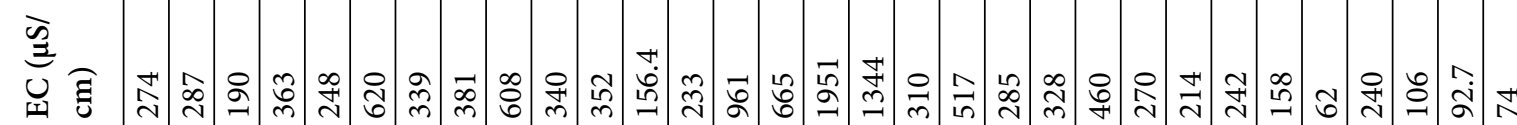

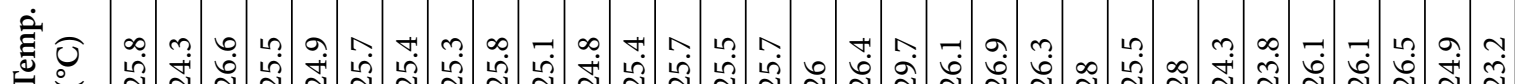

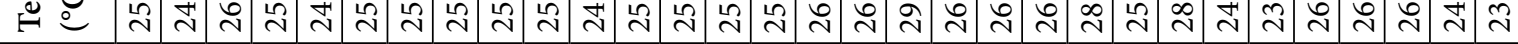

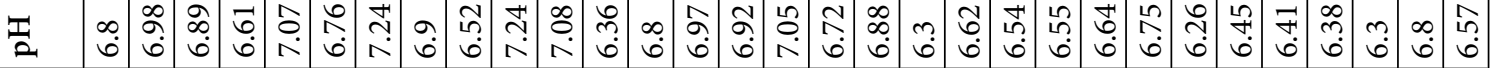

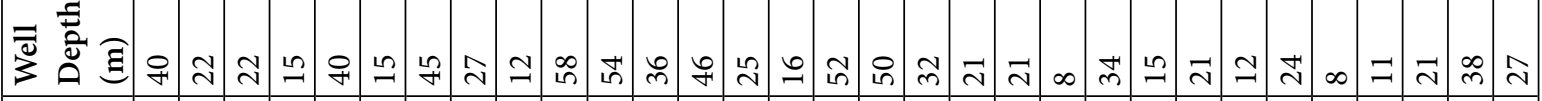

\begin{tabular}{|c|c|c|c|c|c|c|c|c|c|c|c|c|c|c|c|c|c|c|c|c|c|c|c|c|c|c|c|c|c|c|c|c|}
\hline$\stackrel{\mathscr{J}}{4}$ & & ( & $\frac{3}{2}$ & 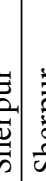 & 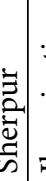 & | & $\frac{\pi}{\frac{\pi}{\pi}}$ & 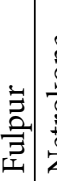 & 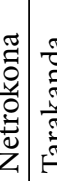 & 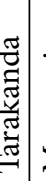 & 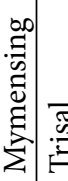 & 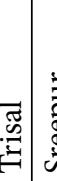 & 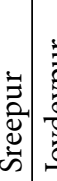 & 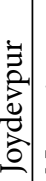 & : & 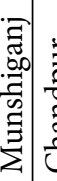 & 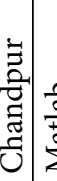 & 莒 & 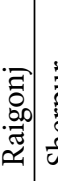 & 壳 & 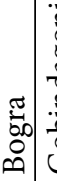 & 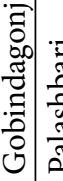 & 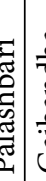 & 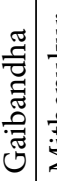 & 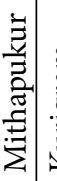 & - & 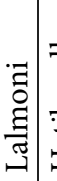 & 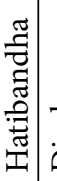 & 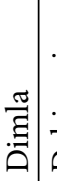 & 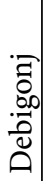 & 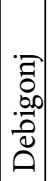 & 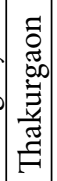 \\
\hline
\end{tabular}

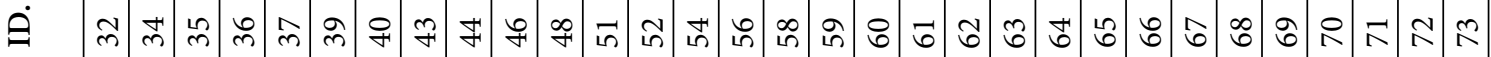


군

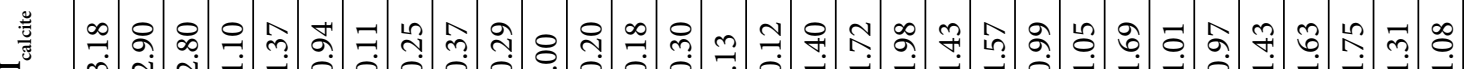

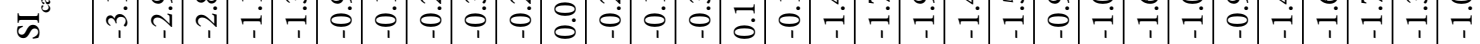

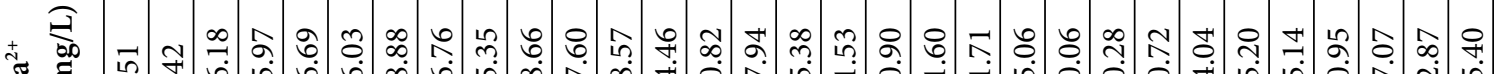

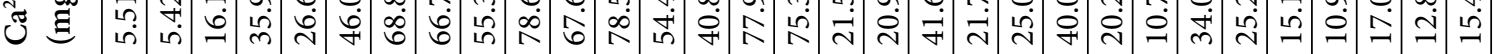

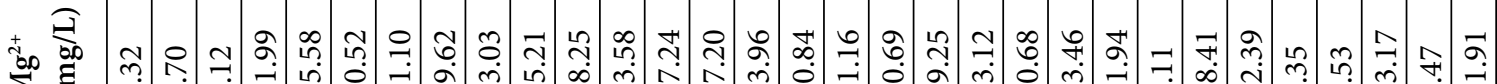

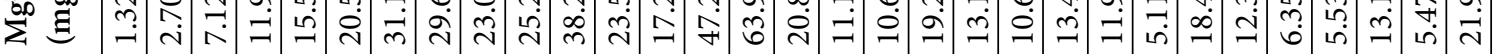

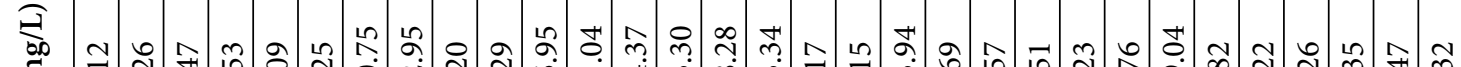

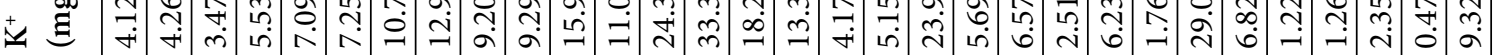

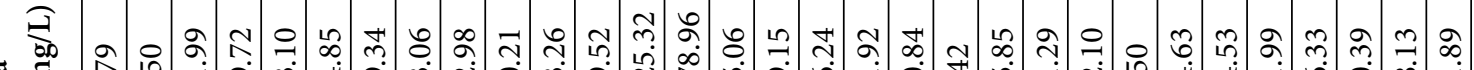
艺

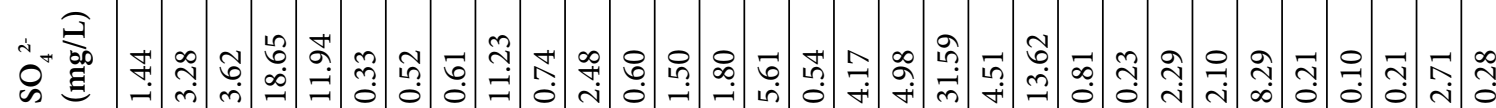

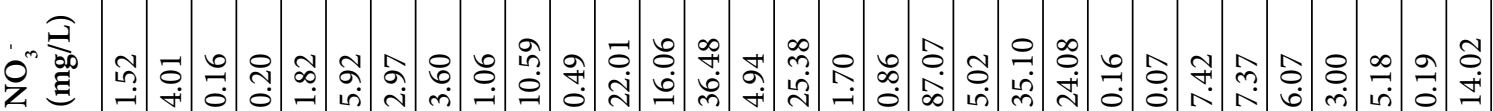

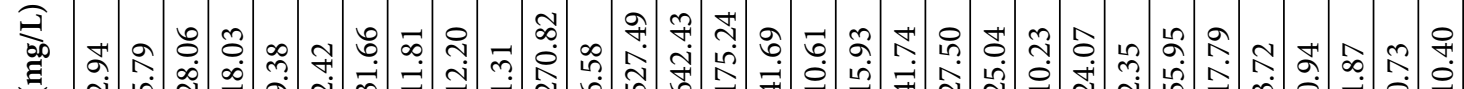

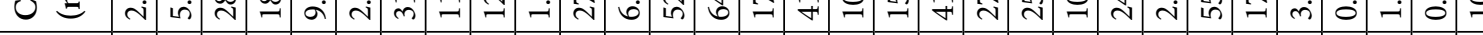

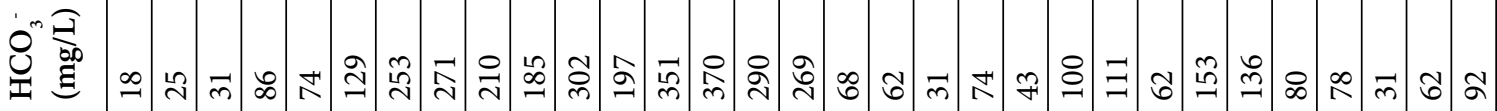

坣

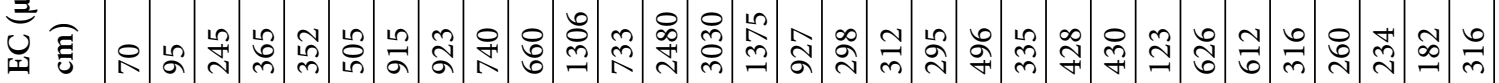

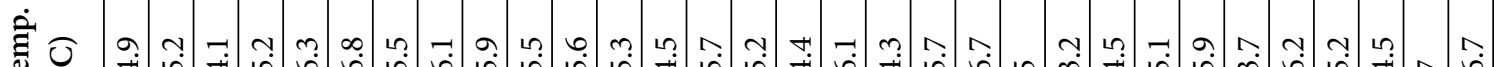

ॠ

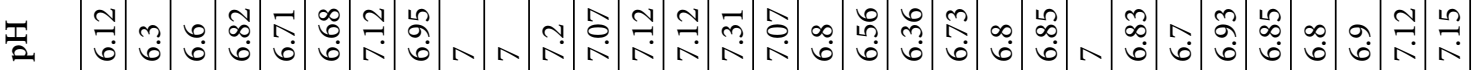

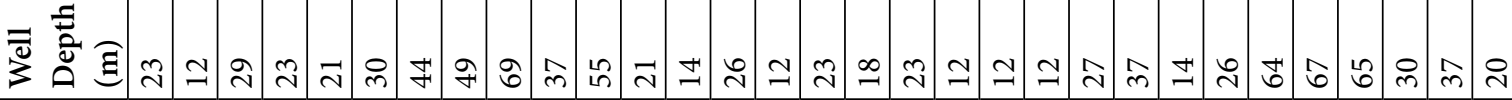

\begin{tabular}{|c|c|c|c|c|c|c|c|c|c|c|c|c|c|c|c|c|c|c|c|c|c|c|c|c|c|c|c|}
\hline 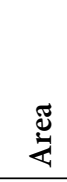 & $\begin{array}{l}0 \\
0 \\
0 \\
0 \\
0\end{array}$ & & $\begin{array}{l}\mid \tilde{0} \\
\tilde{0} \\
0 \\
\tilde{\sigma} \\
z \\
\end{array}$ & 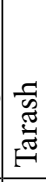 & 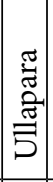 & 㞼 & 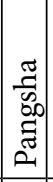 & & 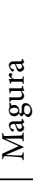 & 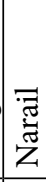 & 5 & 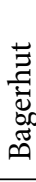 & 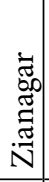 & 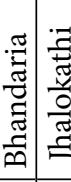 & 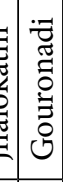 & 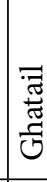 & 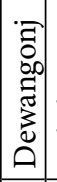 & 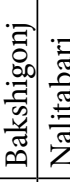 & 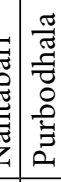 & : & 孠 & 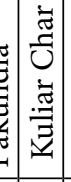 & 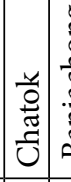 & 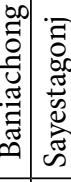 & 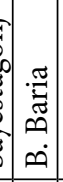 & 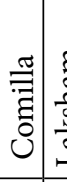 & 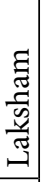 \\
\hline$\theta$ & I & & 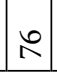 & $\hat{\wedge}$ & & 2 & $\infty$ & $\infty$ & $\infty$ & $\infty$ & $\infty$ & & $\infty$ & ১ ৪ & $2 \pi$ & & J゙ & 2ૂ รั & & $\infty$ & 2 & $\overrightarrow{0}$ & 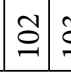 & 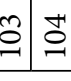 & $\stackrel{2}{2}$ & 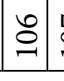 & \\
\hline
\end{tabular}




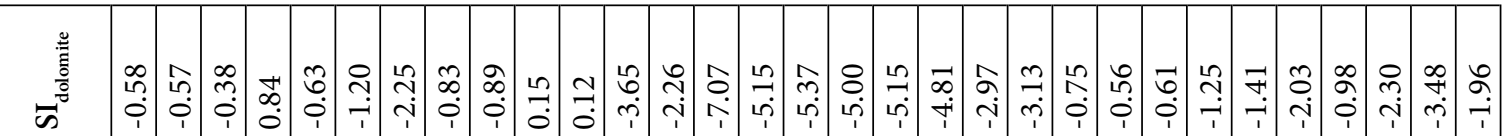

竞

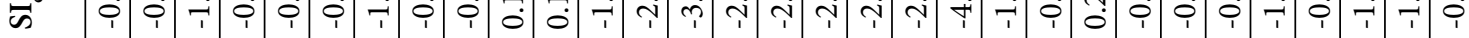

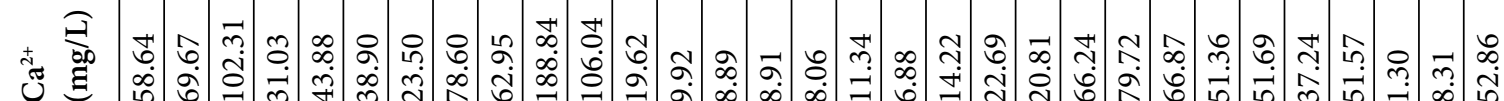

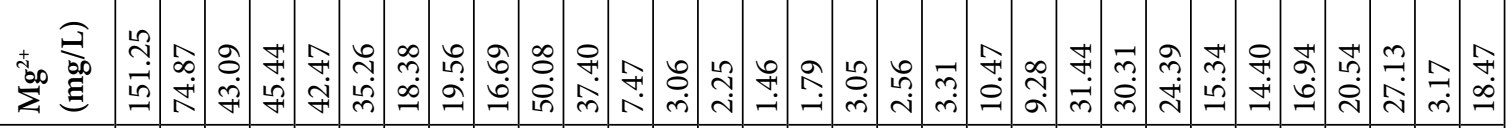

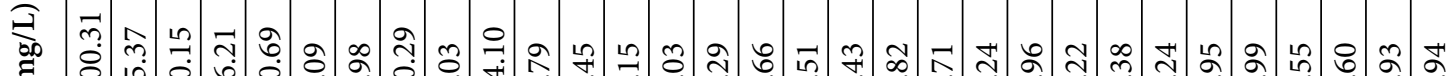

去 费

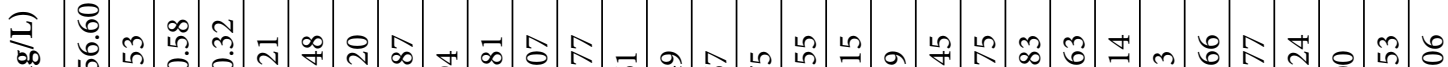

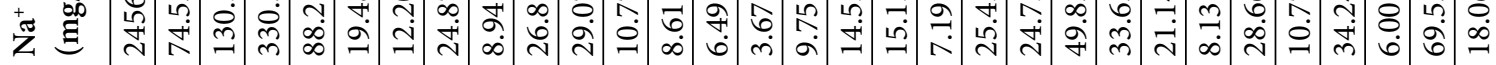

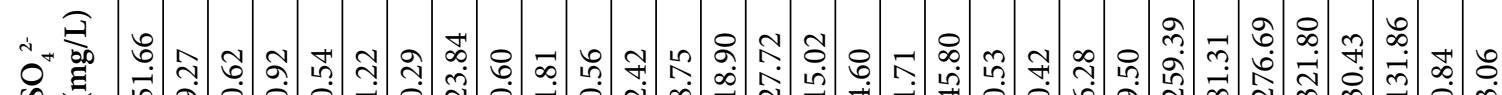

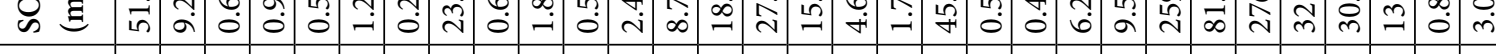

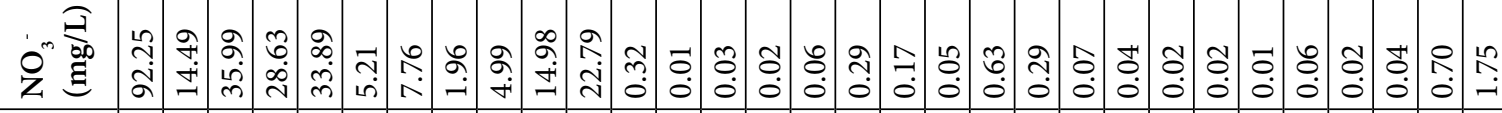

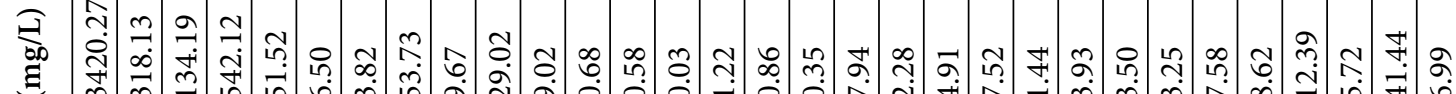

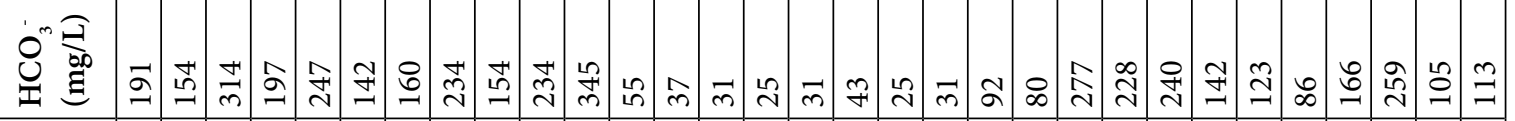

岱

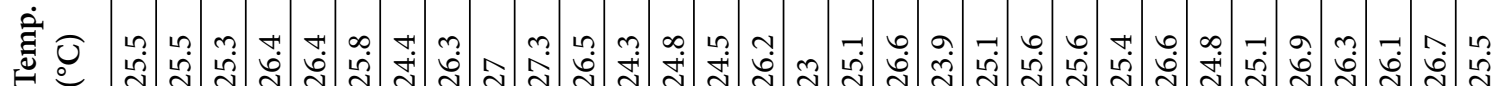

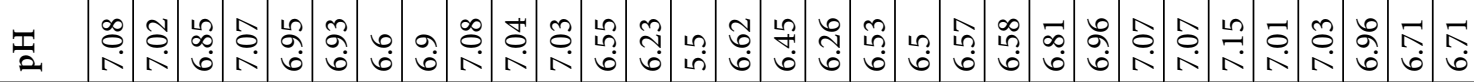

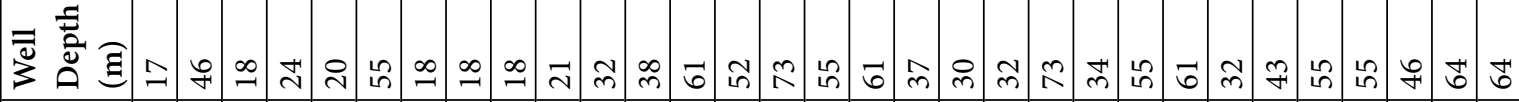

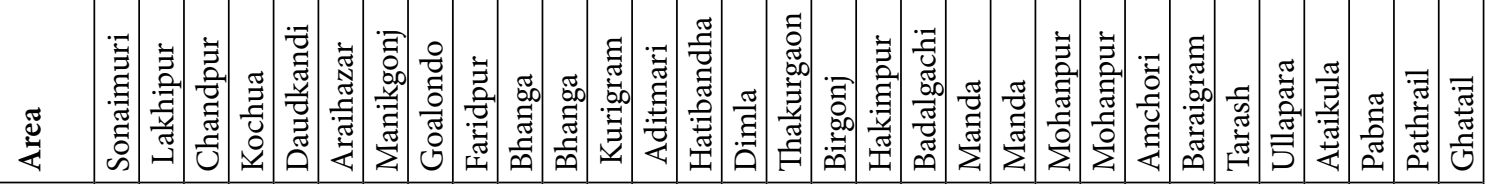

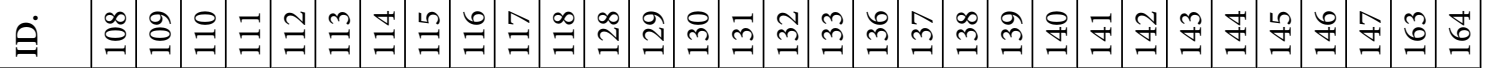




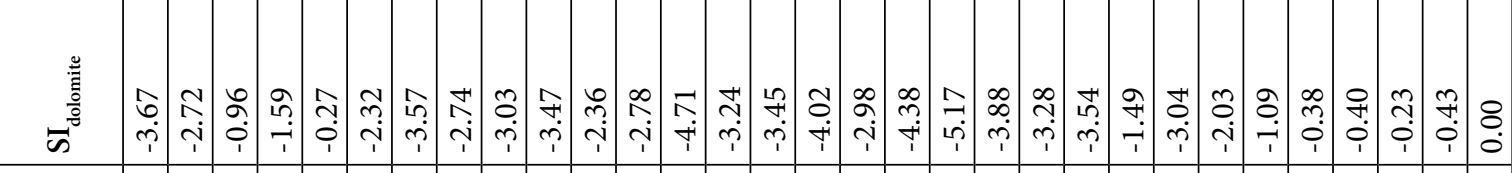

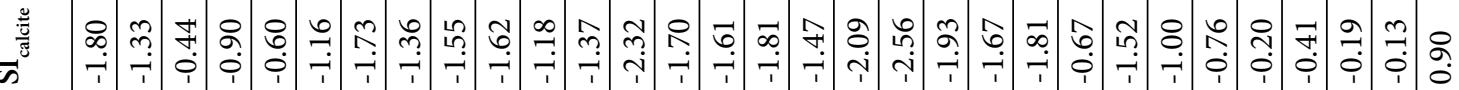

东

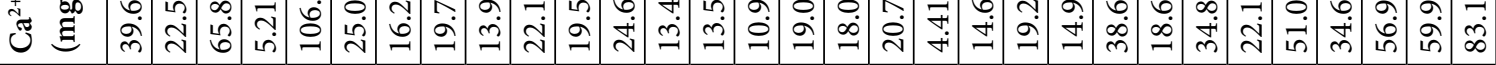

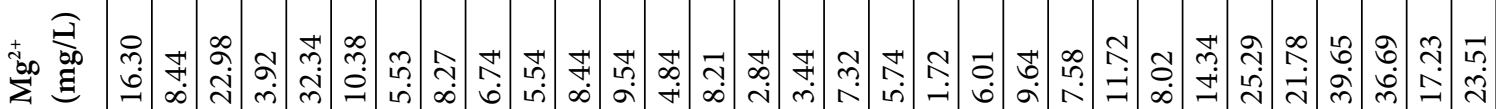

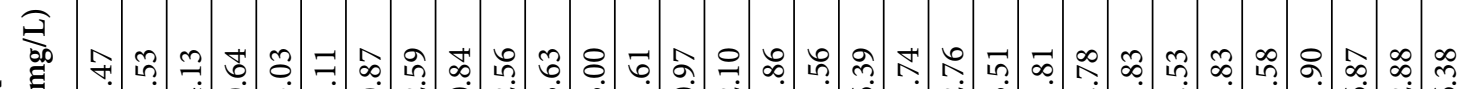

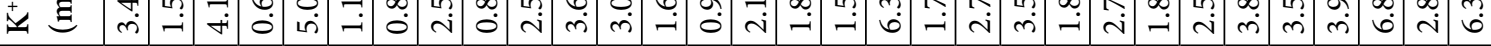

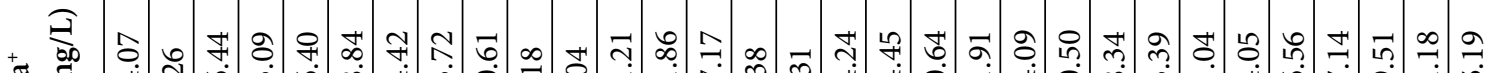

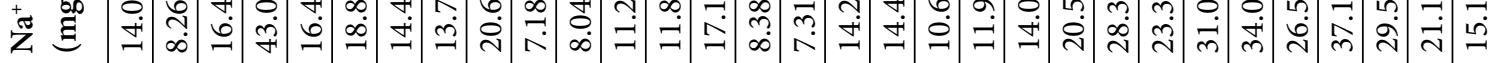

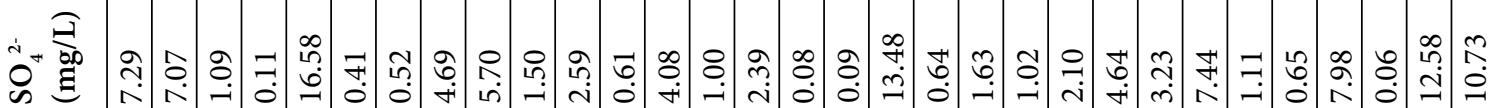

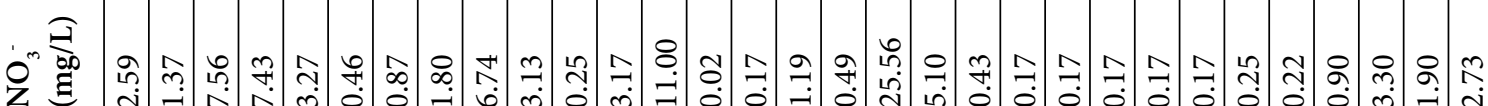

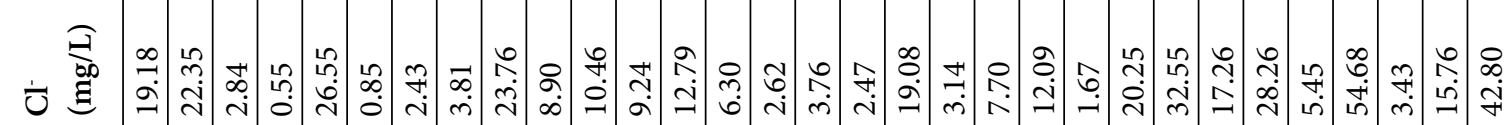

考察

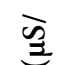

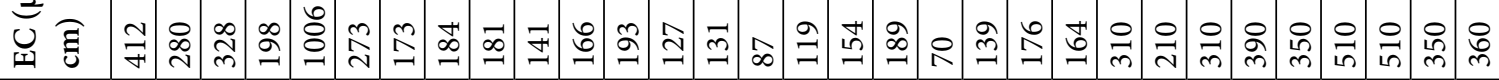

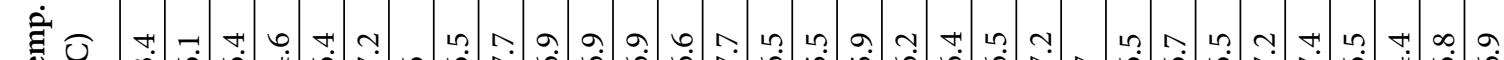
焉

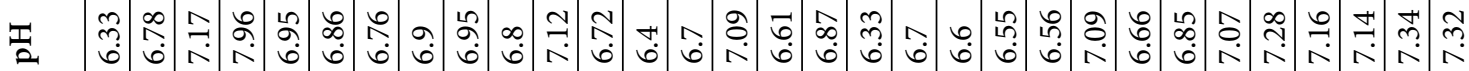

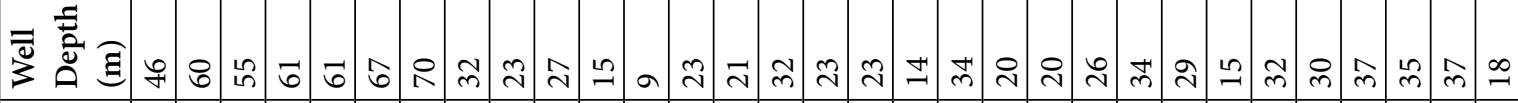

\begin{tabular}{|c|c|c|c|c|c|c|c|c|c|c|c|c|c|c|c|c|c|c|c|c|c|c|c|c|c|c|}
\hline$\stackrel{\Xi}{\square}$ & 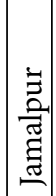 & 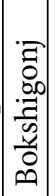 & 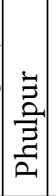 & 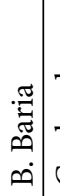 & 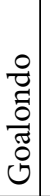 & $\begin{array}{l}\frac{\pi}{3} \\
\frac{\pi}{\tilde{M}}\end{array}$ & $\begin{array}{l}\frac{\pi}{3} \\
\frac{3}{\tilde{J}} \\
\frac{\pi}{\infty}\end{array}$ & 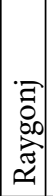 & 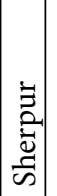 & & 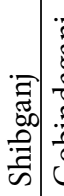 & 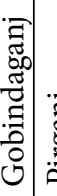 & 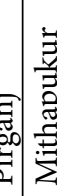 & 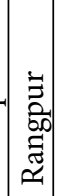 & 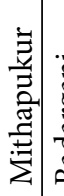 & 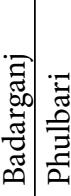 & 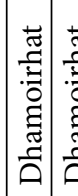 & 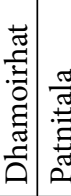 & 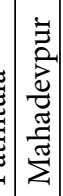 & 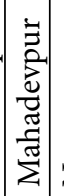 & 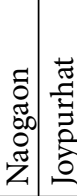 & 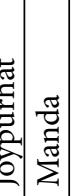 & : & 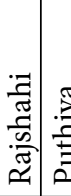 & 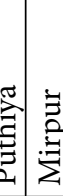 & $\left\{\begin{array}{l}\vec{Z} \\
\text { ర్ } \\
\widetilde{\Xi}\end{array}\right.$ \\
\hline & & $\underset{0}{0}$ & $\begin{array}{l}\infty \\
0 \\
0\end{array}$ & 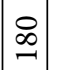 & $\Omega$ & & o & & $\vec{\sim}$ & & ְ̊స & 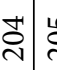 & 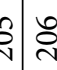 & ڤి & 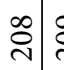 & ڤેे & \begin{tabular}{l|l}
$\stackrel{\sim}{\sim}$ & \multirow{2}{*}{}
\end{tabular} & 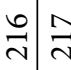 & $\vec{\sim}=\frac{\infty}{\sim}$ & $\stackrel{\vartheta}{\vec{\nu}}$ & సి & 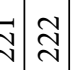 & $\stackrel{\approx}{\approx}$ & $\underset{\mathbb{N}}{\stackrel{\Delta}{2}}$ & & $\stackrel{\infty}{\sim}$ \\
\hline
\end{tabular}




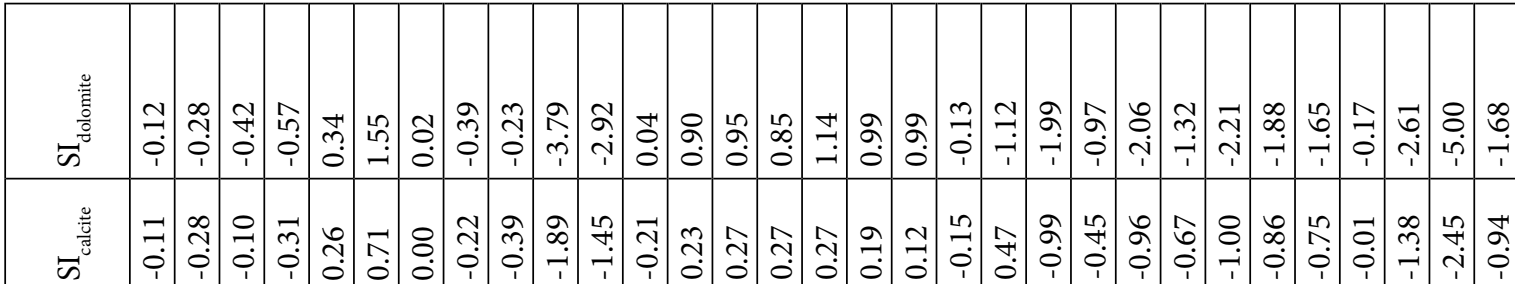

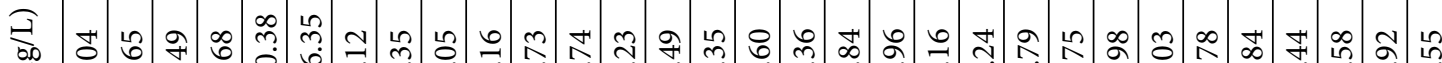
ঊ゙

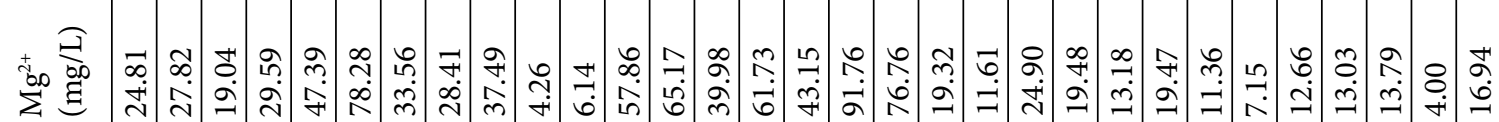

皇

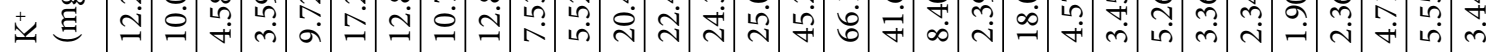

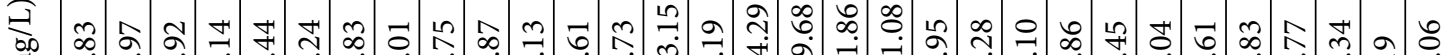

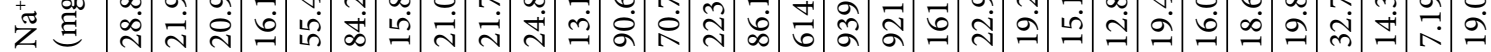

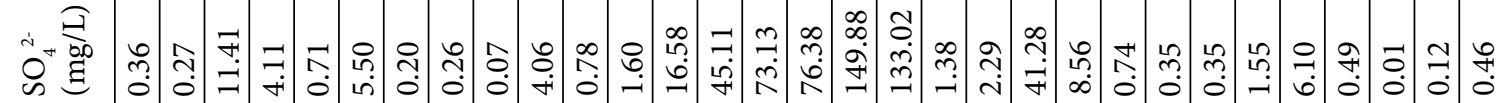

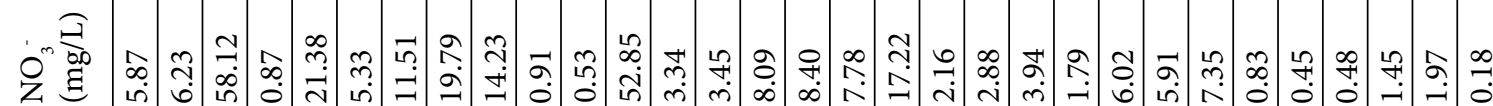

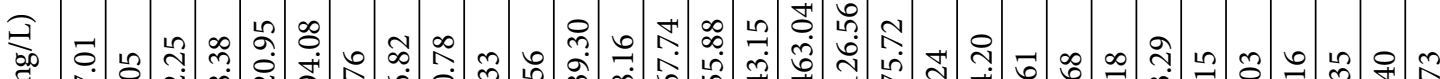

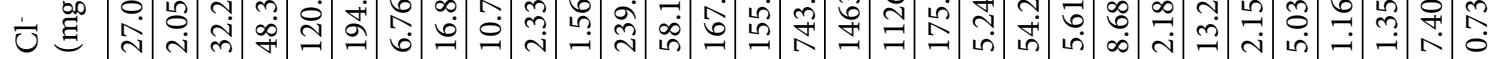

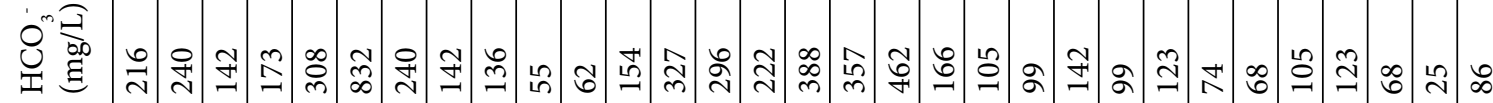
$\underset{3}{3}$

ن্工

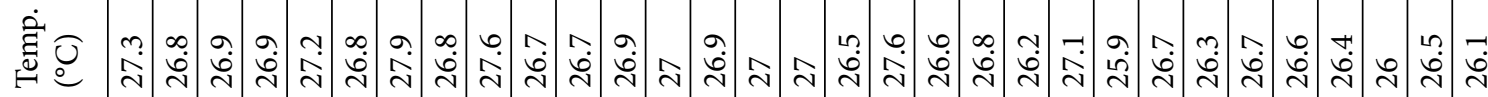

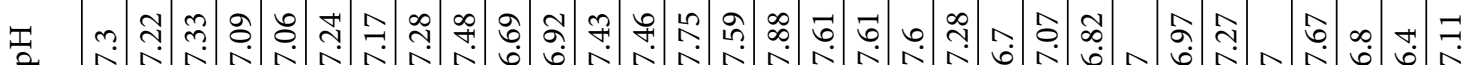

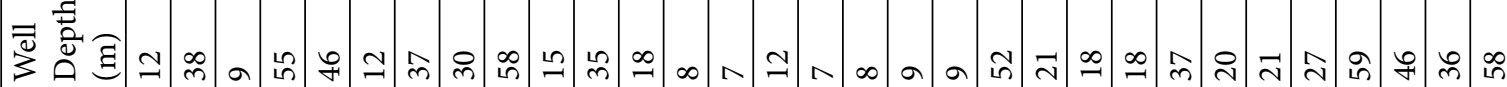

\begin{tabular}{|c|c|c|c|c|c|c|c|c|c|c|c|c|c|c|c|c|c|c|c|c|c|c|c|c|c|c|c|c|c|c|}
\hline$\stackrel{\mathbb{Z}}{\stackrel{\mathbb{Z}}{4}}$ & 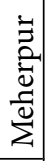 & 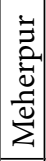 & 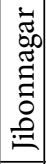 & 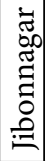 & 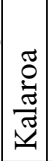 & 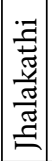 & 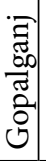 & 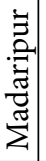 & 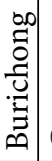 & 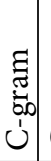 & 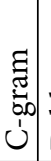 & 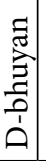 & 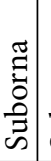 & 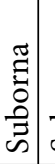 & 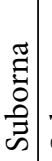 & 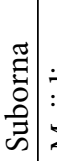 & $\stackrel{\bar{\Xi}}{:=}$ & 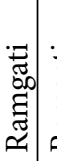 & 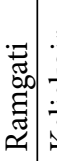 & 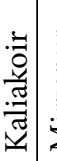 & 营 & 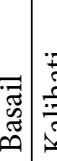 & 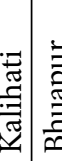 & 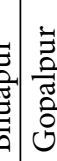 & 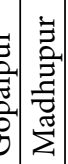 & 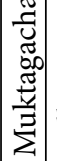 & 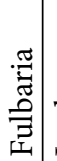 & 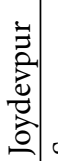 & : & 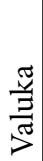 \\
\hline$\dot{\theta}$ & 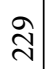 & $\stackrel{\overbrace{}}{\sim}$ & $\tilde{\sim}$ & $\tilde{\sim}$ & 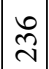 & $\underset{\sim}{\stackrel{Z}{Z}}$ & 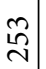 & $\begin{array}{l}\infty \\
\stackrel{\sim}{\sim}\end{array}$ & ָे & 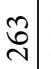 & $\begin{array}{l}\stackrel{D}{0} \\
\text { | }\end{array}$ & 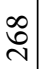 & 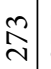 & ડิ & શิ & $\underset{\sim}{\sim}$ & 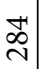 & $\begin{array}{l}\infty \\
\infty \\
\sim \\
\sim\end{array}$ & $\overline{\mathrm{\sigma}}$ & ปે & ๙ે & ন্ & ๙ั) & م̂ & $\overrightarrow{\mathrm{n}}$ & రి & D্ & $\begin{array}{l}\infty \\
0 \\
\text { ల) }\end{array}$ & gे & $\frac{0}{m}$ \\
\hline
\end{tabular}




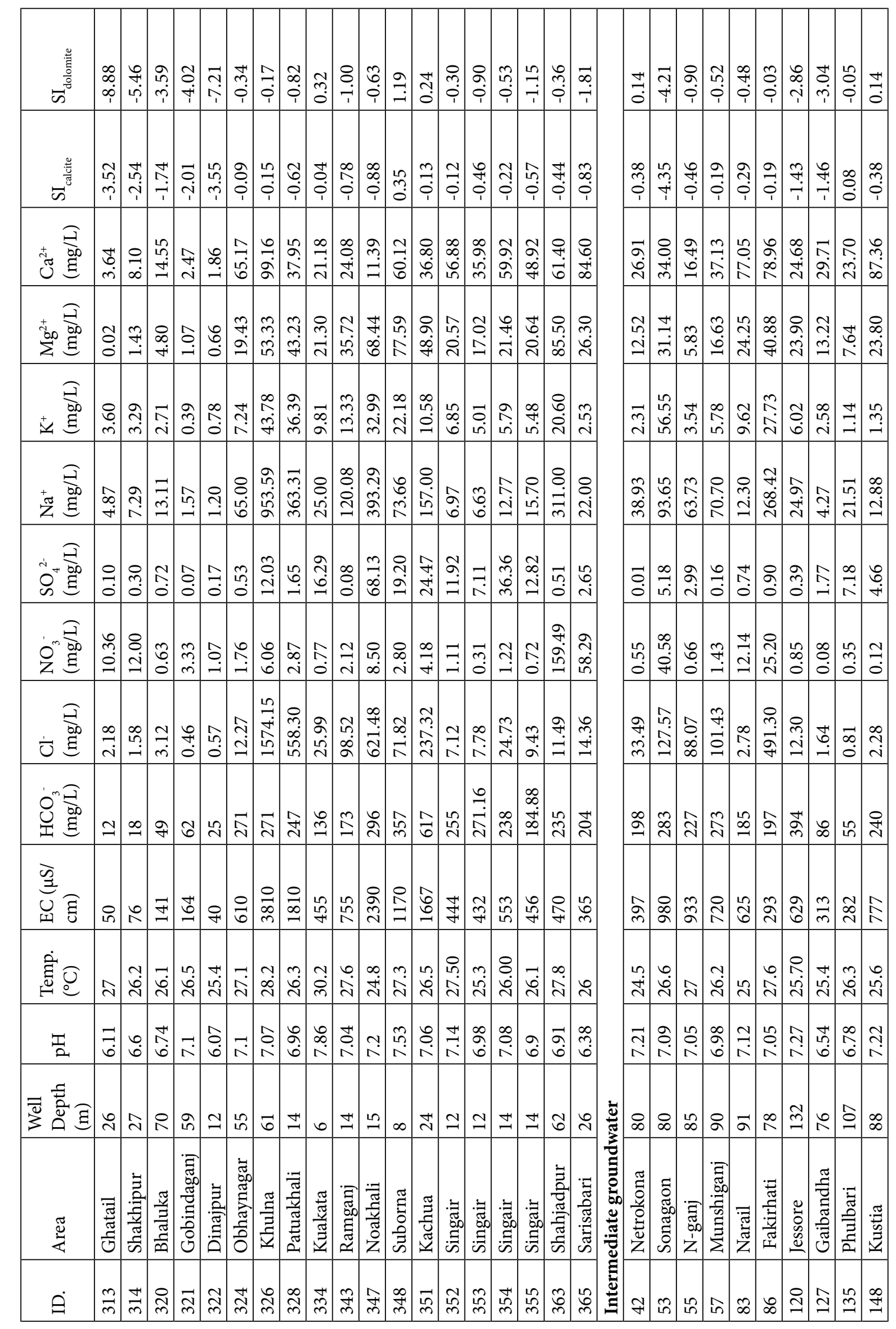




\begin{tabular}{|c|c|c|c|c|c|c|c|c|c|c|c|c|c|c|c|c|c|c|c|c|c|c|c|c|c|c|c|c|c|}
\hline $5^{\frac{\bar{c}}{8}}$ & : & సి & & $\overbrace{0}^{8}$ & is & 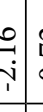 & $\begin{array}{l}\tilde{N} \\
0 \\
1\end{array}$ & ț & 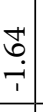 & $\begin{array}{l}\stackrel{i}{1} \\
i \\
\end{array}$ & \begin{tabular}{c}
$\tilde{n}$ \\
\hdashline \\
\end{tabular} & $\mid \begin{array}{c}\vec{\infty} \\
\rightarrow \\
\end{array}$ & $\begin{array}{l}0 \\
\\
i \\
\end{array}$ & 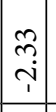 & $\begin{array}{c}0 \\
0 \\
i \\
i\end{array}$ & 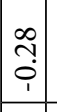 & $\begin{array}{l}0 \\
\vdots \\
0 \\
\end{array}$ & $\begin{array}{c}1 \\
0 \\
0 \\
1\end{array}$ & $\begin{array}{l}8 \\
i \\
i \\
\end{array}$ & & $\begin{array}{l}+\infty \\
\dot{0}\end{array}$ & $\begin{array}{l}n \\
0 \\
0 \\
i\end{array}$ & $\mid \begin{array}{c}\hat{y} \\
\hat{i} \\
i\end{array}$ & 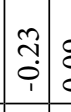 & oे. & 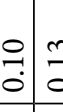 & & है & $\frac{7}{0}$ \\
\hline$\omega^{\frac{2}{\frac{2}{5}}}$ & $\stackrel{0}{\infty}$ & i & $\begin{array}{l}0 \\
0 \\
0 \\
1\end{array}$ & $\frac{a}{7}$ & 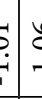 & . & $\begin{array}{c}0 \\
\text { ê. } \\
\end{array}$ & & $\begin{array}{l}1 \\
0 \\
0 \\
0 \\
\end{array}$ & $\begin{array}{l}0 \\
\\
\end{array}$ & 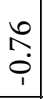 & $\begin{array}{l}0 \\
0 \\
\dot{i} \\
\end{array}$ & 角 & $\begin{array}{c}m \\
\stackrel{2}{-1} \\
\end{array}$ & \begin{tabular}{|l}
2 \\
2 \\
0 \\
1
\end{tabular} & $\begin{array}{l}n \\
0 \\
0 \\
i \\
\end{array}$ & $\begin{array}{l}\stackrel{n}{n} \\
0 \\
0\end{array}$ & $\begin{array}{l}0 \\
\hat{i} \\
i \\
\end{array}$ & $\begin{array}{l}2 \\
\hat{i} \\
i\end{array}$ & $\begin{array}{l}\infty \\
\substack{j \\
c \\
c}\end{array}$ & 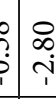 & $\begin{array}{l}m \\
? \\
i \\
1\end{array}$ & 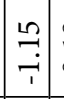 & 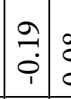 & $\begin{array}{l}\infty \\
0 \\
0 \\
0\end{array}$ & & & & 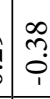 \\
\hline 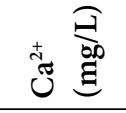 & $\stackrel{\leftrightarrow}{m}$ & 每 & $\begin{array}{l}5 \\
1 \\
7\end{array}$ & ๙ิ & 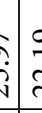 & & مे & $\begin{array}{l}0 \\
\cdots \\
m \\
\end{array}$ & & $\begin{array}{l}\tilde{n} \\
\stackrel{\sim}{2} \\
\end{array}$ & 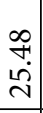 & $\begin{array}{l}\infty \\
\infty \\
1 \\
\\
\end{array}$ & $\begin{array}{l}0 \\
0 \\
\dot{0} \\
\end{array}$ & $\begin{array}{l}1 \\
1 \\
\\
n \\
n\end{array}$ & \begin{tabular}{|l}
$\stackrel{\tilde{m}}{\tilde{j}}$ \\
$\stackrel{\sim}{\sim}$
\end{tabular} & \begin{tabular}{|l|} 
\\
\multirow{2}{*}{} \\
$\stackrel{2}{N}$ \\
\end{tabular} & $\begin{array}{l}\hat{2} \\
\grave{2} \\
\end{array}$ & $\begin{array}{l}\mathrm{N} \\
\stackrel{N}{\mathrm{~N}} \\
\end{array}$ & 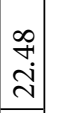 & $\begin{array}{ll}7 \\
7 \\
10 \\
\end{array}$ & 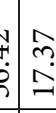 & $\begin{array}{l}\Omega \\
\\
\dot{m}\end{array}$ & $\mid \begin{array}{c}1 \\
n \\
0 \\
m\end{array}$ & 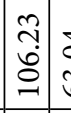 & $\begin{array}{l}\vdots \\
\dot{0} \\
\dot{0}\end{array}$ & & & & 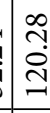 \\
\hline$\sum^{500}$ & 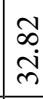 & 离 & $\begin{array}{l}\tilde{N} \\
\tilde{n}\end{array}$ & $=$ & $\begin{array}{l}\text { va } \\
\\
\end{array}$ & & $\vec{a}$ & के & $\begin{array}{l}\text { So } \\
\text { in }\end{array}$ & $\begin{array}{c}m \\
\stackrel{\infty}{\infty}\end{array}$ & $\begin{array}{l}\hat{6} \\
\dot{\Xi} \\
=\end{array}$ & กิ? & 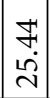 & $\begin{array}{c}a \\
\sigma \\
\sigma\end{array}$ & $\left|\begin{array}{c}\infty \\
\infty \\
\infty\end{array}\right|$ & $\mid$ & 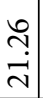 & $\left|\begin{array}{l}8 \\
9 \\
6\end{array}\right|$ & $\left|\begin{array}{c}\infty \\
1 \\
\infty\end{array}\right|$ & 守 & 严 & 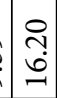 & $\left|\begin{array}{l}\tilde{N} \\
\tilde{n}\end{array}\right|$ & $\mid \begin{array}{c}2 \\
\dot{a} \\
\dot{q}\end{array}$ & $\begin{array}{l}\stackrel{2}{0} \\
\dot{m}\end{array}$ & & & & : \\
\hline \pm & $\stackrel{\sqrt[n]{n}}{i}$ & m. & D & $\begin{array}{l}8 \\
6 \\
1\end{array}$ & & & . & . & ָे & . & \begin{tabular}{l}
$?$ \\
\multirow{i}{*}{} \\
\end{tabular} & \begin{tabular}{|l}
1 \\
6 \\
-1 \\
-1 \\
\end{tabular} & $\underset{\vec{T}}{\stackrel{+}{+}}$ & ले & \begin{tabular}{|l}
$\vec{n}$ \\
$\tilde{n}$ \\
\end{tabular} & $\begin{array}{l}5 \\
60 \\
10\end{array}$ & $\begin{array}{l}\hat{乏} \\
+ \\
\end{array}$ & \begin{tabular}{|l|} 
\\
$\dot{n}$ \\
$\dot{r}$
\end{tabular} & $\begin{array}{l}0 \\
0 \\
0 \\
\end{array}$ & $\begin{array}{l}5 \\
\\
\end{array}$ & 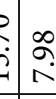 & 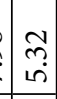 & $\mid \begin{array}{c}i n \\
n \\
\text { n. }\end{array}$ & 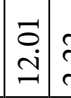 & 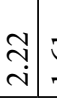 & \begin{tabular}{l|l}
$\overrightarrow{0}$ & 7 \\
& 7
\end{tabular} & & & $\begin{array}{l}\square \\
\infty \\
\infty\end{array}$ \\
\hline 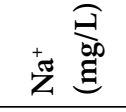 & ๙2. & f & $\frac{\pi}{\pi}$ & ¿ & 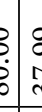 & ริ & 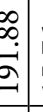 & $\begin{array}{l}0 \\
2 \\
1 \\
1 \\
\end{array}$ & 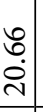 & $\begin{array}{l}\text { bे } \\
\vdots \\
\vdots \\
\end{array}$ & $\begin{array}{l}\text { f. } \\
\text { ம். } \\
\end{array}$ & $\begin{array}{c}n \\
\hat{6} \\
\end{array}$ & $\begin{array}{l}\infty \\
0 \\
\tilde{n} \\
\end{array}$ & $\begin{array}{l}\text { 9 } \\
\text { iे } \\
\text { î. }\end{array}$ & $\begin{array}{l}2 \\
\tilde{n} \\
\end{array}$ & $\begin{array}{l}\infty \\
-\infty \\
-2 \\
\end{array}$ & 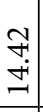 & $\begin{array}{l}0 \\
2 \\
\end{array}$ & \begin{tabular}{c}
0 \\
0 \\
$\infty$ \\
\hdashline \\
\end{tabular} & $\begin{array}{l}15 \\
\text { ले } \\
\end{array}$ & ?2. & $\begin{array}{l}6 \\
\dot{f} \\
\dot{7}\end{array}$ & \begin{tabular}{|l|}
0 \\
$\infty$ \\
$\infty$ \\
$-\infty$ \\
-1
\end{tabular} & 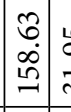 & $\begin{array}{l}\stackrel{n}{2} \\
\dot{m}\end{array}$ & & & กิ & \begin{tabular}{l}
\multirow{2}{*}{} \\
\multirow{2}{*}{}
\end{tabular} \\
\hline 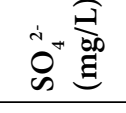 & $\begin{array}{l}\stackrel{+}{\Delta} \\
\infty\end{array}$ & $\begin{array}{l}\infty \\
1 \\
i \\
i\end{array}$ & $\begin{array}{l}\infty \\
m \\
m\end{array}$ & 毽 & is & $\begin{array}{l}* \\
\dot{0}\end{array}$ & . & $\begin{array}{l}1 \\
0 \\
0 \\
0\end{array}$ & लि. & $\begin{array}{l}\tilde{n} \\
0 \\
\end{array}$ & $\begin{array}{l}0 \\
0 \\
0\end{array}$ & $\begin{array}{l}+ \\
0 \\
\end{array}$ & $\begin{array}{l}0 \\
0 \\
2 \\
\vdots\end{array}$ & $\begin{array}{l}\infty \\
\infty \\
-\infty \\
\end{array}$ & \begin{tabular}{|l}
$\infty$ \\
\multirow{2}{*}{} \\
0
\end{tabular} & $\begin{array}{c}0 \\
0 \\
\end{array}$ & $\begin{array}{l}\stackrel{n}{2} \\
\dot{m}\end{array}$ & $\begin{array}{l}0 \\
0 \\
0\end{array}$ & $\begin{array}{l}0 \\
\text { in } \\
\text { in } \\
\end{array}$ & 3 & $\begin{array}{l}\hat{y} \\
\end{array}$ & 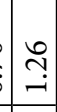 & $\begin{array}{l}0 \\
+ \\
+ \\
\end{array}$ & 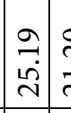 & ָָ. & \begin{tabular}{l|l}
0 & 9 \\
6 & $\infty$ \\
- & $\infty$
\end{tabular} & & & $\stackrel{ \pm}{=}$ \\
\hline $\mathrm{O}^{\infty} \stackrel{5}{500}$ & $\stackrel{2}{0}$ & $\stackrel{2}{\circ}$ & $\begin{array}{l}8 \\
0\end{array}$ & $\begin{array}{l}15 \\
15 \\
1\end{array}$ & ๙ૃ & $\begin{array}{lll}R & 1 \\
n & 1 & \end{array}$ & â. & $\begin{array}{c}m \\
0 \\
0\end{array}$ & 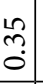 & 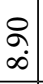 & $\begin{array}{l}\infty \\
0 \\
0 \\
\end{array}$ & $\begin{array}{l}\stackrel{\vartheta}{\hat{i}} \\
i\end{array}$ & $\mid \begin{array}{l}m \\
\dot{m} \\
\dot{m}\end{array}$ & $\begin{array}{l}+ \\
0 \\
0\end{array}$ & กุ? & \begin{tabular}{|l}
\multirow{b}{*}{} \\
in
\end{tabular} & $\begin{array}{l}\text { L } \\
\text { in } \\
\end{array}$ & 定 & $\begin{array}{l}0 \\
\text { L̊n. } \\
0\end{array}$ & $\begin{array}{l}\infty \\
\infty \\
\vec{\sigma}\end{array}$ & 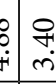 & ఫ: & $\begin{array}{l}\infty \\
\stackrel{1}{-} \\
-\end{array}$ & 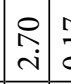 & $\stackrel{-1}{0}$ & 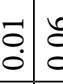 & 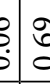 & & $\begin{array}{l}\dot{1} \\
\vdots \\
\vdots\end{array}$ \\
\hline$\dot{0} \stackrel{\widehat{\vec{\theta}}}{\stackrel{\Xi}{\Xi}}$ & $\begin{array}{l}8 \\
\pm \\
\end{array}$ & $\stackrel{\text { ț }}{-}$ & $\begin{array}{l}0 \\
\infty \\
0 \\
0\end{array}$ & 2 & 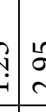 & $\hat{\text { र̂t }}$ & $\begin{array}{c}n \\
\text { L } \\
\end{array}$ & م. & $\begin{array}{l}\vec{b} \\
\dot{m}\end{array}$ & $\begin{array}{l}2 \\
\vdots \\
0 \\
0\end{array}$ & ஸे. & $\begin{array}{l}\tilde{n} \\
\tilde{n} \\
\\
\end{array}$ & \begin{tabular}{|l|}
8 \\
0 \\
0 \\
\end{tabular} & $\begin{array}{l}\infty \\
\stackrel{1}{1} \\
\end{array}$ & 离 & $\begin{array}{c}\tilde{N} \\
\text { in } \\
\end{array}$ & $\begin{array}{l}2 \\
0 \\
0\end{array}$ & $\begin{array}{l}0 \\
\text { i } \\
\end{array}$ & $\begin{array}{l}\stackrel{R}{2} \\
\\
\end{array}$ & $\stackrel{\infty}{\frac{\infty}{n}}$ & 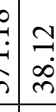 & \begin{tabular}{|l}
$\alpha$ \\
$\dot{+}$
\end{tabular} & $\mid \begin{array}{c}0 \\
\stackrel{2}{2} \\
\vec{v}\end{array}$ & 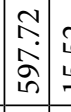 & 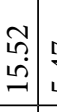 & 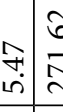 & 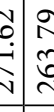 & 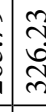 & 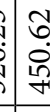 \\
\hline 总 & તิ & 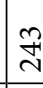 & $\stackrel{2}{n}$ & $\delta$ & 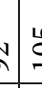 & है) & $\begin{array}{c}\text { in } \\
\text { ते }\end{array}$ & 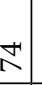 & $\underset{\beth}{ \pm}$ & $\stackrel{\Xi}{\beth}$ & 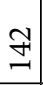 & 点 & $\stackrel{2}{2}$ & $\infty$ & $\infty$ & શิ & $\begin{array}{l}0 \\
\mathrm{v}\end{array}$ & $\stackrel{m}{=}$ & $\infty$ & 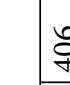 & b & $\stackrel{0}{N}$ & $\underset{\sim}{\stackrel{N}{\sim}}$ & 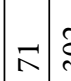 & $\begin{array}{lll}\text { है } & \\
\end{array}$ & ثે & 导 & 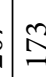 & 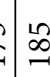 \\
\hline$\bigcup_{i=1}^{0} \widehat{\underline{z}}$ & নু & 왐 & $\frac{10}{9}$ & ભ્ભ & $\hat{~}$ & $\stackrel{n}{n}$ & 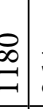 & ఫ్ & $\vec{b}$ & 㐔 & \begin{tabular}{l}
8 \\
\hdashline \\
$i$
\end{tabular} & $\begin{array}{l}+ \\
\forall\end{array}$ & 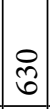 & $\vec{m}$ & હ্ণ & 吕 & 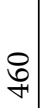 & $\left|\begin{array}{c}8 \\
0 \\
ల\end{array}\right|$ & 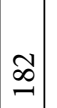 & ț & t) & {$\left[\begin{array}{c}0 \\
i n\end{array}\right.$} & Ñ & $\overrightarrow{\tilde{\sigma}}$ & 突| & 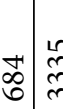 & $\begin{array}{l}\hat{n} \\
\text { nิ }\end{array}$ & in & ì \\
\hline 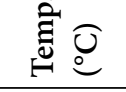 & $\stackrel{\infty}{\stackrel{\infty}{i}}$ & $\begin{array}{l}0 \\
\dot{0} \\
\text { i. }\end{array}$ & ते & 15 & i & H & ते & $\begin{array}{l}\hat{n} \\
\text { cे }\end{array}$ & $\begin{array}{l}\stackrel{+}{\mathrm{i}} \\
\mathrm{N}\end{array}$ & $\stackrel{m}{\grave{n}}$ & $\begin{array}{l}0 \\
\dot{n} \\
\end{array}$ & $\begin{array}{l}\ddot{0} \\
\dot{\omega}\end{array}$ & $\mid \begin{array}{c}n \\
\stackrel{n}{i} \\
\end{array}$ & $\begin{array}{l}0 \\
\stackrel{2}{2}\end{array}$ & 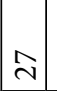 & ָ̊. & 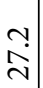 & $\stackrel{\infty}{\sim}$ & $\begin{array}{l}n \\
\stackrel{n}{\sim}\end{array}$ & \begin{tabular}{l}
8 \\
\multirow{2}{n}{}
\end{tabular} & 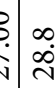 & 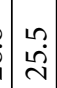 & i & 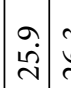 & 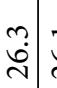 & $\overrightarrow{\text { : }}$ & 3 & $\stackrel{n}{\sim}$ & $?$ \\
\hline 兑 & 웅 & $\exists$ & $\frac{2}{2}$ & $\begin{array}{l}2 \\
\qquad \\
1\end{array}$ & $?$ & $\begin{array}{l}0 \\
0 \\
0\end{array}$ & तै & $\begin{array}{l}0 \\
\vdots \\
\end{array}$ & $\begin{array}{l}0 \\
0 \\
0 \\
0\end{array}$ & $\begin{array}{l}2 \\
\hat{\sigma} \\
\hat{b}\end{array}$ & $\vec{\square}$ & 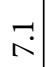 & $\hat{\sigma}$ & 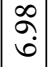 & \begin{tabular}{|l|} 
\\
0 \\
\end{tabular} & 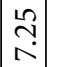 & 矛 & $\stackrel{n}{n}$ & 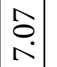 & $\begin{array}{l}0 \\
1\end{array}$ & 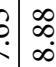 & $\hat{\sigma}$ & 옷 & 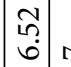 & $\sqrt{1}$ & $\begin{array}{r}+ \\
\end{array}$ & $\begin{array}{l}0 \\
0 \\
\vdots \\
0\end{array}$ & {$\left[\begin{array}{l}\sigma \\
1\end{array}\right.$} & 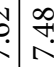 \\
\hline 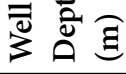 & 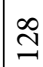 & 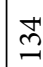 & $\stackrel{\infty}{\sigma}$ & $\stackrel{L}{\infty}$ & $a$ & $\vec{\gamma}$ & $\hat{6}$ & $\infty$ & $\begin{array}{c}\infty \\
0 \\
-1 \\
-1\end{array}$ & $\underset{\Xi}{\mathbb{Z}}$ & ఫै & תี & กี & $\stackrel{\hat{m}}{\sim}$ & 을 & $\Re$ & 응 & 응 & $\sigma$ & 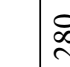 & : & 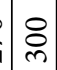 & 永 & 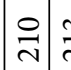 & $\stackrel{m}{\vec{v}}$ & ְ̊: & $\begin{array}{c}0 \\
\vdots \\
5\end{array}$ & $\frac{\circ}{m}$ & 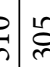 \\
\hline 选 & 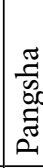 & & : & 吾 & & 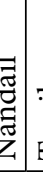 & స్. & 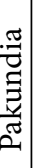 & 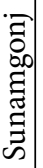 & 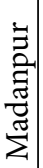 & 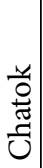 & 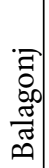 & 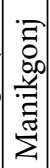 & 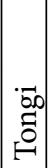 & $\left|\begin{array}{l}\vec{z} \\
0 \\
\vec{Z} \\
\frac{2}{2}\end{array}\right|$ & 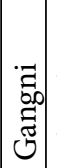 & 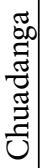 & 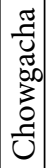 & : & 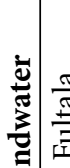 & 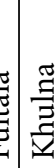 & 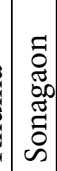 & 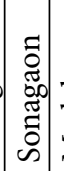 & $\frac{\pi}{3}$ & 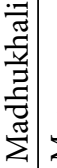 & 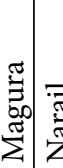 & בֶ: & $\frac{\pi}{2}$ & 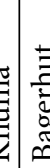 \\
\hline & & n & In & 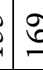 & & & $\approx$ & $\stackrel{\Omega}{\Sigma}$ & 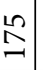 & -1 & 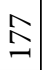 & $\approx$ & $\stackrel{2}{2}$ & Ә & $\mid \vec{\sim}$ & $\widehat{\approx}$ & तి & $\stackrel{\sim}{\approx}$ & 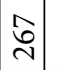 & 离 & $\mathbb{I}$ & $\stackrel{\mathbb{I}}{\stackrel{I}{*}}$ & $\stackrel{\stackrel{\sim}{\Xi}}{\exists}$ & 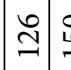 & : & \begin{tabular}{l|l}
$n$ \\
\end{tabular} & $\stackrel{t}{n}$ & 10 & $?$ \\
\hline
\end{tabular}




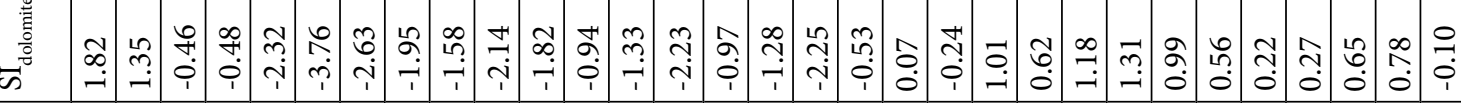

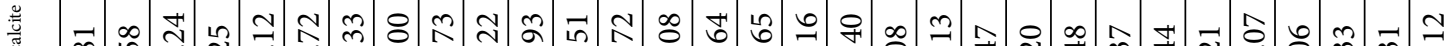

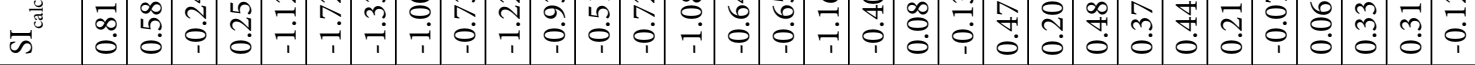

吉 $\underset{60}{0.0}$ f

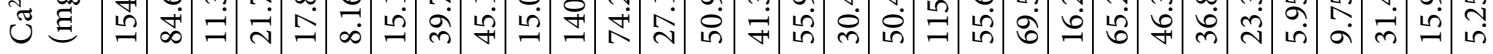

承

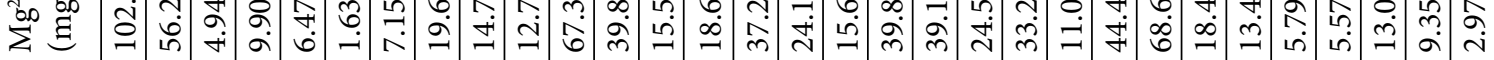

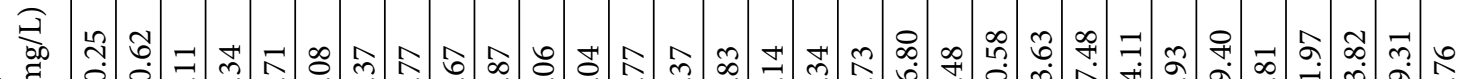

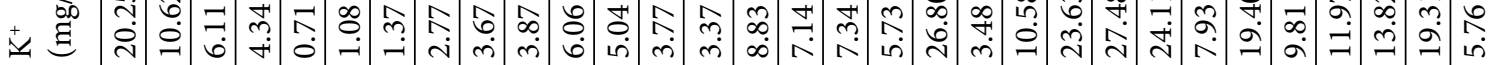

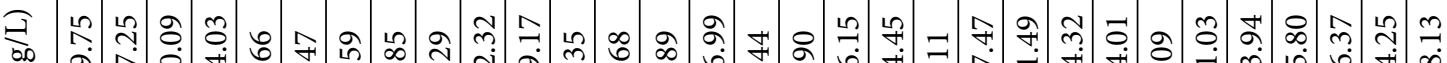

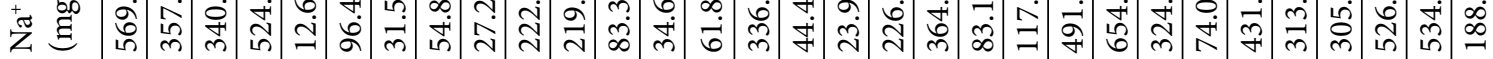

穴定

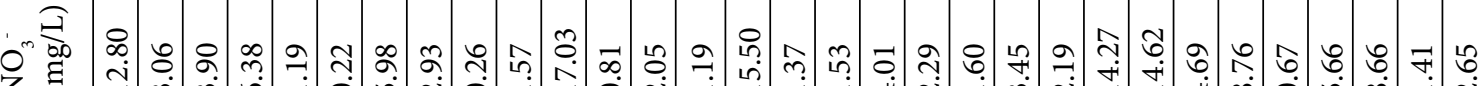

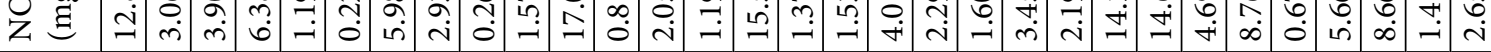

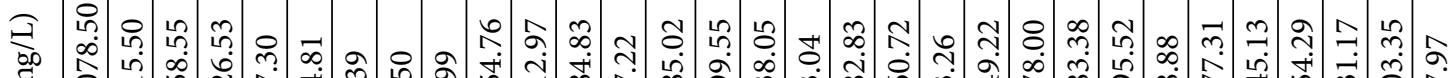

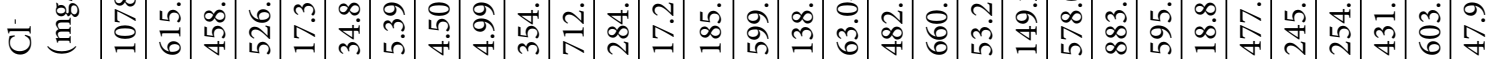

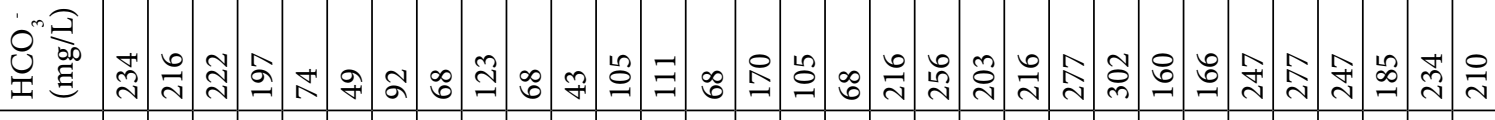

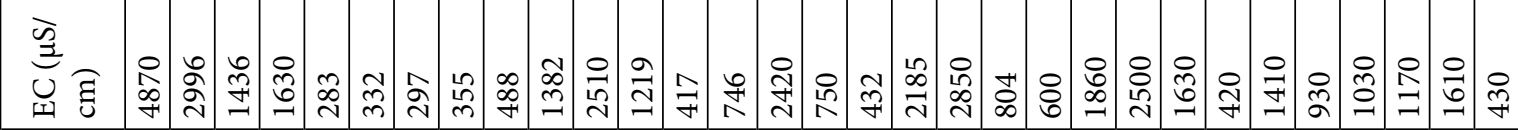

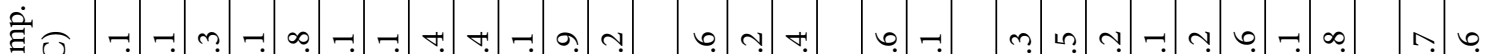

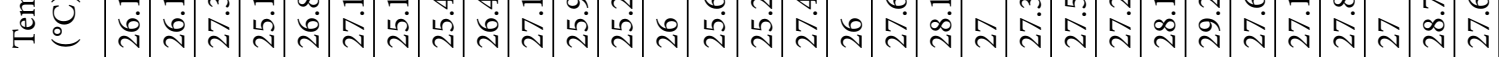

I

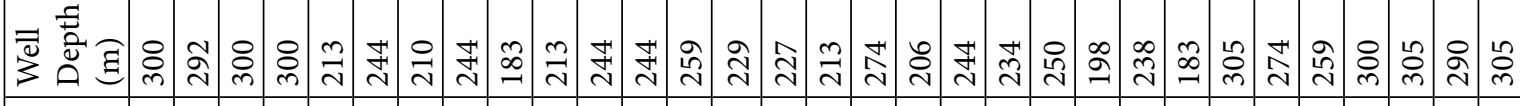

\begin{tabular}{|c|c|c|c|c|c|c|c|c|c|c|c|c|c|c|c|c|c|c|c|c|c|c|c|}
\hline 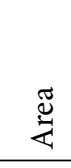 & 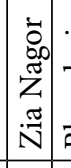 & 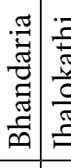 & 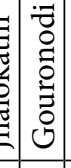 & 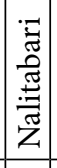 & 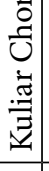 & & 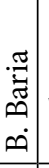 & 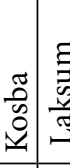 & 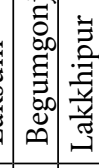 & : & 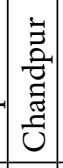 & 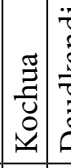 & 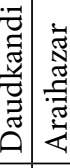 & 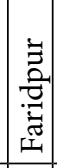 & 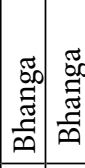 & 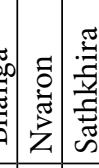 & 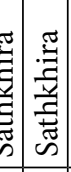 & 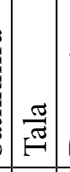 & 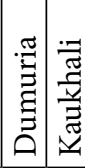 & 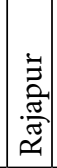 & 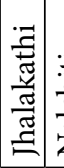 & & 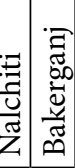 \\
\hline & 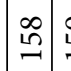 & ถิ & $\vec{\sigma}$ & $\hat{0}$ & & 2 & 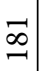 & \begin{tabular}{l|l}
$\infty$ & $\infty$ \\
$\infty$ & $\infty$
\end{tabular} & $\underset{\infty}{\infty}$ & 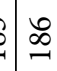 & $\stackrel{\widehat{\infty}}{\infty}$ & \begin{tabular}{|l|l}
$\infty$ & $\&$ \\
$\infty$ &
\end{tabular} & \begin{tabular}{l|l}
$\infty$ & ¿
\end{tabular} & $\stackrel{2}{\Omega}$ & 茴盗 & 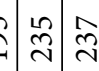 & 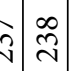 & & 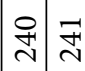 & $\stackrel{\Im}{\sim}$ & $\stackrel{\overbrace{}}{7}$ & $\stackrel{n}{\sim})$ & \begin{tabular}{l}
0 \\
\multirow{1}{*}{} \\
|
\end{tabular} \\
\hline
\end{tabular}




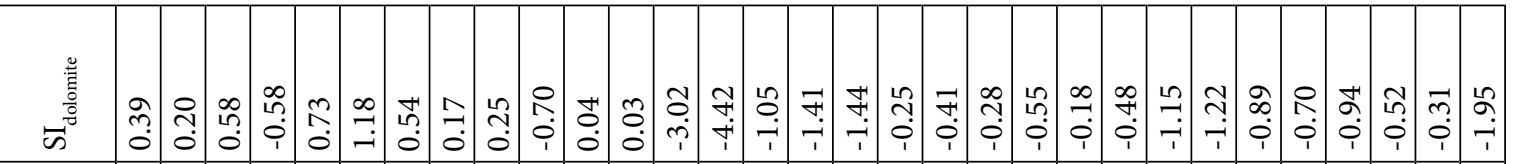

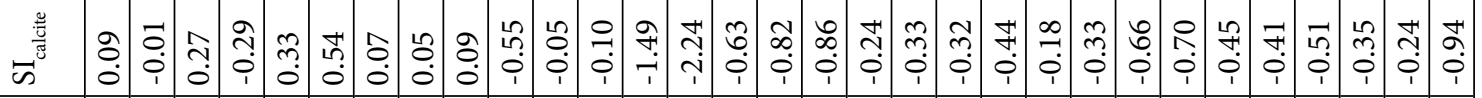

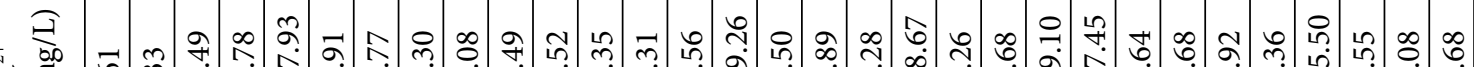

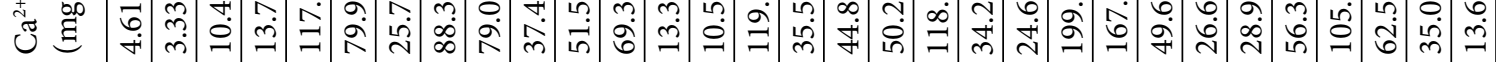

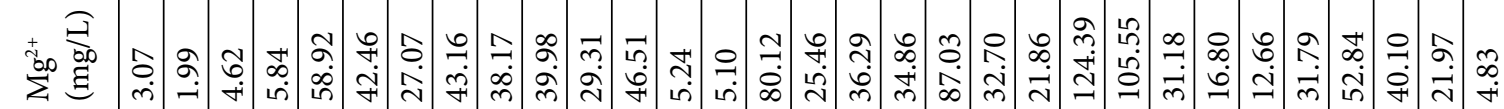

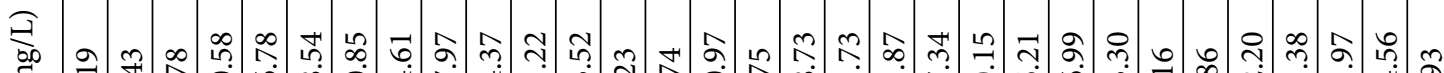

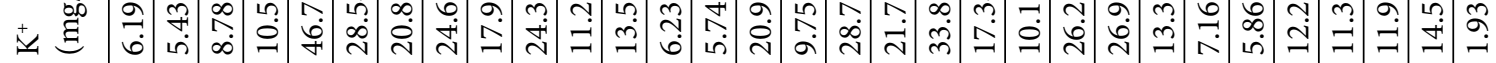

త⿹

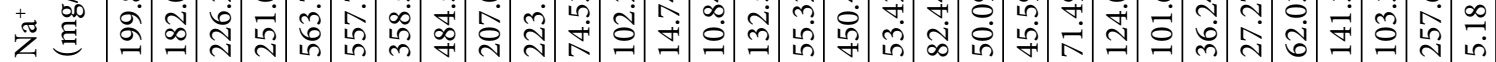

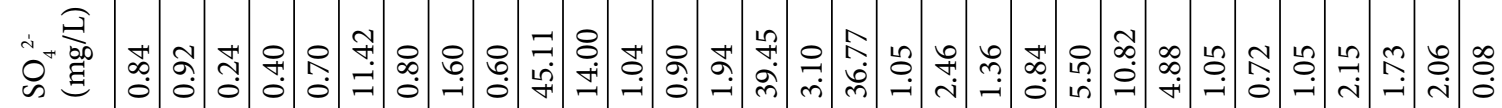

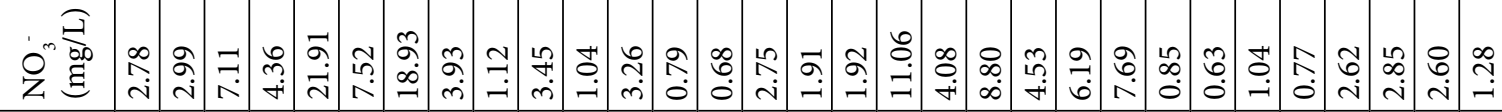

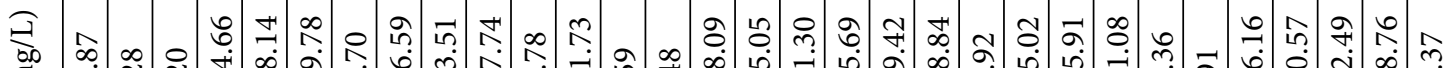

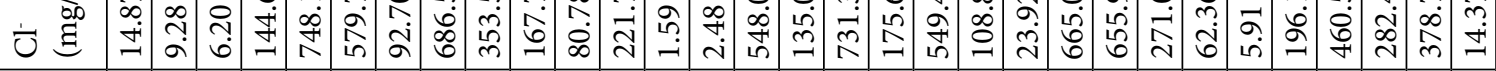

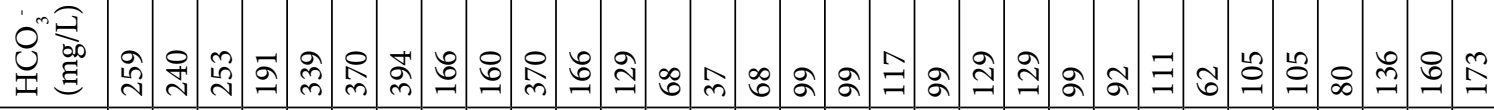
光

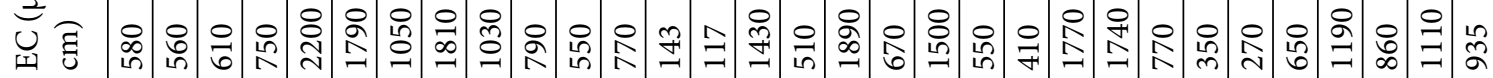

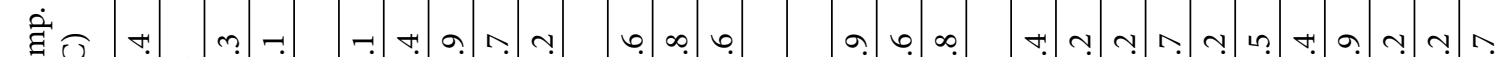

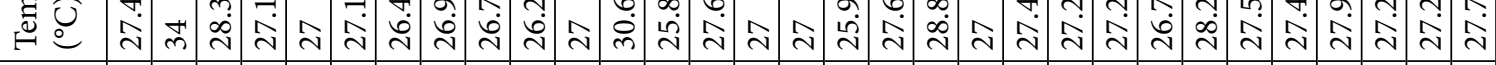

I f f f

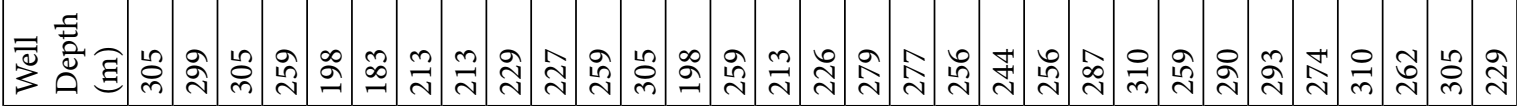

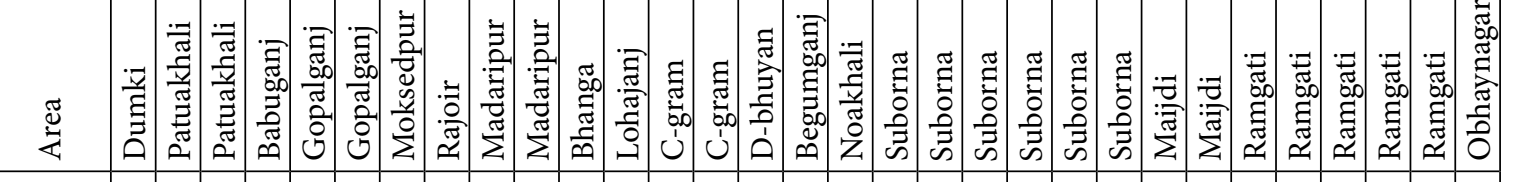

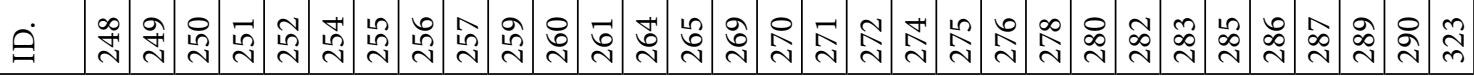




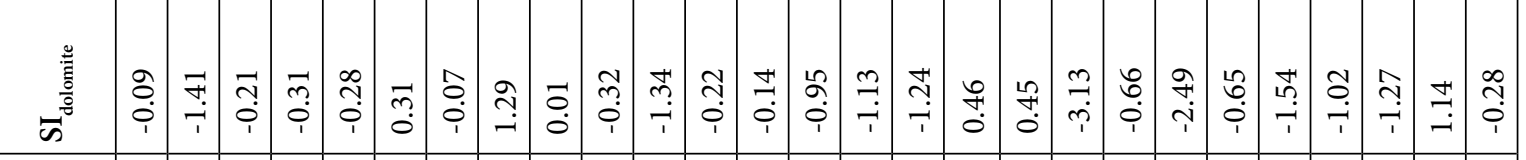

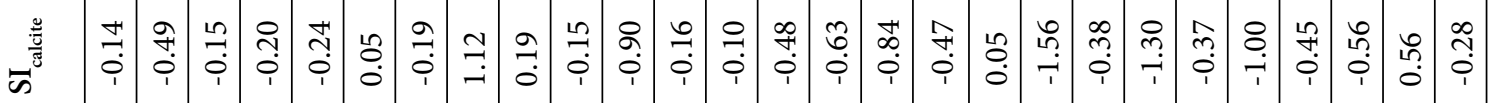

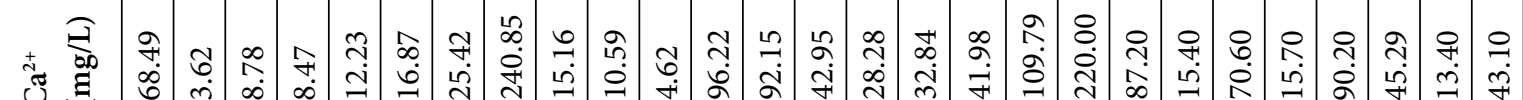

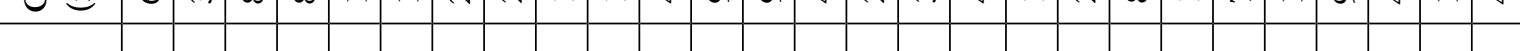

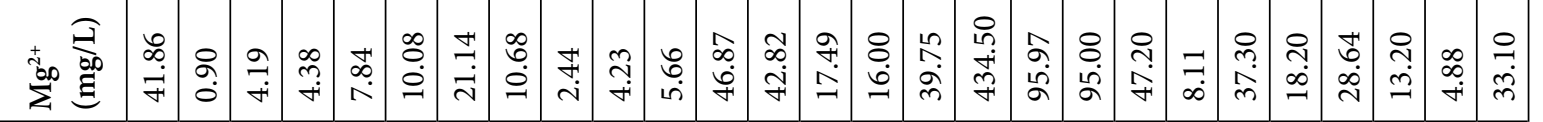

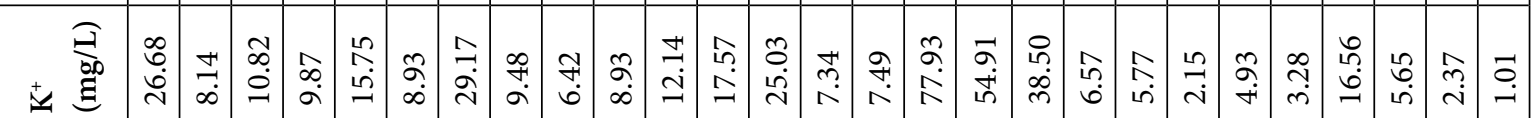

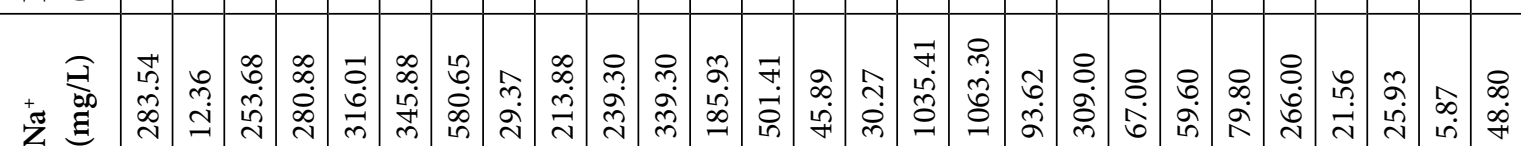

\begin{tabular}{|c|c|c|c|c|c|c|c|c|c|c|c|c|c|c|c|c|c|c|c|c|c|c|c|c|c|}
\hline 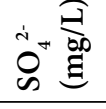 & 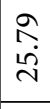 & $\begin{array}{l}\Re \\
\stackrel{0}{0}\end{array}$ & 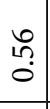 & $\begin{array}{l}\infty \\
0 \\
0 \\
0\end{array}$ & $\overrightarrow{0}$ & $\stackrel{m}{0}$ & $\stackrel{2}{0}$ & $\stackrel{\hat{\sigma}}{-}$ & $\vec{\sigma}$ & ถึ? & 节 & $\hat{\varrho}$ & $\tilde{\overbrace{}}$ & $\stackrel{0}{0}$ & $=$ & & \begin{tabular}{l|l} 
\\
\\
\end{tabular} & 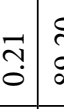 & 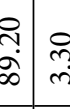 & 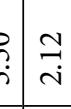 & $\begin{array}{l}\tilde{O} \\
\text { in } \\
\text { in }\end{array}$ & 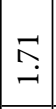 & $\vec{\sim}$ & $\begin{array}{l}\overrightarrow{0} \\
\dot{0}\end{array}$ & \\
\hline $0^{\infty} \overbrace{}^{-1}$ & 7 & & ft. & $\stackrel{\substack{t \\
i}}{i}$ & $\begin{array}{l}\stackrel{0}{0} \\
\dot{m}\end{array}$ & & & 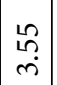 & 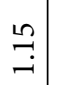 & $\begin{array}{l}\stackrel{\partial}{\circ} \\
\text { in }\end{array}$ & $\begin{array}{l}\vec{b} \\
- \\
-\end{array}$ & & $\begin{array}{l}\infty \\
\infty \\
\dot{n}\end{array}$ & $\begin{array}{l}\stackrel{2}{0} \\
\stackrel{0}{0}\end{array}$ & 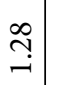 & \begin{tabular}{c|c}
$\underset{N}{N}$ & $\vdots$
\end{tabular} & 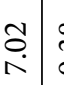 & $\begin{array}{c}\infty \\
\stackrel{\infty}{a} \\
a\end{array}$ & \begin{tabular}{c|c}
$\overrightarrow{1}$ & 2 \\
0 & 0
\end{tabular} & 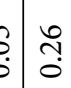 & $\tilde{\tilde{Z}}$ & $\begin{array}{l}\infty \\
0 \\
0\end{array}$ & 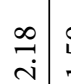 & & \\
\hline
\end{tabular}

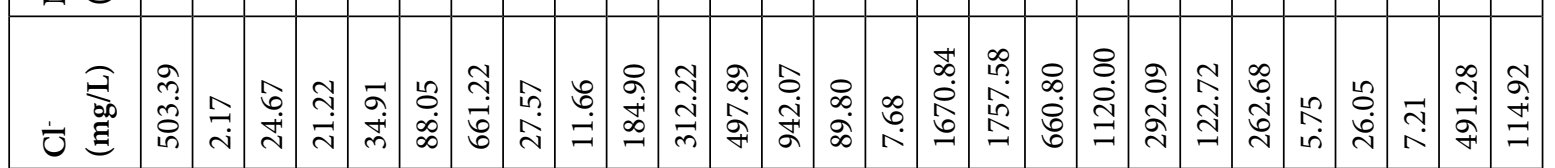

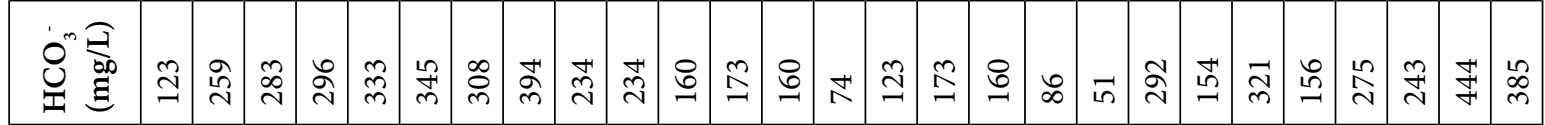

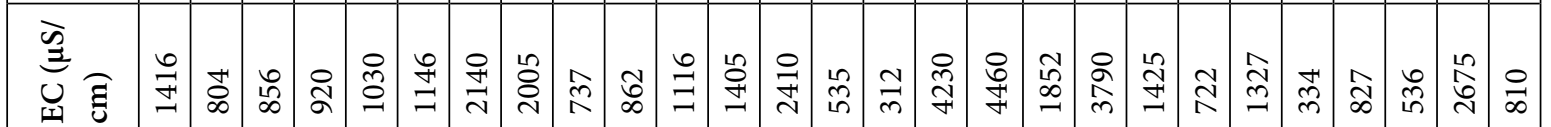

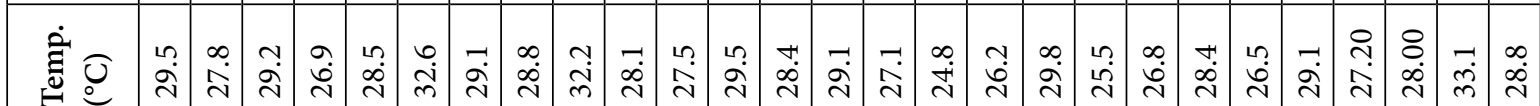

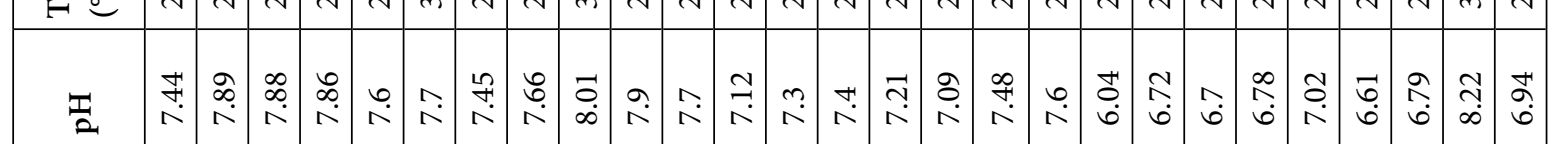

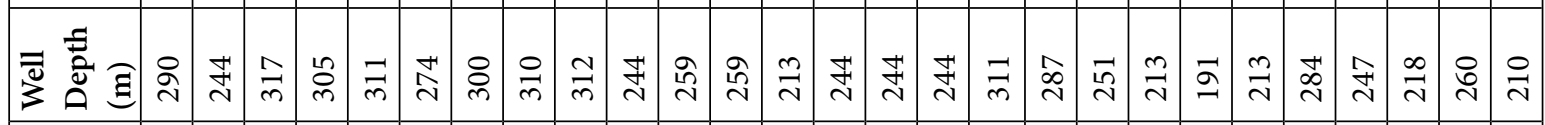

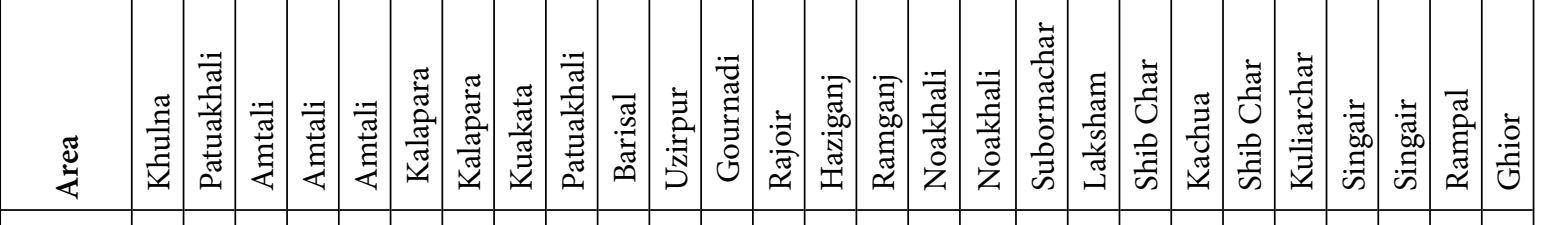

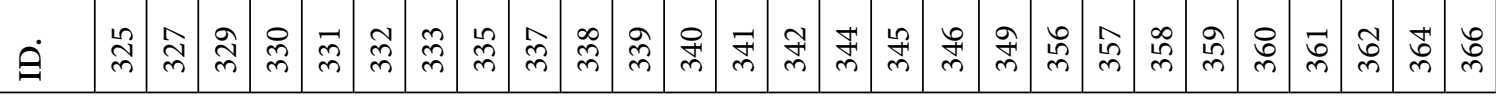


Table 2. Summery statistics of major ion chemistry

\begin{tabular}{|c|c|c|c|c|c|c|c|c|c|}
\hline & $\begin{array}{l}\text { Collected } \\
\text { water } \\
\text { samples }\end{array}$ & $\begin{array}{l}\mathrm{Na}^{+} \\
(\mathrm{mg} / \mathrm{L})\end{array}$ & $\begin{array}{l}\mathrm{Ca}^{2+} \\
(\mathrm{mg} / \mathrm{L})\end{array}$ & $\begin{array}{l}\mathrm{Mg}^{2+} \\
(\mathrm{mg} / \mathrm{L})\end{array}$ & $\begin{array}{l}\mathrm{K}^{+} \\
(\mathrm{mg} / \mathrm{L})\end{array}$ & $\begin{array}{l}\mathrm{HCO}_{3}^{-} \\
(\mathrm{mg} / \mathrm{L})\end{array}$ & $\begin{array}{l}\mathrm{Cl}^{-} \\
(\mathrm{mg} / \mathrm{L})\end{array}$ & $\begin{array}{l}\mathrm{SO}_{4}^{2-} \\
(\mathrm{mg} / \mathrm{L})\end{array}$ & $\begin{array}{l}\mathrm{NO}_{3}^{-} \\
(\mathrm{mg} / \mathrm{L})\end{array}$ \\
\hline \multicolumn{10}{|c|}{ Shallow groundwater } \\
\hline Min. & \multirow{3}{*}{202} & 1.20 & 1.30 & 0.02 & 0.00 & 12.00 & 0.04 & 0.01 & 0.00 \\
\hline Max. & & 2456.60 & 188.84 & 151.25 & 100.31 & 1186.00 & 3420.27 & 321.80 & 159.49 \\
\hline Avg. & & 75.50 & 38.80 & 21.89 & 8.21 & 172.32 & 100.18 & 14.49 & 8.08 \\
\hline \multicolumn{10}{|c|}{ Intermediate depth groundwater } \\
\hline Min. & \multirow{3}{*}{26} & 3.95 & 1.34 & 5.24 & 0.88 & 55 & 0.80 & 0 & 0 \\
\hline Max. & & 268.42 & 87.36 & 40.88 & 56.55 & 394 & 491 & 19.89 & 40.58 \\
\hline Avg. & & 46.69 & 35.07 & 16.31 & 6.16 & 173.39 & 34.71 & 2.49 & 5.15 \\
\hline \multicolumn{10}{|c|}{ Deep groundwater } \\
\hline Min. & \multirow{3}{*}{100} & 5.18 & 3.32 & 0.9 & 0.71 & 37 & 1.59 & 0.01 & 0.01 \\
\hline Max. & & 1063.3 & 240.85 & 434.5 & 54.91 & 449 & 1757.58 & 180.04 & 41.71 \\
\hline Avg. & & 212.33 & 56.22 & 38.43 & 12.65 & 198.17 & 322.45 & 8.52 & 4.28 \\
\hline
\end{tabular}

Table 3. Average ionic composition of major hydrochemical species

\begin{tabular}{|c|c|c|c|c|c|}
\hline Groundwater group & Water type & $\mathrm{Cl}-(\mathrm{mg} / \mathrm{L})$ & HCO3- (mg/L) & $\mathrm{Na}+(\mathrm{Mg} / \mathrm{L})$ & $\mathrm{Ca} 2+(\mathrm{mg} / \mathrm{L})$ \\
\hline \multicolumn{6}{|l|}{ Shallow groundwater } \\
\hline Group-1 & $\mathrm{Ca}-\mathrm{Mg}-\mathrm{HCO}_{3}$ & 9.9 & 215.0 & 13.8 & 51.7 \\
\hline Group-2 & $\mathrm{Na}-\mathrm{Ca}-\mathrm{Mg}-\mathrm{HCO}_{3}$ & 12.5 & 132.9 & 42.5 & 21.8 \\
\hline Group-3 & $\mathrm{Na}-\mathrm{Cl}$ & 910.5 & 304.0 & 632.0 & 43.5 \\
\hline Group-4 & $\mathrm{Ca}-\mathrm{Mg}-\mathrm{Na}-\mathrm{HCO}_{3}-\mathrm{Cl}$ & 73.0 & 148.0 & 24.0 & 42.0 \\
\hline \multicolumn{6}{|c|}{ Intermediate depth groundwater } \\
\hline Group-1 & $\mathrm{Ca}-\mathrm{Mg}-\mathrm{HCO}_{3}$ & 5.6 & 203 & 18.2 & 45.5 \\
\hline Group-2 & $\mathrm{Na}-\mathrm{Ca}-\mathrm{Mg}-\mathrm{HCO}_{3}$ & 3.7 & 133.0 & 67.5 & 23.7 \\
\hline Group-3 & $\mathrm{Na}-\mathrm{Cl}$ & 491.0 & 197.0 & 268.0 & 78.9 \\
\hline Group-4 & $\mathrm{Ca}-\mathrm{Mg}-\mathrm{Na}-\mathrm{HCO}_{3}-\mathrm{Cl}$ & 54.0 & 173.0 & 47.5 & 25.3 \\
\hline \multicolumn{6}{|l|}{ Deep groundwater } \\
\hline Group-1 & $\mathrm{Ca}-\mathrm{Mg}-\mathrm{HCO}_{3}$ & 12.08 & 178.0 & 29.0 & 35.3 \\
\hline Group-3 & $\mathrm{Na}-\mathrm{Cl}$ & 460.05 & 145.5 & 370.0 & 41.0 \\
\hline Group-4 & $\mathrm{Ca}-\mathrm{Mg}-\mathrm{Na}-\mathrm{HCO}_{3}-\mathrm{Cl}$ & 160.0 & 210.5 & 71.0 & 49.0 \\
\hline Group-5 & $\mathrm{Na}-\mathrm{Ca}-\mathrm{Mg}-\mathrm{Cl}$ & 485.0 & 173.0 & 241.0 & 84.0 \\
\hline Group-6 & $\mathrm{Na}-\mathrm{HCO}_{3}$ & 32.0 & 296.0 & 228.0 & 11.0 \\
\hline
\end{tabular}

\subsection{Major Ion Chemistry}

Summery statistics of groundwater major ion chemistry are given in Table 2. The average concentrations of $\mathrm{Na}^{+}$, $\mathrm{Ca}^{2+}$ and $\mathrm{Cl}^{-}$in deep groundwater display a clear difference with shallow and intermediate depth groundwater (Table 2). The trend of major cation concentrations in shallow and deep groundwaters are $\mathrm{Na}^{+}>\mathrm{Ca}^{2+}>\mathrm{Mg}^{2+}>\mathrm{K}^{+}$and for intermediate depth groundwater is $\mathrm{Ca}^{2+}>\mathrm{Na}^{+}>\mathrm{Mg}^{2+}>\mathrm{K}^{+}$. The anionic trend of shallow and intermediate depth groundwaters is $\mathrm{HCO}_{3}>\mathrm{Cl}^{>}>\mathrm{SO}_{4}{ }^{2-}>\mathrm{NO}_{3}^{-}$and deep groundwater is $\mathrm{Cl}^{-}>\mathrm{HCO}_{3}>\mathrm{SO}_{4}{ }^{2-}>\mathrm{NO}_{3}{ }^{-}$. 


\subsection{Hydrochemical Grouping}

Piper plots (Figure 3) for shallow, intermediate and deep groundwater are classified into six major groups, namely Group-1: $\mathrm{Ca}-\mathrm{Mg}-\mathrm{HCO}_{3}$, Group-2: $\mathrm{Na}-\mathrm{Ca}-\mathrm{Mg}-\mathrm{HCO}_{3}$, Group-3: $\mathrm{Na}-\mathrm{Cl}$, Group-4: $\mathrm{Ca}-\mathrm{Mg}-\mathrm{Na}-\mathrm{HCO}_{3}-\mathrm{Cl}$, Group5: $\mathrm{Na}-\mathrm{Ca}-\mathrm{Mg}-\mathrm{Cl}$ and Group-6: $\mathrm{Na}-\mathrm{HCO}_{3}$. The spatial distribution of groundwater hydrochemical species at different depths are show in Figure 3. The relevant chemical parameters for the shallow, intermediate and deep groundwater groups are depicted in Table 3. Figure 3a illustrates that the shallow groundwater is dominantly of $\mathrm{Ca}-\mathrm{Mg}-\mathrm{HCO}_{3}$ type low mineralized water characterizing the chemical composition of rainfall and major river water in Bangladesh, which indicates the initial source of water recharging into the aquifer systems. This type of water (Group-1) is distributed in most sites of the study area (Figure 4a) indicating preferential recharge area. Group-2 shallow groundwater is observed in the northern-eastern site of the study area (Figure. 3a), which shows slightly increase of $\mathrm{Na}^{+}$concentration with respect to $\mathrm{Ca}^{2+}$ and $\mathrm{Mg}^{2+}$. The increase in $\mathrm{Na}^{+}$exchange for $\mathrm{Ca}^{2+}$ and $\mathrm{Mg}^{2+}$ suggest softening process, which may indicate rapid recharge and/or much more water-rock interactions along the flow paths. Group-3 water shown in the central and north-western site (Figure $4 \mathrm{a}$ ) are characterized by $\mathrm{SO}_{4}{ }^{2-}$ and $\mathrm{NO}_{3}^{-}$rich mixed water, which may be from anthropogenic sources. In contrast, the $\mathrm{Na}-\mathrm{Cl}$ type water from the coastal area is characterized by high concentrations of chloride, which is possibly due to mixing with seawater ${ }^{17}$.

The intermediate depth groundwater is also dominated in $\mathrm{Ca}-\mathrm{Mg}-\mathrm{HCO}_{3}$ type water (Figure $3 \mathrm{~b}$ ) and surprisingly in spatial distribution (Figure $4 \mathrm{~b}$ ) the intermediate depth groundwater aquifer with water types $\mathrm{Ca}-\mathrm{Mg}-\mathrm{HCO}_{3}$, $\mathrm{Na}-\mathrm{Ca}-\mathrm{Mg}-\mathrm{HCO}_{3}$ and $\mathrm{Na}-\mathrm{Cl}$ respectively are underlain by the similar type water in shallow groundwater aquifers (Figure 4a). This phenomenon indicates possible connectivity between shallow and intermediate depth aquifers as well as rapid recharge to the intermediate aquifers without changing the chemical characteristics of recharging water. The intermediate depth groundwaters are also affected by softening process giving rise to $\mathrm{Na}-\mathrm{Ca}-\mathrm{Mg}-\mathrm{HCO}_{3}$ type water adding more $\mathrm{Na}^{+}$in groundwater exchanged for $\mathrm{Ca}^{2+}$ and $\mathrm{Mg}^{2+}$. In the coastal region, the intermediate depth groundwaters are characterized by $\mathrm{Na}-\mathrm{Cl}$ chloride type saline water with an average $\mathrm{Cl}^{-}$concentration of 491 $\mathrm{mg} / \mathrm{l}$ (nearly $3 \%$ salinity). Similar to shallow groundwater, the intermediate groundwater is also affected by sea spry or mixed with seawater ${ }^{17}$.

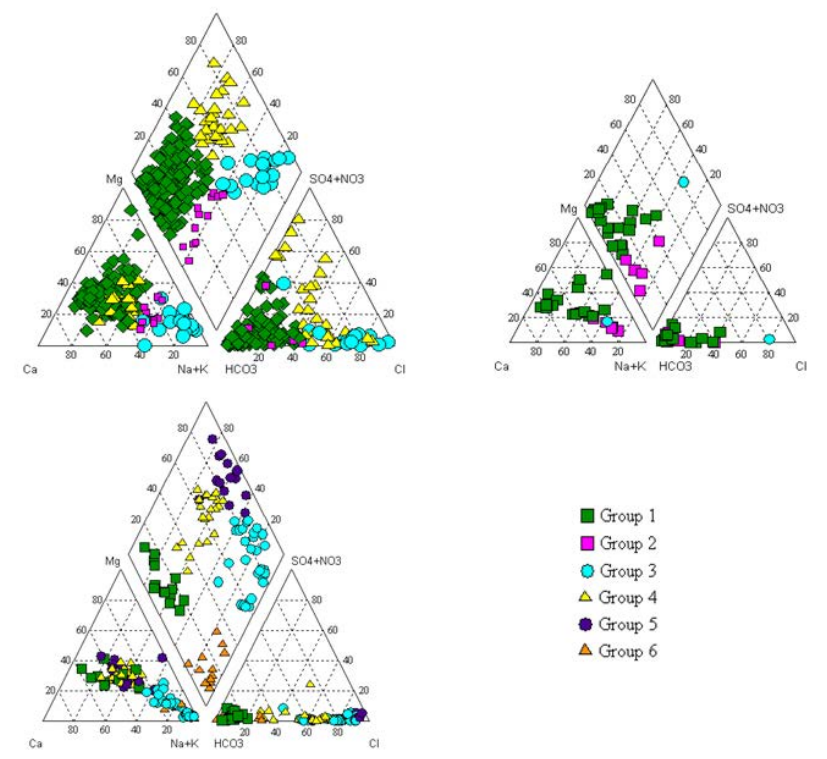

Figure 3. Piper plots showing the major ions composition of groundwater: (a) shallow well $(<70 \mathrm{~m})$, (b) intermediate well $(70-180 \mathrm{~m})$ and $(\mathrm{c})$ deep well $(>180 \mathrm{~m})$. Based on this diagram, groundwaters are classified into six different groups, which are: Group-1 (Ca-Mg- $\left.\mathrm{HCO}_{3}\right)$, Group-2 (Na-Ca-Mg$\mathrm{HCO}_{3}$ ), Group-3 (Na-Cl), Group-4 (Ca-Mg-Na- $\left.\mathrm{HCO}_{3}-\mathrm{Cl}\right)$, Group-5 (Na-Ca-Mg- $\left.\mathrm{HCO}_{3}-\mathrm{Cl}\right)$ and Group-6 $\left(\mathrm{Na}-\mathrm{HCO}_{3}\right)$.

The Piper plot for deep groundwater (Figure 3c) shows distinct groundwater types both in compositions and in spatial distributions (Figure 4c). $\mathrm{Ca}-\mathrm{Mg}-\mathrm{HCO}_{3}$ type water is observed in deep wells lying in the northern site of the study area. The $\mathrm{Na}-\mathrm{Cl}$ and $\mathrm{Na}-\mathrm{Ca}-\mathrm{Mg}-\mathrm{Cl}$ type deep groundwaters are restricted in the coastal region (Figure 4c) having 0.9 to $6 \%$ salinity. This brackish high chloride content water probably represents relic seawater trapped in sediments during deposition under marine regressive conditions that have later undergone certain modifications (e.g., cation exchange, diluting by mixing with fresh meteoric water) during its period of confinement. Similar type of deep saline groundwater was also observed by ${ }^{18}$ in West Bengal, India. The $\mathrm{Ca}-\mathrm{Mg}-\mathrm{Na}-\mathrm{HCO}_{3}-\mathrm{Cl}$ (Group4) type deep groundwater shows increase in chloride and bicarbonate concentrations. The gradual increase in deep groundwater $\mathrm{Cl}^{-}$and $\mathrm{Na}^{+}$concentrations thus suggest groundwater flow from the area of Group-1 type water towards the mixed type Group-4 area (Figure 4c).

The $\mathrm{Na}-\mathrm{HCO}_{3}$ type low chloride content (average $32 \mathrm{mg} / \mathrm{L}$ ) groundwaters are observed in the coastal deep 
aquifers. It indicates that the $\mathrm{Ca}-\mathrm{HCO}_{3}$ groundwaters progressively evolve into the $\mathrm{Na}-\mathrm{HCO}_{3}$ type water at greater depth and the concentrations of chemical constituents in the water increase with prolonged water-rock interactions ${ }^{19}$. The presence of $\mathrm{Na}-\mathrm{HCO}_{3}$ type water in deep aquifers usually represents the end member of the groundwater flow system ${ }^{20}$. The $\mathrm{Na}-\mathrm{HCO}_{3}$ type deep groundwater observed in the coastal aquifers of Bangladesh are of stagnant water, which probably reflects the effect of incomplete flushing in a buried estuary aligned on an old course of the Ganges and/or Brahmaputra rivers when they flow directly south from their present confluence ${ }^{21}$.
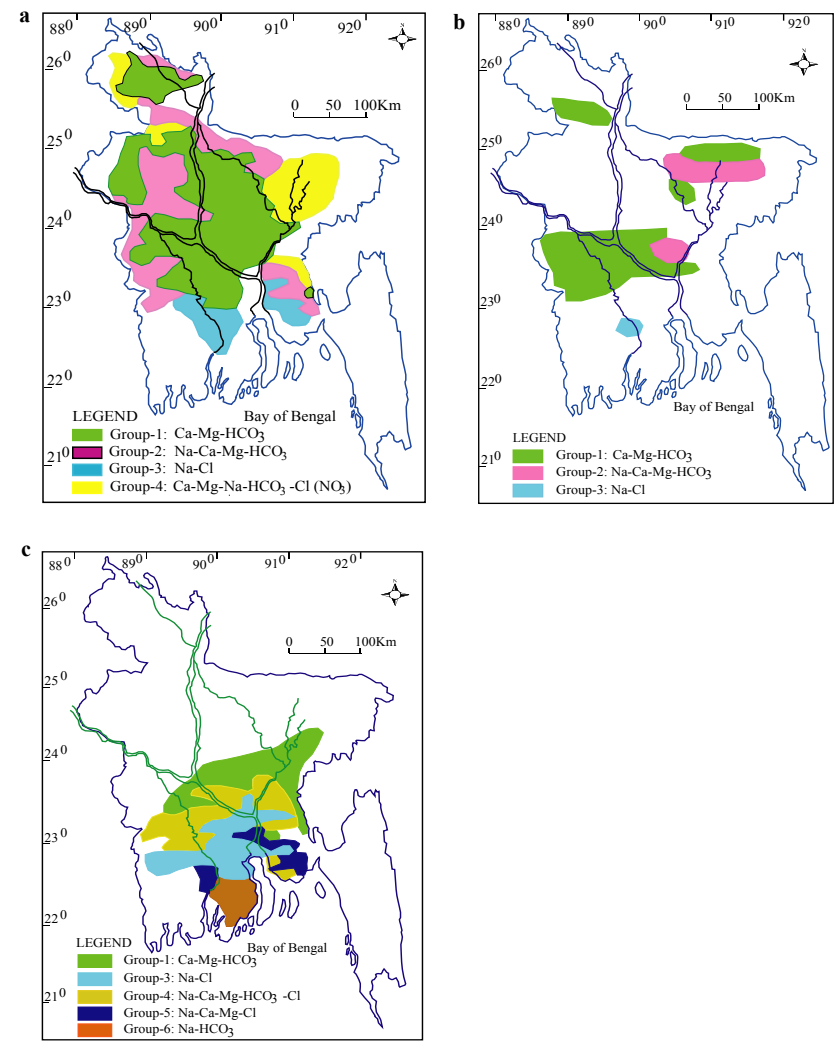

Figure 4. Plots showing the spatial distribution of groundwater hydrochemical species at different depths (a) shallow well (<70 m), (b) intermediate well (70-180 m) and (c) deep well $(>180 \mathrm{~m})$.

\subsection{Stiff Diagram}

Stiff diagrams are widely used to infer the trend of groundwater mineralization along the groundwater flow paths in spatial distribution. The spatial distribution of shallow groundwater chemical types represented in the Stiff diagrams (Figure 5a) denotes the generalized progressive increase of shallow groundwater mineralization from north to south. The increase in sodium and chloride concentration is prominent in the coastal region, which is affected by mixing with seawater. Stiff diagrams (Figure $5 b)$ for intermediate depth groundwater show low mineralized water, which indicates rapid recharge into the aquifers without any significant chemical change in the initial recharging water (Group-1 type) as well as indicates low residence times for water-rock interactions or relatively short flow paths. The spatial distribution of deep groundwater Stiff diagrams (Figure 5c) shows notably high-mineralized water than those of shallow and intermediate groundwater. The chemical pattern of deep groundwater progressively increases from north to south. Following this direction, a gradual decrease in calcium and increase in sodium is noticeable. The chloride concentration increases in accordance with the pattern, which can be specified as flow directions.
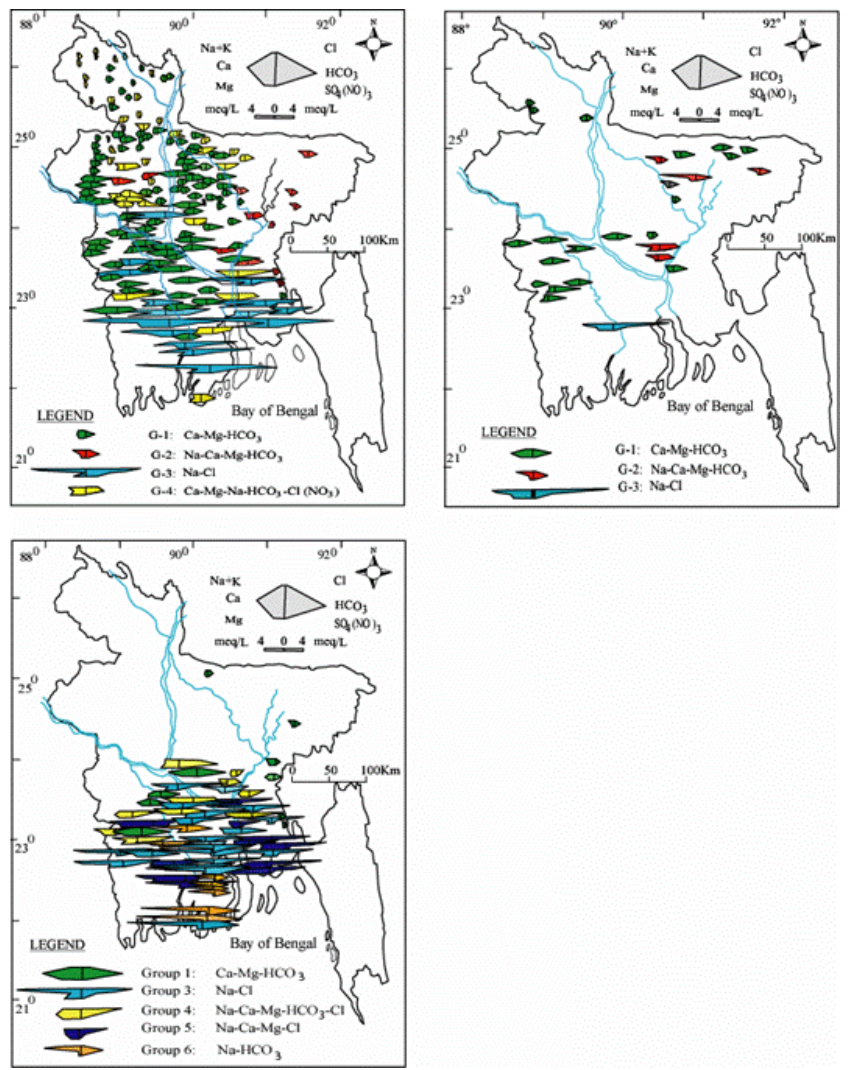

Figure 5. Stiff diagram showing (a) shallow, (b) intermediate and (c) deep groundwater hydrochemical types distributed over the study area.

\subsection{Carbonates Dissolution}

Bicarbonate in Bengal Delta groundwaters may derive mainly from the soil zone $\mathrm{CO}_{2}$ and weathering of parent 
minerals. The soil zone in the subsurface contains elevated $\mathrm{CO}_{2}$ pressure (produced by decay of organic matter and root respiration), which in turn combines with rainwater to form bicarbonate ${ }^{22}$ following the reactions given below:

$\mathrm{CO}_{2}+\mathrm{H}_{2} \mathrm{O} \rightarrow \mathrm{H}_{2} \mathrm{CO}_{3}$
$\mathrm{H}_{2} \mathrm{CO}_{3} \rightarrow \mathrm{H}^{+}+\mathrm{HCO}_{3}^{-}$

Bicarbonate may also be derived from the dissolution of carbonates minerals (calcite and dolomite) by the carbonic acid according to:

$\mathrm{CaCO}_{3}+\mathrm{H}_{2} \mathrm{CO}_{3} \rightarrow \mathrm{Ca}^{2+}+2 \mathrm{HCO}_{3}^{-}$

-(iii)

(calcite)

$\mathrm{CaMg}\left(\mathrm{CO}_{3}\right)_{2}+2 \mathrm{H}_{2} \mathrm{CO}_{3} \rightarrow \mathrm{Ca}^{2+}+\mathrm{Mg}^{2+}+4 \mathrm{HCO}_{3}^{-}-\cdot-\cdot-\cdot$

(iv)

(dolomite)

If $\mathrm{Ca}^{2+}$ and $\mathrm{Mg}^{2+}$ in groundwater may come from the dissolution of calcite and dolomite according to the equations (iii) and (iv) respectively, there would be straight positive correlation between $\mathrm{Ca}^{2+}$ and $\mathrm{HCO}_{3}{ }^{-}$and, $\mathrm{Mg}^{2+}$ and $\mathrm{HCO}_{3}^{-}$. A bi-variant plot (Figure 6a) of $\mathrm{Ca}^{2+}$ versus $\mathrm{HCO}_{3}^{-}$ shows poor correlation $\left(r^{2}=0.30\right)$ for shallow, intermediate $\left(r^{2}=0.03\right.$, regression line not shown in Figure 6a) and deep groundwater $\left(\mathrm{r}^{2}=0.009\right.$, regression line not shown in Figure $6 a)$. It is evident that $\mathrm{Ca}^{2+}$ in all observed groundwaters may not come from calcite dissolution. Besides ${ }^{23}$ observed poor amount of calcite (average $0.8 \mathrm{wt} \%$ ) in Bengal Delta sediments and thus this minor amount of calcite in host sediments may not be responsible releasing higher concentrations for $\mathrm{Ca}^{2+}$ in Bengal Delta groundwater.
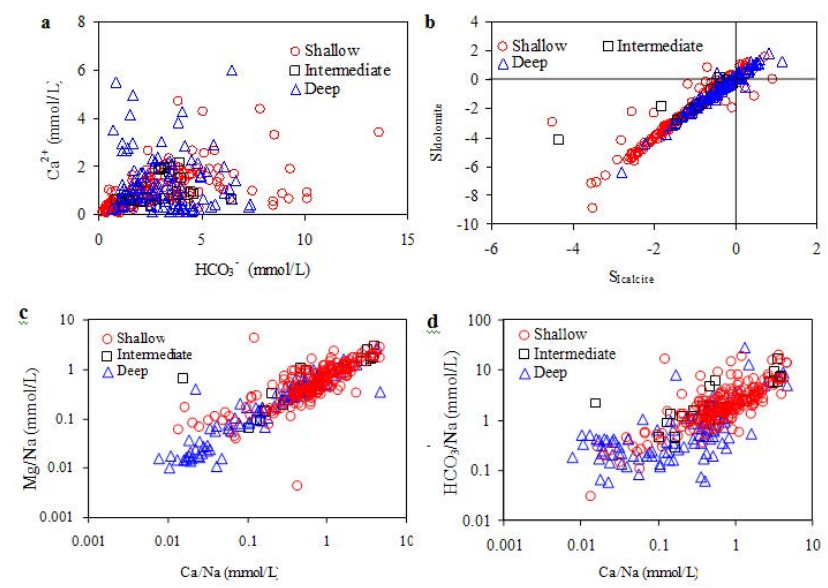

Figure 6. Bivariate plots showing the correlation between (a) $\mathrm{HCO}_{3}{ }^{-}$versus $\mathrm{Ca}^{2+}$, (b) Saturation Index for calcite versus dolomite, (c) $\mathrm{Ca} / \mathrm{Na}$ versus $\mathrm{Mg} / \mathrm{Na}$ and (d) $\mathrm{Ca} / \mathrm{Na}$ versus $\mathrm{HCO}_{3} / \mathrm{Na}$.
Assuming pure water equilibrated with sedimentary calcite and assuming $10^{-3.5} \mathrm{~atm}$ soil $\mathrm{CO}_{2}$ gas in an open system at $25^{\circ} \mathrm{C}$, the geochemical properties mainly saturation index for calcite and dolomite have been simulated, as given in Table 1. The plot of saturation indices of calcite $\left(\mathrm{SI}_{\text {calcite }}\right)$ versus dolomite $\left(\mathrm{SI}_{\text {dolomite }}\right)$ demonstrates that most of the groundwaters are under-saturated with respect to dolomite and calcite (Figure 6b). According to Figure $6 \mathrm{~b}$, about $86 \%$ of the analyzed groundwater samples are under-saturated with calcite and dolomite. It represents that the water comes from an environment where calcite and dolomite are impoverished. It also indicates that in the Bengal Delta groundwater, $\mathrm{Ca}^{2+}$ and $\mathrm{Mg}^{2+}$ partially come from carbonate dissolution.

Surprisingly, only $25 \%$ deep groundwaters (out of 100 nos.) are saturated with calcite and it indicates precipitation of calcium as calcite and/or dolomite.

\subsection{Silicate Weathering}

Weathering reactions of $\mathrm{Ca}$ and $\mathrm{Mg}$-silicates are also responsible releasing $\mathrm{Ca}^{2+}$ and $\mathrm{Mg}^{2+}$ transforming $\mathrm{CO}_{2}$ from the atmosphere to $\mathrm{HCO}_{3}{ }_{3}^{-}$in groundwater as:

$2 \mathrm{NaAlSi}_{3} \mathrm{O}_{8}+2 \mathrm{H}_{2} \mathrm{CO}_{3}+9 \mathrm{H}_{2} \mathrm{O} \rightarrow \mathrm{Al}_{2} \mathrm{Si}_{2} \mathrm{O}_{5}(\mathrm{OH})_{4}+2 \mathrm{Na}^{+}+$ $4 \mathrm{H}_{4} \mathrm{SiO}_{4}+2 \mathrm{HCO}_{3}^{--}---(\mathrm{v})$

(Na-silicate)

$\mathrm{Mg}_{2} \mathrm{SiO}_{4}+4 \mathrm{CO}_{2}+4 \mathrm{H}_{2} \mathrm{O} \rightarrow 2 \mathrm{Mg}^{2+}+4 \mathrm{HCO}_{3}^{-}+\mathrm{H}_{4} \mathrm{SiO}_{4}^{----}$ (Mg-silicate)

Therefore, the Na-normalized ${ }^{24}$ ratios for $\mathrm{Ca}^{2+}$ and $\mathrm{Mg}^{2+}$ might have relationship to each other. Accordingly, in the plot of molar ratios of $\mathrm{Ca} / \mathrm{Na}$ versus $\mathrm{Mg} / \mathrm{Na}$ are shown in a log-log space in Figure 6c, both shallow and deep groundwater show moderate correlation with regressions $\mathrm{r}^{2}=0.54$ and $\mathrm{r}^{2}=0.55$ respectively, whereas intermediate depth groundwater shows higher correlation $\left(r^{2}=0.85\right)$. Recharging waters flowing through carbonates rich aquifer show high $\mathrm{Ca} / \mathrm{Na}$ and $\mathrm{Mg} / \mathrm{Na}$ rations (Figure $6 \mathrm{c}$ ). The end member having lower Na-normalized ratios is that of water draining silicates. The molar $\mathrm{Ca} / \mathrm{Na}$ ratio of average crustal continental rocks is close to $0.6^{25}$, and due to the higher solubility of $\mathrm{Na}$ relative to $\mathrm{Ca}$, lower $\mathrm{Ca} / \mathrm{Na}$ molar ratio are expected in groundwater, which are related to weathering of silicates. In Figure $6 c$, the observed shallow groundwater with high $\mathrm{Ca} / \mathrm{Na}$ molar ratios are being influenced by carbonate dissolution, whereas the intermediate and deep groundwaters are influenced by 
silicate weathering rather than carbonate dissolution. Similarly, the plot (Figure 6d) for $\mathrm{HCO}_{3} / \mathrm{Na}$ and $\mathrm{Ca} / \mathrm{Na}$ molar rations, high molar rations for half of the shallow and intermediate depth groundwaters are an indication of carbonate dissolution, meanwhile low molar ratios of $\mathrm{HCO}_{3} / \mathrm{Na}$ and $\mathrm{Ca} / \mathrm{Na}$ for the deep groundwaters are the indication of silicate weathering.

If groundwater mainly recharged by the recent atmospheric precipitation, its circulation remains active $e^{26}$. This being so, the most likely mechanism that can increase concentrations of $\mathrm{Na}^{+}$and $\mathrm{HCO}_{3}^{-}$in groundwater is the alteration of silicates (like albite) as per the following reaction:

$$
\begin{aligned}
& \mathrm{NaAlSi}_{3} \mathrm{O}_{8}+\mathrm{CO}_{2}(\mathrm{aq})+11 / 2 \mathrm{H}_{2} \mathrm{O} \\
& (\mathrm{OH})_{4}+2 \mathrm{H}_{4} \mathrm{SiO}_{4}+\mathrm{NCO}^{+}+1 / 2 \mathrm{Al}_{2} \mathrm{Si}_{2} \mathrm{O}_{5} \\
& ----(\mathrm{vii})
\end{aligned}
$$

This reaction leads to increase in $\mathrm{Na}^{+}$and $\mathrm{HCO}_{3}{ }^{-}$concentrations consuming $\mathrm{CO}_{2}(\mathrm{aq})$, and thus decreases the partial pressure of carbon dioxide $\left(\mathrm{pCO}_{2}\right)$ and increases $\mathrm{pH}$. The high average $\mathrm{Na}^{+}(212.33 \mathrm{mg} / \mathrm{L})$ content in observed deep groundwater with high $\mathrm{pH}(>7.5)$ values comply with the above-mentioned argument.
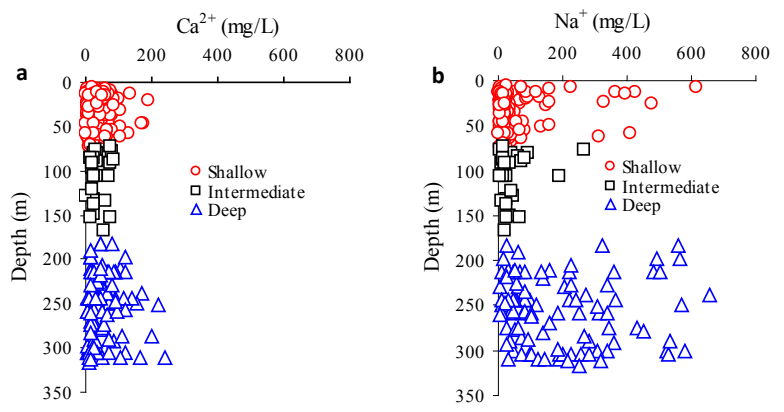

Figure 7. Depth dependence plots showing variations between (a) $\mathrm{Ca}^{2+}$ versus depth and (b) $\mathrm{Na}^{+}$versus depth.

\subsection{Cation Exchange}

Cation exchange reaction is also responsible for $\mathrm{Na}^{+}$ enrichment in groundwater $\mathrm{as}^{2 \mathrm{27}}$ :

$$
1 / 2 \mathrm{Ca}^{2+}+\mathrm{Na}-\mathrm{X} \rightarrow 1 / 2 \mathrm{Ca}-\mathrm{X}_{2}+\mathrm{Na}^{+}
$$

where, $\mathrm{X}$ denotes cation-exchange sites. This reaction explains the increase in $\mathrm{Na}^{+}$concentration without an associated increase in $\mathrm{Cl}^{-}$concentration along the flow path and the clay particles of aquifer exchange calcium against sodium to elevate sodium concentrations ${ }^{26}$. It represents a process whereby a brackish aquifer is flushed with fresh water.
Considering the depth dependence of $\mathrm{Na}^{+}$and $\mathrm{Ca}^{2+}$ (Figure $7 \mathrm{a}, 7 \mathrm{~b}$ ), it is found that both $\mathrm{Ca}^{2+}$ and $\mathrm{Na}^{+}$has shown low concentrations up to the base of the intermediate depth aquifer. Only few shallow groundwater samples show slightly high $\mathrm{Na}^{+}$concentrations. Meanwhile, in deep groundwater $\mathrm{Ca}^{2+}$ concentrations remain nearly unchanged, whereas $\mathrm{Na}^{+}$concentrations become high. In Bengal delta, the $\mathrm{Na}^{+}$concentration increases in deep groundwater aquifers in response to cation exchange for $\mathrm{Ca}^{2+}$.

\subsection{Mixing of Groundwater}

In general, chloride is a conservative component, and evaporation and mixing are considered as the main factors controlling its concentration in groundwater. Solubility of $\mathrm{Na}^{+}$compounds is high, so $\mathrm{Na}^{+}$remains dissolved in water in a very wide range of concentration ${ }^{28}$. In $\mathrm{Na}^{+}$versus $\mathrm{Cl}^{-}$plot, most of the shallow and intermediate depth groundwater lie along the 1:1 evolution line (Figure $8 \mathrm{a}$ and Figure $8 \mathrm{~b}$ ) and it indicates that these water have mainly originated from rainfall and/or flood water maintaining the evolutionary ratio between $\mathrm{Na}^{+}$and $\mathrm{Cl}^{-}(1: 1)$. Nevertheless, the deep groundwater scattered in three groups as samples along the $\mathrm{Na}$-axis, above the seawater line (slope 0.86) and below the seawater line (Figure 8c). Those water scattered close to $\mathrm{Na}$-axis (Y-axis in Figure 8c) are of $\mathrm{Na}-\mathrm{HCO}_{3}$ groundwater observed in coastal deep aquifers and these are of stagnant water having low concentration of chloride ( $<48 \mathrm{mg} / \mathrm{L}$ ). Whereas, the deep groundwaters scattered above the seawater line show $\mathrm{Na}^{+}$excess and $\mathrm{Na}^{+}$excess suggest the presence of deep groundwater flow which gives rise to excess $\mathrm{Na}^{+}$along the flow paths due to cation exchange of $\mathrm{Na}^{+}$for $\mathrm{Ca}^{2+}$ as per the equation (viii). The deep groundwater lies below the seawater line (Figure $8 \mathrm{c}$ ) are mainly of $\mathrm{Na}-\mathrm{Cl}$ and $\mathrm{Na}-\mathrm{Ca}-$ $\mathrm{Mg}-\mathrm{Cl}$ type water with residence time of about 8500 year BP. If it is considered that the deep groundwater scattered below the seawater line is influenced by present seawater, then the process will have followed the equation ${ }^{27}$ given below:

$\mathrm{Na}^{+}+1 / 2 \mathrm{Ca}-\mathrm{X}_{2} \rightarrow \mathrm{Na}-\mathrm{X}+\mathrm{Ca}^{2+}$

Where, $\mathrm{X}$ indicates the soil exchanger.

This reaction will lead to increase in $\mathrm{Ca}^{2+}$ in coastal aquifers having $\mathrm{Ca}-\mathrm{Cl}_{2}$ type water. Surprisingly observed deep groundwater do not show any $\mathrm{Ca}-\mathrm{Cl}_{2}$ type water. Besides, the deep groundwater falls along the seawater line show long residence time ( $\sim 6000$ to 25000 year $\mathrm{BP})^{\frac{12}{}}$, and these are also $\mathrm{Na}-\mathrm{Cl}$ and $\mathrm{Na}-\mathrm{Ca}-\mathrm{Mg}-\mathrm{Cl}$ type 
water. This water may partially mix with remnant seawater maintaining maxing ratios ${ }^{29}$. Thus, it may conclude that the $\mathrm{Na}-\mathrm{Cl}$ and $\mathrm{Na}-\mathrm{Ca}-\mathrm{Mg}-\mathrm{Cl}$ type deep groundwater are not influenced by the present seawater intrusion in the coastal aquifers of Bangladesh. The $\mathrm{Na}-\mathrm{Cl}$ salinity may come from leaching of marine sediments, which are often dominated in coastal aquifers ${ }^{29}$. Alternatively, this brackish deep groundwater is probably of remnant seawater trapped within lower-permeability sediments ${ }^{29}$. Furthermore $\frac{18}{}$ observed similar type brackish connate water pockets in the western site of Bengal Delta, West Bengal, India.
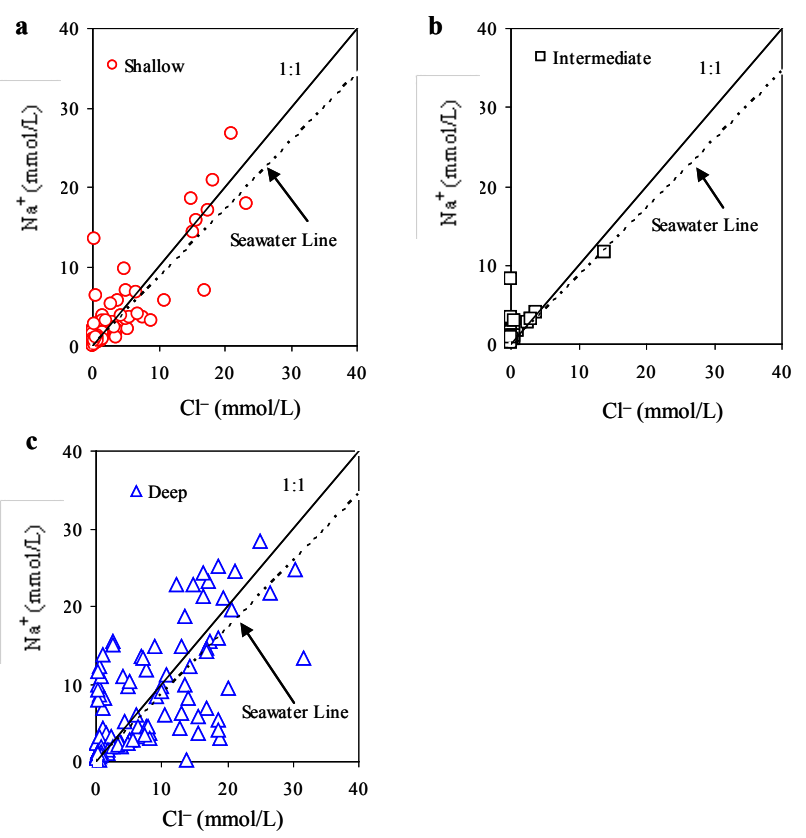

Figure 8. Bivariate plots showing relationship between (a) shallow groundwater $\mathrm{Na}^{+}$versus $\mathrm{Cl}^{-}$, (b) intermediate groundwater $\mathrm{Na}^{+}$versus $\mathrm{Cl}^{-}$and (c) deep groundwater $\mathrm{Na}^{+}$ versus $\mathrm{Cl}^{-}$.

\section{Discussions}

$\mathrm{Ca}-\mathrm{Mg}-\mathrm{HCO}_{3}$ type shallow groundwater (Figure 4a) is widely distributed in most of the study area, which indicates infiltration of modern meteoric water as rain or flood in to the shallow groundwater aquifers. The $\mathrm{Ca}-\mathrm{Mg}-\mathrm{HCO}_{3}$ type groundwater is also available in the intermediate depth aquifers up to the depth $180 \mathrm{~m}$ in the west-central and north-eastern region (Figure $4 \mathrm{~b}$ ) of Bangladesh. It is a clear indication of active recharge to the intermediate aquifers, which may ultimately feed the deeper aquifers. In the west-central and eastern sites of the study area, low mineralized $\mathrm{Ca}-\mathrm{Mg}-\mathrm{HCO}_{3}$ type groundwater is observed at deep aquifers underlying the shallow and intermediate aquifers with similar water type $\left(\mathrm{Ca}-\mathrm{Mg}-\mathrm{HCO}_{3}\right)$ (Figure 4c). This implies active recharge to the deep aquifers from the west-central and eastern mountainous regions of the study area.

In shallow or local flow systems, the flow path is relatively short and thus shallow groundwater does not show distinct hydrochemical changes, i.e., change in water type. The presence of $\mathrm{Na}-\mathrm{Ca}-\mathrm{Mg}-\mathrm{HCO}_{3}$ water in intermediate aquifers (Figure 5b) is mainly evolved from $\mathrm{Ca}-\mathrm{Mg}-\mathrm{HCO}_{3}$ water. It may indicate vertical groundwater and/or local groundwater flow within both the shallow and intermediate depth groundwaters with low water-rock interaction, i.e., short residence time. Along the flow path, significant change in concentration of major cations takes place ${ }^{28}$. The $\mathrm{Ca}-\mathrm{Mg}-\mathrm{HCO}_{3}$ type deep groundwater observed in the west-central, middle and eastern (recharging area) part of the study area corresponds to the beginning of the flow path of the deep flow system (Figure 5c). As deep groundwater flows, the initial $\mathrm{Ca}-\mathrm{Mg}-\mathrm{HCO}_{3}$ water evolves into $\mathrm{Na}-\mathrm{Cl}$ type water mixing with connate paleoseawater with low concentrations of $\mathrm{Ca}^{2+}$ and $\mathrm{Mg}^{2+}$, and the high $\mathrm{Na}^{+}$concentrations in the central and southern coastal region of Bangladesh.

As per Figure $5 \mathrm{a}$ and Figure $5 \mathrm{c}$, in the study area groundwater flows from the $\mathrm{Ca}-\mathrm{Mg}-\mathrm{HCO}_{3}$ rich unconfined to $\mathrm{Na}-\mathrm{Cl}$ rich confined (deep) aquifer and results in the evolution of $\mathrm{Ca}-\mathrm{Mg}-\mathrm{HCO}_{3}$ groundwater to $\mathrm{Na}-\mathrm{Cl}$ type water. Hydrochemical data plot (Figure 8c) suggests that the excess $\mathrm{Na}^{+}$is due to cation exchange of $\mathrm{Na}^{+}$for $\mathrm{Ca}^{2+}$. Besides, the excess $\mathrm{Na}^{+}$in the deep flow system at the end of the flow path indicates an additional $\mathrm{Na}^{+}$ source, which is attributed to silicate weathering (Figure $6 c, 6 d)$. Meanwhile, additional $\mathrm{Na}^{+}$in the deep groundwater may come from clay deposited from the marine episodes which acts as a long-term source of $\mathrm{Na}^{+}$and $\mathrm{Cl}^{-}$ to the underlying aquifer ${ }^{30}$.

Considering the depth dependence of $\mathrm{Na}^{+}$and $\mathrm{Ca}^{2+}$, it is found that the average $\mathrm{Ca}^{2+}$ concentration is low (38.14 $\mathrm{mg} / \mathrm{L}$ ) in shallow groundwater, while $\mathrm{Na}^{+}$concentrations are more than two times higher $(74.75 \mathrm{mg} / \mathrm{L})$ than that of $\mathrm{Ca}^{2+}$ concentrations (Figure 7a, 7b). In Figure 7b, the relatively high $\mathrm{Na}^{+}$concentrations are observed in the coastal shallow wells, which is due to mix with seawater (Figure 8a). In intermediate depth aquifers, the composition of $\mathrm{Ca}^{2+}$ and $\mathrm{Na}^{+}$become homogeneous and the concentra- 
tions of dissolved $\mathrm{Ca}^{2+}(37.25 \mathrm{mg} / \mathrm{L})$ and $\mathrm{Na}^{+}(46.66 \mathrm{mg} / \mathrm{L})$ are low (Figure $7 \mathrm{a}, \mathrm{b}$ ). Going downward, it is found that $\mathrm{Ca}^{2+}$ concentrations remain unchanged (Figure 7a), while $\mathrm{Na}^{+}$concentrations become high in deep aquifers (Figure $7 b)$. It implies that the $\mathrm{Na}^{+}$concentration in groundwater increases in the deep aquifers in response to ion exchange of $\mathrm{Na}^{+}$for $\mathrm{Ca}^{2+}$ and this phenomenon is also supported by the presence of clay minerals in the deep aquifer materials ${ }^{\underline{30}}$. Appelo and Postma ${ }^{27}$ explain that mineral dissolution and precipitation is a well-known category of chemical reactions that can have an important impact on solute concentrations.

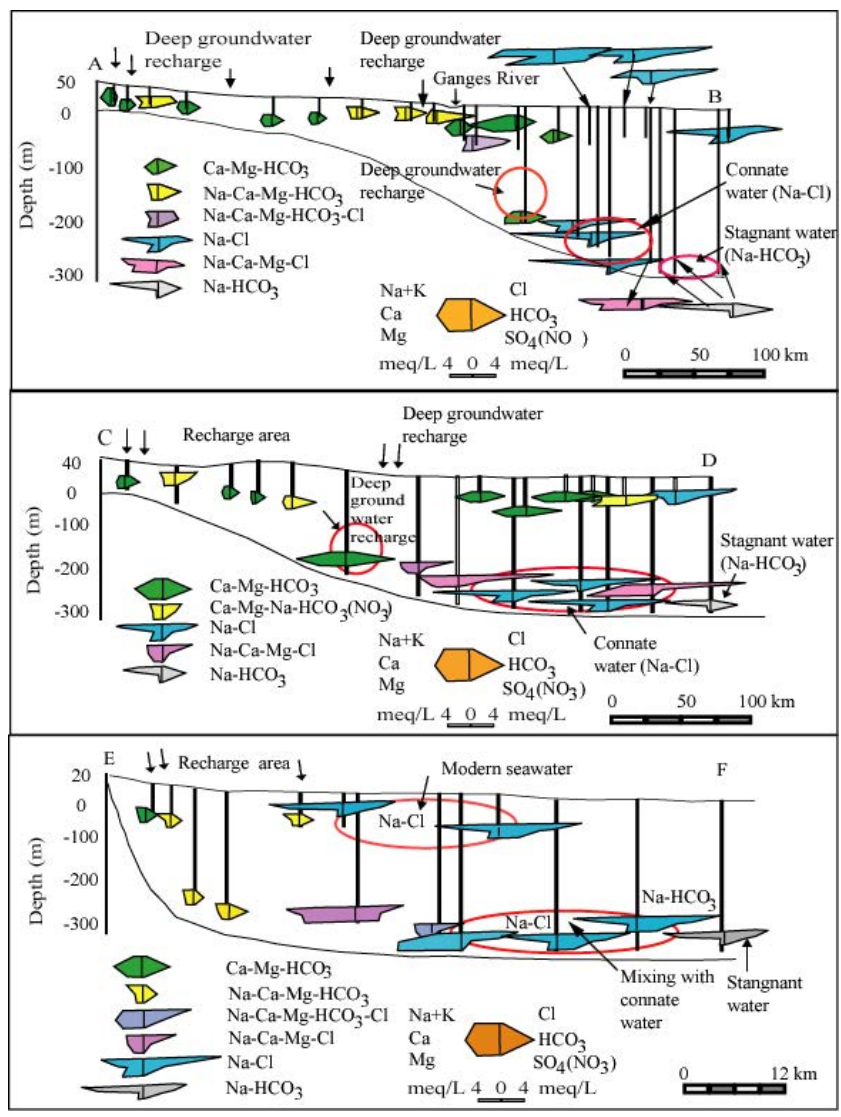

Figure 9. Distribution of chemical species in groundwater at different depths along cross-section lines (a) A-B, (b) C-D and (c) E-F shown in Figure 1.

In present study area, according to the hydrochemical cross-sections (Figure 9), the observed groundwater samples show significant change in major cation $\left(\mathrm{Ca}^{2+}\right.$ and $\mathrm{Na}^{+}$) concentrations. The shallow wells with $\mathrm{Ca}^{-}$ $\mathrm{Mg}-\mathrm{HCO}_{3}$ type groundwater situated in the northern, west-central and eastern site of the study area correspond to the beginning of the shallow flow system and thus ulti- mately feeds both the intermediate and deep groundwater systems (Figure 9a, b, c). Meanwhile, deep groundwater recharges in the northern (Figure 9a, b), central (Figure $9 \mathrm{a}, \mathrm{b}$ ) and eastern (Figure 9c) sites of the study area with $\mathrm{Ca}-\mathrm{Mg}-\mathrm{HCO}_{3}$ water.

Above-mentioned arguments comply with the statement of Kinniburgh and Smedley ${ }^{12}$ and they state that there has been incision of the main Brahmaputra valley along with basal fan-delta sediments, which are deposited between uplifted Pleistocene Residual deposits (Figure 1). These coarse-grained sediments thin and pinch out south (Figure 2) of the Continental Slope (Hinge Zone) (Figure 2) and pass laterally into sandy deltaic deposits within the subsiding Faridpur Trough (Bengal Foredeep) (Figure 2). This coarse-grained layer would be the possible source of recharge through which low mineralized $\mathrm{Ca}-\mathrm{Mg}-\mathrm{HCO}_{3}$ type water enters in to the deep aquifer system. Furthermore, deep groundwater moves towards south (Figure 9a, b) or south-west (Figure 9c) and mixes with $\mathrm{Na}-\mathrm{Cl}$ type connate water with excess $\mathrm{Na}^{+}$. The deep $\mathrm{Na}-\mathrm{HCO}_{3}$ type water along the coastal belt (Figure 9a, b) probably reflects an incomplete flushing in a buried estu$\operatorname{ary} \underline{21}$.

In the recharge area, the $\mathrm{Ca}^{2+}$ concentration is decreasing from the shallow to the deep layers along the flow paths (Figure 9a, b, c). In infiltrating water, the source of $\mathrm{Ca}^{2+}$ is the dissolution of carbonate minerals, which is controlled by local partial pressure of $\mathrm{CO}_{2}$ and the $\mathrm{CO}_{2}$ originates from the transformation of organics. In shallow groundwater, differences in concentrations of $\mathrm{Ca}^{2+}$ reflect different local partial pressures of $\mathrm{CO}_{2}$. As groundwater moving downward, $\mathrm{CO}_{2}$ partial pressure becomes homogeneous. In the discharge areas, changing of $\mathrm{Na}^{+}$is the mirror image of $\mathrm{Ca}^{2+}$ due to ion exchange. The excess $\mathrm{Na}^{+}$in the deep flow system at the end of the flow path indicates an additional $\mathrm{Na}^{+}$source, which is attributed to weathering of $\mathrm{Na}^{+}$feldspars. As per the hydrochemical sections (Figure 9a, b, c), the possible geochemical processes involved within Bengal Delta aquifers are silicate weathering with partial carbonate dissolution in the shallow aquifers giving rise to $\mathrm{Ca}-\mathrm{Mg}-\mathrm{HCO}_{3}$ and $\mathrm{Na}-\mathrm{Ca}-\mathrm{Mg}-\mathrm{HCO}_{3}$ type waters; $\mathrm{Na}^{+}$for $\mathrm{Ca}^{2+}$ ion exchange and finally mixing with deep $\mathrm{Na}-\mathrm{Cl}$ type connate water possibly originated from the diffusion of marine clay ${ }^{30}$. The $\mathrm{Na}-\mathrm{Cl}$ rich deep saline waters are probably trapped within lower-permeability sediments reflecting incomplete mixing and flushing ${ }^{31}$. Furthermore, DPHE ${ }^{32}$ states that the shallow aquifer in the west-central region (Figure 
1) is very thick where there is no deep aquifer up to about $250 \mathrm{~m}$ depth. Kinniburgh and Smedley $\underline{13}$ also affirm that the south-central region (Figure 1) of Bangladesh is underlain by stacked sequences of coarse sands and gravels between $50-240 \mathrm{~m}$ below the ground surface. These coarse sediments appear to have been deposited in the former main channel of the Ganges River. It is evident that the coarse grained thick aquifers in the west-central region of Bangladesh facilitate recharge in to the deep aquifers having similar groundwater type $\left(\mathrm{Ca}-\mathrm{Mg}-\mathrm{HCO}_{3}\right)$ both in shallow and deep groundwater (Figure 9a) and it is a clear indication of deep groundwater recharge in the west-central region of Bangladesh.

Fining-upward sequences of gravels and coarse to medium sands with basal conglomerate occur within the Residual deposits (Figure 1) of the Brahmaputra main channel beneath the central region of Bangladesh (Figure 9b), which pinch out south of the Continental Slope (Hinge Zone) (Figure 2) and pass laterally (Figure 2 ) into the sandy deltaic deposits $\frac{13}{}$. In the central region of Bangladesh (Figure 1), recharge to the deep aquifers (Figure 9b) is due to the presence of aforementioned coarse grained sequence with $\mathrm{Ca}-\mathrm{Mg}-\mathrm{HCO}_{3}$ type low mineralized water. Meanwhile, the deep aquifers may recharge from the overlying shallow aquifers through stratigraphic short-cut $\frac{18}{}$.

\subsection{Conceptual Groundwater Flow Model}

In present study, a conceptual groundwater flow model has been constructed for the Bengal Delta aquifers, Bangladesh considering all observed observations. The source and recharge processes of different types of groundwater with their chemical compositions are used to delineate the groundwater flow dynamics to represent a conceptual groundwater flow model for the Bengal Delta aquifers (Figure 10). The model takes into account the following observations: (a) basement structure and boundary condition, (b) lithology as revealed through a generalized hydrogeological cross-section, (c) surface geologic variations, and $(\mathrm{d})$ change in groundwater chemical types. Rainfall or floodwater infiltrating in to the Bengal Delta shallow aquifer through the ground, it dissolves carbon dioxide and the acidic solution $\left(\mathrm{H}_{2} \mathrm{CO}_{3}\right)$ formed reacts with carbonates in the sediments giving solutions of $\mathrm{Ca}^{2+}, \mathrm{Mg}^{2+}$ and $\mathrm{HCO}_{3}^{-}$. Silicate weathering also gives rise to $\mathrm{Ca}^{2+}$ and $\mathrm{Mg}^{2+}$ ions in solution. These hydrogeochemical reactions are responsible for the formation of
$\mathrm{Ca}-\mathrm{Mg}-\mathrm{HCO}_{3}$ type water. The initial $\mathrm{Ca}-\mathrm{Mg}-\mathrm{HCO}_{3}$ type water tends to change in to $\mathrm{Na}-\mathrm{Ca}-\mathrm{Mg}-\mathrm{HCO}_{3}$ type water due to preferentially silicate weathering, which helps to add $\mathrm{Na}^{+}$within the solution in increase with the length of the flow path. At great depths, where the residence time is long due to extremely slow flow, groundwater tends to be $\mathrm{Na}-\mathrm{Cl}$ type diluted water due to cation exchange of $\mathrm{Na}^{+}$ for $\mathrm{Ca}^{2+}$. The excess $\mathrm{Na}^{+}$comes from silicate weathering giving rise to high $\mathrm{pH}$.

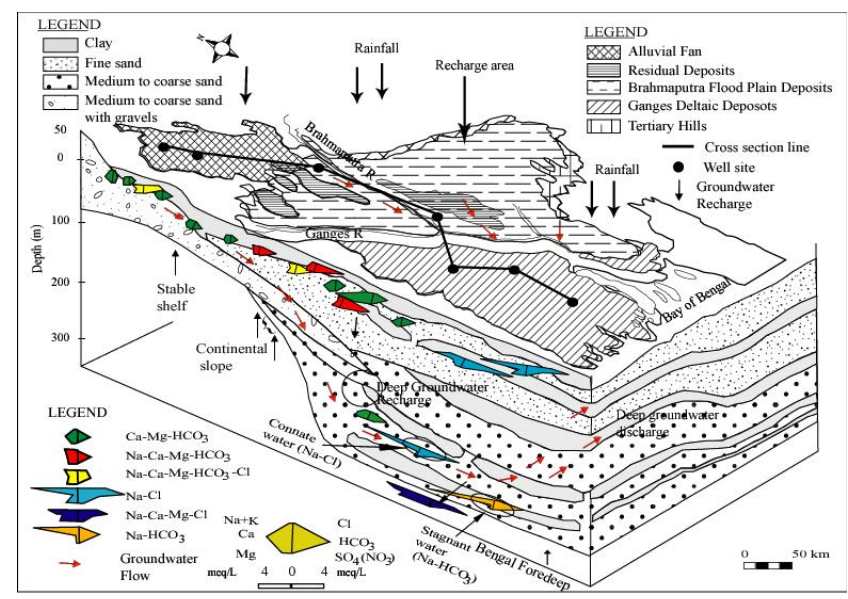

Figure 10. Conceptual groundwater flow model for Bengal delta aquifers (modified after ${ }^{12}$ ).

As per conceptual flow model (Figure 10), rainfall and/or floodwater infiltrating in the ground recharge the shallow groundwater. The shallow groundwater recharges the intermediate depth aquifers to some extend without changing its chemical facies (Figure 9b). It indicates short residence time within the intermediate depth aquifers. Thus, low water-rock interaction, which may not give rise to diluted water chemistry. Deep aquifers are recharged from the peripheral part of the study area and the recharge is possibly influenced by basement structure, surface geology and subsurface hydrogeological systems. The deep groundwater becomes chemically diluted along the flow paths to form $\mathrm{Na}-\mathrm{Cl}$ type water due to cation exchange and/or diffusion from marine clay lying within the aquifer systems. The deep groundwater aquifers of Bengal Delta are characterized by layered zones ${ }^{12}$. In Bengal Delta aquifers, as the thickness of the confining clay layers increasing down gradient (Figure 10), the deep groundwater may be squeezed out of compacting clay and the pressure of the confined water it contain increases. As a result deep groundwater discharges from confined aquifers by slow upward seepage through the overlying 
clays. Finally, deep groundwater discharges in to the Bay of Bengal as submarine groundwater discharge and this argument is supported by enriched $\delta^{18} \mathrm{O}$ values and long residence time ${ }^{12}$ as well as the upward heat flow in the coastal aquifers $\frac{33}{}$.

\section{Conclusions}

Present study has clearly demonstrated the wide spatial and depth dependence variations of the hydrochemical composition of groundwater in the Bengal Delta aquifers, Bangladesh illustrating different flow systems and aquifers. Groundwater chemistry data constrain a complex flow generally from north to south following the basement structure, topographic gradient as well the boundary conditions. By Converging all evidences based on groundwater depth of circulation, generalized hydrogeological section and hydrochemistry, three groundwater flow systems can be conceptualized with depth.

[a] A shallow flow system is observed in the west-central and southern coastal regions of the study area, where shallow groundwater shows $\mathrm{Ca}-\mathrm{Mg}-\mathrm{HCO}_{3}$ type low mineralized water.

[b] Intermediate flow system is less dominant, which acts as a transition zone between the shallow and deep aquifers and gets vertical recharge from the shallow flow system. In this system, groundwater is of less diluted due to low water-rock interactions because of short residence time.

[c] The deep groundwater flow system is enriched in chemically diluted $\mathrm{Na}-\mathrm{Cl}$ type water along the flow paths due to cation exchange and/or diffusion from marine clay lying within the aquifer systems. It possibly emerges in to the Bay of Bengal in the form of Submarine Groundwater Discharge (SGD). But the stagnant deep fresh groundwater $\left(\mathrm{Na}-\mathrm{HCO}_{3}\right.$ type) along the coastal region is completely different from the above mentioned three groundwater flow systems and it seems to be remnant of paleo-groundwater flow system persisted during past regression era.

\section{Acknowledgement}

The first author would like to acknowledge the Ministry of Education, Culture, Sports Science and Technology (MEXT), Japan for providing postgraduate scholarship during 2005-2008.

\section{References}

1. Matthess G. The properties of groundwater. department of environmental science. John Wiley and Sons Indian Nursing Council; 1982. p. 1-406.

2. Galy A, France-Lanord C. Weathering processes in the Ganges-Brahmaputra basin and the riverine alkalinity budget. Chemical Geology. 1999; 159(1-4):31-60. https:// doi.org/10.1016/S0009-2541(99)00033-9

3. Dowling CB, Poreda RJ, Basu AR. The groundwater geochemistry of the Bengal Basin: Weathering, chemsorption, and trace metal flux to the oceans. Geochimica et Cosmochimi Acta. 2003; 67(12):2117-36. https://doi. org/10.1016/S0016-7037(02)01306-6

4. Baumler R, Zech W. Soils of the high mountain region of Eastern Nepal: Classification, distribution and soil forming processes. Catena. 1994; 22(52):85-103. https://doi. org/10.1016/0341-8162(94)90019-1

5. Burbank DW. Causes of recent Himalayan uplift deduced from deposited patterns in the Ganges basin. Nature. 1992; 357:680-3. https://doi.org/10.1038/357680a0

6. Bhattacharya P, Chatteriee D, Jacks G. Occurrence of arsenic contaminated groundwater in alluvial aquifers from the Delta Plains, Eastern India: Options for safe drinking water supply. International Journal of Water Resources Development. 1997; 13(1):79-92. https://doi. org/10.1080/07900629749944

7. McArthur JM, Banerjee DM, Hudson-Edwards KA, Mishra R, Purohit R, Ravenscroft P, Cronin A, Howarth RJ, Chatterjee A, Talukder T, Lowry D, Houghton S, Chadha DK. Natural organic matter in sedimentary basins and its relation to arsenic in anoxic ground water: The example of West Bengal and its worldwide implications. Applied Geochemistry. 2004; 19(8):1255-93. https://doi.org/10.1016/j.apgeochem.2004.02.001

8. Stollenwerk KG, Breit GN, Welch AH, Yount JC, Whitney JW, Foster AL, Uddin MN, Majumder RK, Ahmed N. Arsenic attenuation by oxidized aquifer sediments in Bangladesh. Science of the Total Environment. 2007; 379(23):133-50. https://doi.org/10.1016/j.scitotenv.2006.11.029. PMid:17250876

9. Harvey CF, Swartz CH, Badruzzaman ABM, Keon-Blute $\mathrm{N}, \mathrm{Yu} \mathrm{W}$, Ali MA, et al. Arsenic mobility and groundwater extraction in Bangladesh. Science. 2002; 298:1602-6. https://doi.org/10.1126/science.1076978. PMid:12446905

10. Geological Map of Bangladesh [Internet]. [cited 1990]. Available from:

11. Majumder RK, Hasnat MA, Hossain Shahadat, Ikeuec K, Machida M. An exploration of nitrate concentrations in groundwater aquifers of central-west region of Bangladesh. Journal of Hazardous Materials. 2008; 159(2- 
3):536-43. https://doi.org/10.1016/j.jhazmat.2008.02.110. PMid:18406518

12. Majumder RK, Halim MA, Saha BB, Ikawa R, Nakamura T, Kagabu M, Shimada J. Groundwater flow system in Bengal Delta, Bangladesh revealed by environmental isotopes. Environmental Earth Sciences. 2011; 64(5):1343-52. https://doi.org/10.1007/s12665-011-0959-2

13. Kinniburgh DG, Smedley PL. Arsenic contamination of groundwater in Bangladesh. BGS Technical Report. 2001; $1: 1-21$.

14. Singh AK, Mondal GC, Kumar S, Singh TB, Tewary BK, Sinha A. Major ion chemistry, weathering processes and water quality assessment in upper catchment of Damodar River basin, Environmental Geology. 2008; 54(4):745-58. https://doi.org/10.1007/s00254-007-0860-1

15. Parkhurst DL, Appelo CAJ. Users guide to PHREEQC (version 2): A computer program for speciation, batch-reaction, one-dimensional transport, and inverse geochemical modeling. US Geol Survey Water Resources Investigations Report. 1999, pp. 1-312.

16. Allison JD, Brown DS, Novo-Gradac KJ. MINTEQA2/ PRODEFA2A geochemical assessment model for environmental systems version 3.0 user's manual. Environmental Modeling Community of Practice; 1990. p. 1-115.

17. Rahman MATMT, Majumder RK, Rahman SH, Halim MA. Sources of deep groundwater salinity in the southwestern zone of Bangladesh. Environmental Earth Sciences. 2011; 63(2):363-73. https://doi.org/10.1007/s12665-010-0707-z

18. Sikdar PK, Sarkar SS, Palchoudhury S. Geochemical evolution of groundwater in the Quaternary aquifer of Calcutta and Howrah, India. Journal Asian Earth Science. 2001; 19(5):579-94. https://doi.org/10.1016/S13679120(00)00056-0

19. Venturelli G, Boschetti T, Duchi V. Na-carbonate waters of extreme composition: Possible origin and evolution. Geochemistry Journal. 2003; 37(3):351-66. https://doi. org/10.2343/geochemj.37.351

20. Chae GT, Yun ST, Kim K, Mayer B. Hydrogeochemistry of sodium-bicarbonate type bedrock groundwater in the Pocheon spa area, South Korea: Water-rock interaction and hydrologic mixing. Journal of Hydrology. 2006; 321(14):326-43. https://doi.org/10.1016/j.jhydrol.2005.08.006

21. Ravenscroft P, McArthur JM. Mechanism of regional enrichment of groundwater by boron: The examples of Bangladesh and Michigan, USA. Applied Geochemistry. 2004; 19(9):1413-30. https://doi.org/10.1016/j.apgeochem.2003.10.014
22. Drever JI. The geochemistry of natural waters. Prentice Hall, Englewood Cliffs; 1988. p. 1-5.

23. Breit GN, Yount JC, Uddin MN, Muneem AA, Lowers HA, Berry CJ, Whitney JW. Compositional data for Bengal Delta sediment collected from a borehole at Rajoir, Bangladesh. United States Geological Survey; 2007. p. 1-46.

24. Gaillardet J, Dupre B, Louvat P, Allegre CJ. Chemical Geology. 1999; 159(1-4):3-30. https://doi.org/10.1016/ S0009-2541(99)00031-5

25. Taylor SR, McLennan SM. The continental crust: Its composition and evolution. Geological Journal. 1986; 21(1):85-6.

26. Li X, Zhang L, Hou X. Use of hydrogeochemistry and environmental isotopes for evaluation of groundwater in Qingshuihe Basin, Northwestern China. Hydrogeology Journal. 2008; 16(2):335-48. https://doi.org/10.1007/ s10040-007-0269-7

27. Appelo CAJ, Postma D. Geochemistry, groundwater and pollution. Balkema, Rotterdam, The Netherlands; 1993. p. 1-647.

28. Carrillo-Rivera JJ, Varsányi I, Kovács LÓ, Cardona A. Tracing groundwater flow systems with hydrogeochemistry in contrasting geological environments. Water Air and Soil Pollution. 2007; 184(1):77-103. https://doi.org/10.1007/ s11270-007-9400-6

29. Clark ID, Fritz P. Environmental isotopes in hydrogeology. CRC Press; 1997. p. 1-352.

30. Cloutier V, Lefebvre R, Savard MM, Bourque E, Therrien R. Hydrogeochemistry and groundwater origin of the BassesLaurentides sedimentary rock aquifer system, St. Lawrence Lowlands, Quebec, Canada. Hydrogeology Journal. 2006; 14(4):573-90. https://doi.org/10.1007/s10040-005-0002-3

31. Mukherjee A, Fryar AE. Deeper groundwater chemistry and geochemical modeling of the arsenic affected western Bengal basin, West Bengal, India. Applied Geochemistry. 2008; 23(4):863-94. https://doi.org/10.1016/j.apgeochem.2007.07.011

32. DPHE (Department of Public Health Organization) (2006) Final report on development of deep aquifer database and preliminary deep aquifer map (First Phase), Department of Public Health Engineering, Local Government Division, Ministry of LGRD and Co-operatives, Government of the People's Republic of Bangladesh

33. Majumder RK, Shimada J, Taniguchi M. Groundwater flow systems in the Bengal Delta, Bangladesh, inferred from subsurface temperature readings. Songklanakarin Journal of Science and Technology. 2013; 35(1):99-106. 